\title{
Optical Parametric Devices
}

\author{
by
}

\author{
Meng Lon Iu
}

A thesis submitted in conformity with the requirements for the degree of Master of Applied Science

Graduate Department of The Edward S. Rogers Sr. Department of Electrical and Computer Engineering University of Toronto

(c) Copyright 2016 by Meng Lon Iu 


\begin{abstract}
Optical Parametric Devices

Meng Lon Iu

Master of Applied Science

Graduate Department of The Edward S. Rogers Sr. Department of Electrical and Computer Engineering

University of Toronto

2016

In this thesis, a continuous range of NIR generation from $1650 \mathrm{~nm}$ to $2160 \mathrm{~nm}$, for the first time, has been experimentally demonstrated in a monolithic BRW semiconductor platform when pumped at non-degenerate wavelength. When pumped at degenerate wavelength, the wavelength conversion in BRW has experimentally shown a 3dB bandwidth of $100 \mathrm{~nm}$ with $-37 \mathrm{~dB}$ conversion efficiency. The potential of optical parametric amplification in BRW is studied through NLSE solver and experimentally characterized. BRW diode laser, which is designed for self-pump process with pump power at least an order higher than external pump can achieve, is characterized for the laser and nonlinear performance and is shown to work at 780nm regime. Experimental results and simulation model suggests that self-pumping process is highly feasible and can operate at room temperature. To alleviate the difficulties self-pumping imposed on the phasematching, acceptance bandwidth enhancement from $0.5 \mathrm{~nm}$ to $17 \mathrm{~nm}$ through tapering is experimentally demonstrated.
\end{abstract}


To my significant other, Yuki Nishimura.

Without your affection, love and encouragement, I would not be who I am, see the world with so much vibrant color, and be proud of myself.

I dedicate this thesis to you. 


\section{Acknowledgements}

First, I would like to thank my supervisor, Professor Amr Helmy. His patience, tolerance, motivation, and support has guided me through these two years of academic development. His charm and words of wisdom have assisted me through difficult times and his expense has surely increased due to my influence in purchasing various items. This thesis would not be possible with his guidance and support.

I would also like to thank my Thesis Committee, Professor Ted Sargent, Professor Nazir Kherani, and Professor Peter Lehn at University of Toronto, who have taken some of their precious time and agreed serving on the committee in such a late request. I greatly appreciate your time, kind words and your valuable comments.

I am particularly grateful to Dr. Nima Zarein and Dr. Dongpeng Kang. They patiently taught me my experimental characterization skills and helped me through many of my misunderstanding of the theory in the research. I would also like to thank Dr. Osman Ahmed and Dr. ZhiZhong Yan for their expertise in research and knowledge that they have shared with me, Ryan Marchildon for teaching me about Quantum theory, PoHan Chang for the plasmonic theory that he will one day teach me, Rajiv Prinja for all the fabrication that he has done, Eric Chen for all the fabrication that he has done and will continually do, Yiwen Su for letting me take apart his setup again and again, Haoyu He for teaching me many things that I did not know about the characterization for the quantum theory, Grace Zhong for continually taking away my snacks in the drawer, Charles Lin, Herman Wong, and Steve Rutledge who all have helped me with miscellaneous things. To Basil Eleftheriades, whose last name I still cannot get it right, and also Gleb Egorov, whose last name I would never gotten it right, they have most certainly make this a much smoother journey. Thank you for all the people that have assisted me during my Master.

To my brother, Jonathan, thank you for helping me during my years in Canada and for the many years to come. I own my deepest gratitude to my mother who has supported 
me throughout all my life. Without her guidance and teaching, I would not have come this far, appreciate my life to this profound degree, and achieve so much. I wish her the best of luck for her completion of her third Master Degree in this year. 


\section{Contents}

1 Introduction $\quad 1$

1.1 Applications in mid-IR frequency conversion . . . . . . . . . . . . 2

1.1.1 Mid-IR Laser sources . . . . . . . . . . . . . . . . . . . . . 3

1.2 Applications in Optical Networks . . . . . . . . . . . . . . . . 6

1.2.1 Optical amplifiers . . . . . . . . . . . . . . 6

1.2.2 Wavelength conversion technology and applications . . . . . . . 8

1.3 Existing limitations of current $\chi^{2}$ and $\chi^{3}$ Medium as optical parametric devices . . . . . . . . . . . . . . . . . . . . 10

1.3.1 Types of Optical Parametric systems . . . . . . . . . . . . 10

1.4 Motivation . . . . . . . . . . . . . . . . . . 13

1.5 Thesis Overview . . . . . . . . . . . . . . . . . . . . 15

2 Background $\quad 16$

2.1 Nonlinear Optical Parametric processes and its applications . . . . . 16

2.1.1 Phasematching Techniques . . . . . . . . . . . . . . 20

2.1.2 Difference frequency Generation Mathematical representation . 21

2.1.3 Difference Frequency Generation Applications . . . . . . . . . . . 23

2.2 Bragg Reflection Waveguide as a monolithic nonlinear platform . . . . . 24

2.2.1 Bragg Reflection Waveguide Structural details . . . . . . . . . . . 24

2.2.2 Bragg Reflection Waveguide as a nonlinear medium . . . . . . . 25 
2.3 Bragg Reflection Waveguide Diode Laser . . . . . . . . . . . . . . 29

2.4 NLSE Solver . . . . . . . . . . . . . . . . . . . . . . . . . 32

2.4.1 Mathematical formulation . . . . . . . . . . . . . 32

3 Bandwidth of optical parametric devices 35

3.1 Bandwidth Enhancement through tapering . . . . . . . . . . . . 36

3.2 Characterization of the Tapered Waveguides . . . . . . . . . . . . 42

3.2.1 Pump bandwidth Characterization: Second Harmonic Generation 42

3.2.2 Pump bandwidth Characterization: Difference Frequency Generation 46

3.2 .3 Signal Bandwidth Characterization . . . . . . . . . . . . 48

3.3 Summary of the BRW bandwidth and its enhanced bandwidth design . . 50

4 Parametric Generation in BRW structure $\quad 52$

4.1 Optical Parametric Gain . . . . . . . . . . . . . . . . . . . . 52

4.1 .1 NLSE simulation . . . . . . . . . . . . . . 53

4.1 .2 Continuous wave characterization $\ldots \ldots \ldots \ldots . \ldots 57$

4.1 .3 Pulse-CW characterization . . . . . . . . . . . . . . . . 60

4.1.4 Wavelength Conversion . . . . . . . . . . . . . . . 63

4.2 Summary of the parametric generation of the optical parametric devices . 65

5 Parametric Generation in BRL $\quad 67$

5.1 Bragg Reflection Waveguide Laser Diode Design . . . . . . . . . . . 68

5.2 BRL Laser Performance . . . . . . . . . . . . . . . . . . 70

5.2 .1 Temperature Effect $\ldots \ldots \ldots \ldots \ldots \ldots \ldots$

5.2 .2 Current Effect . . . . . . . . . . . . . . . . 74

5.3 BRL Nonlinear performance . . . . . . . . . . . . . . . . 75

5.3 .1 Temperature effect . . . . . . . . . . . . . . 77

5.3 .2 Current Effect . . . . . . . . . . . . . . . . . . . 79

5.4 Self-pumped Experiment . . . . . . . . . . . . . . . . . . 82 
5.5 Summary of Parametric generation in BRL . . . . . . . . . . . . . . . . . 84

6 Conclusion and Future Outlook $\quad 85$

6.1 Future Work . . . . . . . . . . . . . . . . . . . . . . . . 88

$\begin{array}{ll}\text { Bibliography } & 89\end{array}$ 


\section{List of Tables}

3.1 Structural Information of the Tapered Waveguides _. . . . . . . . . 40

3.2 Different Types of SHG . . . . . . . . . . . . . . . . 43

4.1 Waveguide Parameters Used in NLSE Solver . . . . . . . . . . . 54 


\section{List of Figures}

1.1 Simulated absorption bands of various molecules in the $3 \mu \mathrm{m}$ to $5 \mu \mathrm{m}$ spectrum. From $[11] \ldots \ldots \ldots \ldots \ldots \ldots \ldots$

2.1 Symbolic Diagram for Difference Frequency Generation (DFG). Two sources, the pump and the signal, are injected into the $\chi^{2}$ medium to perform DFG. An addition wavelength, the idler, is generated. . . . . . . . 17

2.2 Tuning Curve of the BRW Structure. . . . . . . . . . . . . . . 19

2.3 Simulated Results based on the solution from Equation . . . . . . . . . 23

2.4 Bragg Reflection Waveguide Modal Structure. Top Left: Effective index of the fundamental mode. Top Right: Effective index of the Bragg mode.

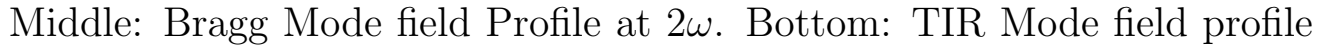

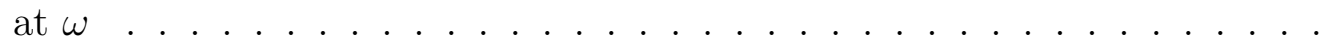

2.5 2D Mode Profile of the Type 2 DFG. Left is the Bragg mode with wavelength at $775 \mathrm{~nm}$ guided by the structure. Center is the TE TIR Mode with wavelength $1550 \mathrm{~nm}$. Right is the TM TIR Mode with wavelength at

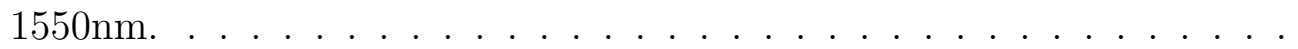

2.6 1D BRW Modal Analysis. Top Left: Effective index of the TE Bragg Mode at $2 \omega$. Top Middle: Effective Index of TE TIR Mode at $\omega$. Top Right: Effective index of the TM TIR Mode at $\omega$. Bottom: Modal profiles of each modes. . . . . . . . . . . . . . . . 
3.1 Simulated normalized SHG Spectrum, with permission to reproduce from $[66]$

3.2 2D Cross section of Bragg Reflection Waveguide Structure. A full description of the structure can be found in $[62] \ldots \ldots \ldots \ldots$

3.3 Fabrication Flow regarding the tapering implementation on the AlGaAs

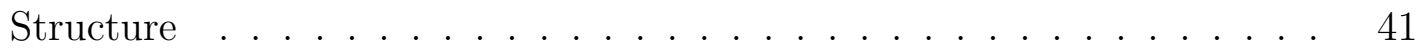

3.4 SHG Characterization Setup for CW Input . . . . . . . . . . . . . . . . 43

3.5 These figures show the measured data resulted from the SHG characterization. The bottom x-axis shows the generated second harmonics wavelength while the top $\mathrm{x}$-axis shows in the injected C-Band pump wavelength. The y-axis shows the linearly normalized measured power of the SHG. (a)-(f) corresponds to the Structure A-F shown in Table 3.1 . . . . . . . . . . . 45

3.6 DFG Characterization Setup for CW Input . . . . . . . . . . . . . . . . . 47

3.7 These figures show the generation of idler in power and wavelength in response to detuning of the pump. The bandwidth range is increased from

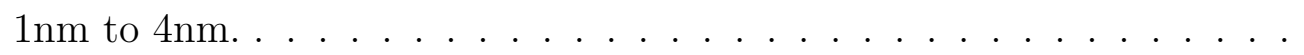

3.8 Estimated Bandwidth from the measured data of second harmonic generation and Idler generation . . . . . . . . . . . . . . .

3.9 (a) Output spectrum of the DFG with a straight waveguide. (b) Wavelength Conversion showing a peak conversion efficiency of $-37 \mathrm{~dB}$ and $100 \mathrm{~nm}$ FWHM bandwidth. . . . . . . . . . . . . . . .

4.1 The figures above shows the nonlinear behavior of the continuous wave inside the waveguide at (a) $40 \mu \mathrm{m}$, (b) $500 \mu \mathrm{m}$ and (c) $1000 \mu \mathrm{m}$. . . . . . .

4.2 (a) The simulated nonlinear interaction as a function of propagation within the Bragg Reflection Waveguide (BRW) with $1 \mathrm{~mW}$ pump, $0.1 \mu \mathrm{W}$ Signal. (b) Simulated Gain of the device as a function of input pump power. (c) Simulated Idler Conversion efficiency as a function of input pump power. 
4.3 The figures above shows the nonlinear behavior of the continuous wave inside the waveguide at (a) $40 \mu \mathrm{m}$, (b) $500 \mu \mathrm{m}$ and (c) $1000 \mu \mathrm{m} \ldots \ldots . . . .56$

4.4 The figures above shows the nonlinear behavior of the continuous wave inside the waveguide at (a)40 $\mu \mathrm{m}$, (b) $500 \mu \mathrm{m}$, (c) $1000 \mu \mathrm{m}$ and (d) $2000 \mu \mathrm{m}$.

4.5 The output spectrum of the DFG process. Green is when the pump is off and blue is when the pump is on. The conversion efficiency is $-36.9 \mathrm{~dB}$. .

4.6 Output signal power with the pump on (Blue) and the pump off(Green) at different wavelength. Notice the higher order Fabry Perot effects. Unable to isolate the gain of the signal. . . . . . . . . . . . . . .

4.7 Experimental Setup for DFG with the Femtosecond Ti:Sapphire laser as the pump source and the Continuous Wave (CW) Tunable C-Band laser as the signal source. . . . . . . . . . . . . . . .

4.8 Measured Signal power with different Femtosecond pump input power at different wavelength. The data indicates the Fabry Perot Effect. . . . . .

4.9 Power measurement of the signal power with fast photodiode. The Electrical system is triggered by the sync signal of the Femtosecond laser. The sharp rise and the gradual drop of the signal power is in agreement with the pulse-CW simulation from the NLSE Model . . . . . . . . . . 63

4.10 NIR Generation Experimental setup with CW Pump and Pulse Signal . . 64

4.11 Normalized Fitted NIR Generation Data, demonstrating the capability to operate outside of the degenerate regime and perform $\mu \mathrm{m}$ wavelength conversion. . . . . . . . . . . . . . . . .

5.1 (a) The 1D Mode solver result showing the effective indices of each waves as well. It also shows the field profiles of the pump Bragg TE mode (Red), pump TIR TE Mode (Black), Fundamental TIR TE Mode(White) and Fundamental TIR TM modes. (b) shows the details of the AlGaAs layers for Bragg Reflection Waveguide Diode laser (BRL) 8 Structure. . . . . . 
5.2 (a) BRL 8 Waveguide after growing of electrical insulation silica layer. (b) BRL 8 Wavelength after $\mathrm{Ti}$ and Au contact deposition. . . . . . . . 70

5.3 Experimental Setup for characterizing laser performance of the laser diode sample. . . . . . . . . . . . . . . . . . . .

5.4 (a) LIV of a bonded Bragg laser diode. Threshold current is $44 \mathrm{~mA}$ and threshold voltage is $2.4 \mathrm{~V}$. (b) $\ldots \ldots \ldots \ldots \ldots \ldots$

5.5 (a) BRL lasing spectra from $15^{\circ} \mathrm{C}$ to $85^{\circ} \mathrm{C}$. (b) Extracted linear relationship between the lasing wavelength and the change in temperature. A shift of lasing temperature at around $0.197 \mathrm{~nm} /{ }^{0} \mathrm{C} \ldots \ldots \ldots$

5.6 (a) Unbonded $2 \mu \mathrm{m}$ ridge Laser Output spectra at different current level with pulse width at $1 \mu \mathrm{s}$ and pulse delay at $20 \mu \mathrm{s}$.(b) Bonded $2.2 \mu \mathrm{m}$ ridge Laser spectra output at pulse mode and at DC mode. (c) Extracted unbonded $2 \mu \mathrm{m}$ ridge laser output wavelength with respect to DC current

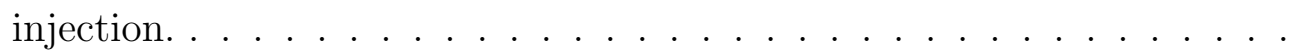

5.7 Experimental Setup for SHG nonlinear performance characterization with laser diode. CW laser source is used to characterize the laser diode nonlinear conversion efficiency optically. Output of the second harmonic is collected with the Si detector. Femtosecond Laser source is used to characterize the phasematching behavior in response to temperature and current effects. SH output is collected into fiber and guided into an OSA for

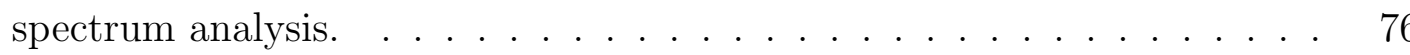

5.8 SHG Spectrum generated from CW tunable source . . . . . . . . . . 77

5.9 (a) Optically pumped SHG Spectra of the laser diode sample at different temperature. (b) The linear fit and the trend of the shift in phasematching wavelength in response to different temperature. . . . . . . . . . . 
5.10 (a) Measured Spectra of the output from the laser diode sample injected with $50 \mathrm{~mW}$ Femtosecond pulse at different current. (b) A different representation to indicate the shift of the phasematching wavelength and the lasing wavelength. (c) The net shift of the phasematching wavelength for Type-1, Type-0 and Type-2. (d) The output power of each type of SHG . Power of Type-1 and Type-0 SHG remains relatively constant. The is a apparent gain behavior for the Type-2 SHG. Indicating the Bragg mode generated from the SHG is being amplified within the electrically active

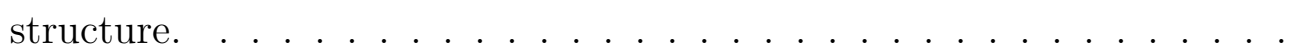

5.11 Exponential fit for the carrier effect uncoupled the thermal effect from the current injection. Strong indication of the contribution of the blue shift from the free carriers . . . . . . . . . . . . . . .

5.12 (a) Laser and phasematching wavelength from the Eq. 5.7 with lasing wavelength as $784 \mathrm{~nm}$ and $\frac{\Delta T}{\Delta I}$ as $0.3^{\circ} \mathrm{C} / \mathrm{mA}$. The intersection indicates the position where the self-pumping will occur (b) The line indicate the position where the lasing wavelength and the phasematching position coincides. The wavelength indicated at the legend is assumed to be the lasing wavelength at $30^{\circ} \mathrm{C}$ with $60 \mathrm{~mA}$ direct current. . . . . . . . . . 


\section{Acronyms}

2PA Two-photon Absorption. 12

BPM Birefringent Phasematching. 24

BRL Bragg Reflection Waveguide Diode laser. xiii, 14, 68-71, 74, 76, 85

BRW Bragg Reflection Waveguide. xii, 14, 15, 24, 25, 32, 53, 56, 60, 64, 66, 68, 69

CW Continuous Wave. xii, 55-58, 61, 62, 65, 66

DFB Distributed Feedback Laser. 3, 4

DFG Difference Frequency Generation. x, xii, 1, 4, 5, 9, 14, 17, 18, 21-23, 53, 60-62, 66,67

DWDM Dense Wavelength Division Multiplexing. 8

EDFA Erbium Doped Fiber Amplifier. 6, 7, 11

FWM Four Wave Mixing. 1, 12

HNLF Highly Nonlinear Fiber. 5

ICL Interband Cascade Laser. 4, 5

MOCVD Metall Organic Chemical Vapor Deposition. 24 
NF Noise Figure. 11

NLSE Nonlinear Schrolinger Equation Solver. 32

OPO Optical Parametric Oscillator. 10

PCF Photonic Crystal Fiber. 11

PPLN Periodically Poled Lithium Niobate. 12, 20

QAM Quadrature Amplitude Modulations. 9, 12

QCL Quantum Cascade Laser. 3-5

QPM Quasi-Phasematching. 10, 12, 20, 24

SEM Scanning Electron Microscope. 71

SFG Sum Frequency Generation. 1

SHG Second Harmonics Generation. 1

SOA Semiconductor Optical Amplifier. 6, 7

SOI Silicon on Insulator. 12

SPM Self-Phase Modulation. 12

SRA Stimulated Raman Amplifier. 6, 7

TIR Total Internal Reflection. 25

WDM Wavelength Division Multiplexing. 8, 9

XGM Cross-Gain Modulation. 9

XPM Cross-Phase Modulation. 9, 12 


\section{Chapter 1}

\section{Introduction}

Second Order nonlinear optical susceptibility $\chi^{2}$ has a wide span of applications. It plays an important role in optical telecommunication[1][2], quantum information[3], and spectroscopy [4][5]. Frequency mixing provided by the optical nonlinearity is utilized to open more possibilities where linear optics cannot provide. In nonlinear optics, frequency mixing is possible through the parametric energy level of the system[6]. In $\chi^{2}$ Medium, the following frequency mixing are allowed : DFG, Second Harmonics Generation (SHG), and Sum Frequency Generation (SFG). In particular, difference frequency generation has the most profound usage in optical telecommunication[7] and mid-IR Spectroscopy[8][9]. $\chi^{3}$ optical nonlinearity also allows frequency mixing process called Four Wave Mixing (FWM) [6]. However, it has a much lower nonlinearity and smaller bandwidth of interaction provided the same pump power. Therefore, such limitations in the strength of nonlinearity and bandwidth would restrain $\chi^{3}$ applications to require a higher power injection or longer interaction length to compensate for the lower nonlinearity. It also has a less flexible wavelength of operation due to the smaller bandwidth given a fixed pump wavelength. As a result, $\chi^{3}$ is less favourable compared to $\chi^{2}$ for certain applications targeting in low power, miniaturized, integrated design. 


\subsection{Applications in mid-IR frequency conversion}

By using mid-infrared (mid-IR) spectrum, identification of many gaseous molecules are possible[10]. Molecules have their characteristic rotational-vibrational bands in the midIR spectrum. Correspondingly, they have their own specific absorption spectra in the mid-IR spectrum due to their energy band, shown in Figure 1.1. Therefore, by analyzing the absorption spectra in such region, the type of molecules and its corresponding concentration can be uniquely identified. In particular, the mid-IR spectrum has two atmospheric transmission windows, which are in $3 \mu \mathrm{m}-5 \mu \mathrm{m}$ and $8 \mu \mathrm{m}-14 \mu \mathrm{m}$. These windows are crucially important for atmospheric applications as they allow low absorption for those two windows in spectra in the atmosphere. Therefore, this region has garnered tremendous technological interest as it allows many applications such as mid-IR laser absorption spectroscopy[11], trace gas sensing[12], and remote sensing[13]. For high accuracy spectroscopic identification of gaseous molecules, laser sources for this mid-IR spectrum are particularly important. As lasers are capable of high intensity and narrow linewidth generation, it allows high spectroscopic selectivity and sensitivity. With the use of widely tunable laser system and various signal enhancement techniques such as cavity enhanced spectroscopy, a detection limit of parts-per-trillion has been demonstrated in a table top setup[14]. Such deployment is extremely useful for experimental or stationary

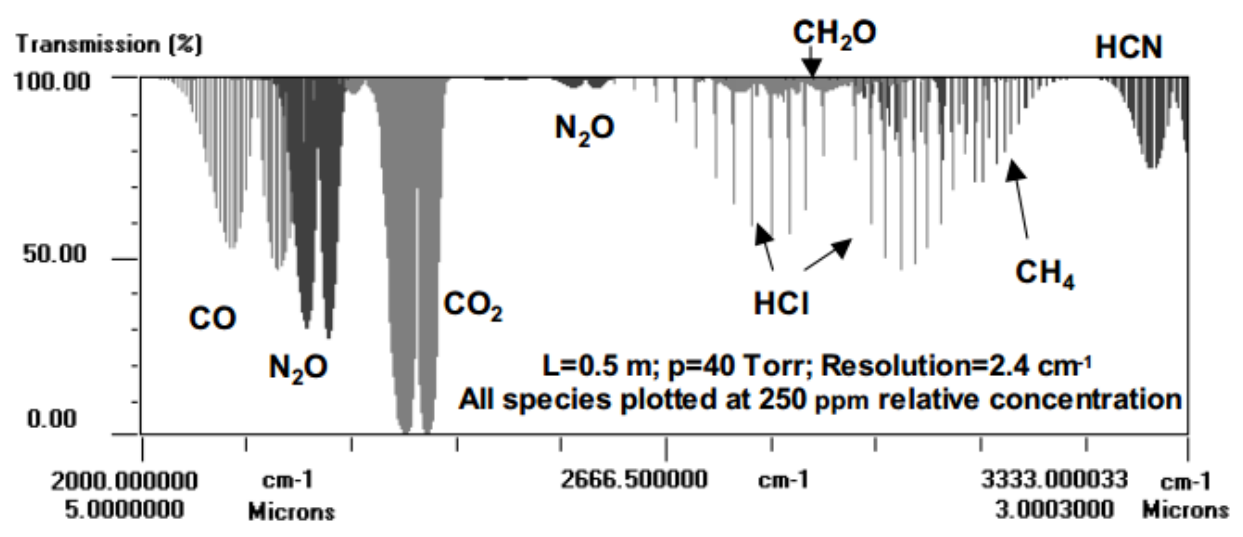

Figure 1.1: Simulated absorption bands of various molecules in the $3 \mu \mathrm{m}$ to $5 \mu \mathrm{m}$ spectrum. From [11] 
application. But for portable, lower power applications, this extremely high sensitivity, yet immobile deployment is not feasible. To achieve the goal of a robust, compact, portable, low cost and high sensitivity application outside laboratory or stationary environment, the laser system must be integrated on-chip and has a high efficiency for such application.

\subsubsection{Mid-IR Laser sources}

There are three major types of laser sources capable of generating mid-IR wavelength. They are Quantum Cascade Lasers, Antimonide lasers and nonlinear frequency conversion based sources.

\section{Quantum Cascade Laser}

Quantum Cascade Laser (QCL) is a semiconductor injection laser[15]. It is different from normal diode lasers, where the photons are generated through the radiative transition between the conduction band and the valence band. QCL is utilizing its radiative inter-subband transitions to generate the mid-IR wavelength[16]. In another words, the generation is based solely on the electronic transitions between the sub-bands within the conduction band. These sub-bands transition are made possible through engineering with the thickness of periodic quantum well and quantum barrier that arises from the layer quantization in the semiconductor heterostructure. In recent year, QCL designs have achieved a wavelength range in the mid-IR from $3 \mathrm{um}$ to $25 \mu \mathrm{m}$ Yet, it has an limited temperature tunability of $40 \mathrm{~nm}$ around $5 \mu \mathrm{m}[17]$. It has been demonstrated a total wavelength tunability of $200 \mathrm{~nm}$ at around $4.5 \mu \mathrm{m}$ with $150 \mathrm{~mW}$ from an array of 32 Distributed Feedback Laser (DFB) QCLs[18]. An even higher tunability can be achieved if an external cavity design is utilized[17]. QCL has also been shown for monolithic integration with detectors[19]. However, such design suffers from multiple issues, namely resulting from high forward voltage, high threshold current density[20]. Such issues is resulting 
from the high number of epitaxial layers in the devices. During a continuous wave operation of QCL, a large amount of heat is generated due to its high forward voltage and high threshold current density. This intrinsic property of QCL results in low wall plug efficiency[21] for proper operation in room temperature.

\section{Antimonide Laser}

Antimonide lasers[22], similar to conventional diode laser, utilizes the radiative interband transition between the conduction band and the valence band. It is possible to generate the mid-IR wavelength because antimonide family has a small band gap[23]. However, also due to the small band gap, it is very susceptible to Auger recombination, resulting in low radiative efficiency at room temperature. In order to mitigate the Auger recombination effect, interband cascade laser based on antimonide has been demonstrated and have shown to significantly suppress the Auger recombination[24]. However, it has similar efficiency issues as QCL due to the high number of periodic structures for the quantum well and quantum barriers. Interband Cascade Laser (ICL) has been shown to work in cw operation around 3-6 $\mu \mathrm{m}[25]$. It can have a low threshold current density, about 134 $\mathrm{A} \mathrm{cm}^{-2}$ at $3.6 \mu \mathrm{m}$ at $300 \mathrm{~K}[26]$. With a DFB structure design, a $14 \mathrm{~mW}$ single mode $\mathrm{cw}$ operation at $20 \mathrm{C}^{0}$ has been demonstrated around 3.7-3.8 $\mu \mathrm{m}[27]$. At room temperature, its tunability is only about $40 \mathrm{~nm}$ around $3.6 \mu \mathrm{m}$; but at $80 \mathrm{~K}$, an electrical tunability of $600 \mathrm{~nm}$ around $4.5 \mu \mathrm{m}$ has been demonstrated[28].

\section{Mid-IR Sources based on Nonlinear Frequency Conversion}

Nonlinear frequency generation can be utilized to generate mid-IR frequency. DFG has shown to down-convert light from near-IR to mid-IR wavelength with a wide tunability[29]. Such wide conversion would require the use of DFG in $\chi^{2}$ Process[6]. To facilitate a nonlinear frequency conversion, the pump, signal, and idler that are interacting will need to satisfy the phasematching condition and energy conservation in the $\chi^{2}$ nonlinear medium. 
Common $\chi^{2}$ medium which are commonly used are crystals such as lithium niobate $\left(\mathrm{LiNbO}^{3}\right)$, potassium titanate phosphate $(\mathrm{KTP})$, and barium borate (BBO). However, they are not suitable for monolithic integration. As for semiconductor based material, Silicon nano-waveguides, which utilizes $\chi^{3}$ DFG, has demonstrated to function as a nonlinear passive medium for wavelength conversion at $2.4 \mu \mathrm{m}[30]$. However, it requires an additional stage of DFG through a Highly Nonlinear Fiber (HNLF) to generate the require pump and signal light beams into the waveguide. This solution is not favourable as the lack of monolithic active design in silicon photonics[31]. GaAs semiconductor platform has a strong $\chi^{2}$ susceptibility. Active and passive designs are readily available. Yet, its lack of birefringence has proven the phasematching condition to be difficult and would require periodic poling to achieve phasematching.

\section{Summary of mid-IR sources}

Widely tunable mid-IR integrated device operating at room temperature is currently lacking. QCL and ICL are capable of generating a wide spectrum of wavelength in the mid-IR regime. In particular, ICL has the most promising parameters as it has a low threshold voltage and low threshold current density. Yet, these lasers lack in ease of tunability. Their temperature tunability can only achieve at the order of tens of nanometers. Indeed, wider tunability can be achieved, as stated in the previous sections, through the use of external cavity. It can also be achieved with an array of lasers lasing in a range of different frequencies to have a wide tunability as a whole. But such solution is not monolithic or results in a costly structure. Mid-IR sources based on wavelength conversion can easily achieve the level of tunability, for which the other two candidates cannot without an external cavity. Therefore, this is an interest in utilizing semiconductor material as a nonlinear platform to achieve a strong and wide bandwidth wavelength conversion integrated converter. 


\subsection{Applications in Optical Networks}

Telecommunication is the act of transmitting data over a distance through a channel. It allows us to form an information network that enable our need of high-speed, high bandwidth data usage. One of the most important telecommunication network is optical fiber network[32]. It is extremely useful and powerful compared to the use of electrical signal in copper wire due to its lack of cross-talk behavior, frequency-bandwidth electrical characteristic limitation, and low loss compared to the electrical network. It is excellent for high bandwidth, long distance transmission purposes.

To support optical network for an extra long distance transmission, there is a need for an optical amplifier for increasing the signal power along the transmission link in order to mitigate the loss induced by such long distance transmission. $\chi^{2}$ can play an important role in this area as the parametric nonlinear process allows the signal in the 1550nm range to be amplified through the use of a pump in the $775 \mathrm{~nm}$. Other than amplification, the capability of the parametric device to perform wavelength conversion[33] is also crucial as wavelength conversion can facilitate and improve the interconnections in all-optical network[1] as well. Therefore, optical parametric device can be a single platform for multiple important applications in the Optical Communication.

\subsubsection{Optical amplifiers}

Conventional optical amplifiers used in optical network are Erbium Doped Fiber Amplifier (EDFA)[34], Semiconductor Optical Amplifier (SOA) [35], and Stimulated Raman Amplifier (SRA) [36]. EDFA and SRA are fiber-based where it requires length at the order of meters to kilometers depending on the structure to get a strong gain up to 50dB, while SOA a semiconductor chip with a lesser gain up to $30 \mathrm{~dB}$. However, SOAs can be monolithically fabricated with the nano-fabrication technology. It can be easily scaled up with mass production. At first glance, SOA may seems superior in size and in fab- 
rication. It can be easily cascaded with more SOAs along the link to achieve a higher gain and transmit signal further than the fiber counterpart. However, optical amplifier does not simply require to be high in power gain of the signal, but also require to be low in noise introduction. It is the signal degradation from the intrinsic noise introduced by the amplifier that is imposing a maximum distance for the optical signal to travel. The added noise from the amplifier can degrade the signal to noise ratio (SNR). As more noise is added and the SNR drops below a threshold, the information embedded within the signal can no longer be extracted. Therefore, SOA, which in general has a higher noise figure than EDFA or SRA, is undesirable for a long transmission link. Opto-electrical converter is also available to recover the signal to noise ratio. It can decipher the optical signal to electrical signal, and then encode the signal back into optical signal. In this way, the signal can be regenerate completely before it is too weak or too noisy to be decode. However, opto-electrical regeneration requires a specific design for a specific modulation scheme and bit rate. It is not generally applicable to all schemes and bit rate. Moreover, the high number of multiplexers and demultiplexers required for a high-bandwidth network to achieve opto-electrical regeneration would be too expensive and undesirable to be implemented.

On the other hand, Optical Signal can also be amplified with the optical parametric process[37]. Instead of relying on the above-stated amplifiers which are based on real energy state interaction, optical parametric amplification relies on virtual energy state interaction. This implies that the amplification process is less subjective to noise. It has been demonstrated with fiber based system to have a lower noise figure that any of the stated amplifier[38] with a wide band gain to $80 \mathrm{~nm}$. The lowest measured fiber based optical parametric amplifier has a noise figure of $3.7 \mathrm{~dB}[39]$. This measured noise figure is larger than the $3 \mathrm{~dB}$ quantum limit imposed on all optical amplifier. The difference between the measured and the theory is experimentally investigated to be relating to Raman effects[40], since the pump is very close the signal for $\chi^{3}$ optical parametric 
amplifier. If there is a $\chi^{2}$ based amplifier, noise figure can likely to be reduced even more. Therefore, it is highly motivated to make this type of amplifier widely accessible.

\subsubsection{Wavelength conversion technology and applications}

In optical communication, Dense Wavelength Division Multiplexing (DWDM) allows a large capacity of information to be transmitted, as many channels are utilized at the same time[41]. To facilitate the utilization of this scheme, wavelength-routed network is implemented to manage and improve the utilization efficiency[42]. One of the key aspect of this implementation is the requirement of wavelength conversion[1]. Wavelength Conversion allows the conversion of wavelength to a different wavelength. It is a necessity to interface different networks, to switch and route within the network, and to bridge future network development when the wavelength channels are utilized different across various network architectures. This can solve the interoperability, scalability, and transparency issues of the DWDM in wavelength-routed network.

Wavelength Conversion can be achieved with eletro-optic converter where a signal in wavelength $\lambda_{1}$ is demultiplexed, detected and transformed to electrical domain, and regenerated in wavelength $\lambda_{2}$. However, such conversion is expensive and has a large power consumption as it consists of many high-end electrical components to achieve the speed and the capability of handling many channels simultaneously As a result, this will form the major bottleneck of the optical network.

To alleviate the electrical bottleneck, all optical network is desired. All optical network is a network where there is no optoelectronic regeneration. This will satisfy the optical transparency in the Wavelength Division Multiplexing (WDM) scheme. As a result, optical phenomena is needed to achieve wavelength conversion. For the wavelength conversion to integrate with the all-optical network seamlessly, the wavelength conversion technique should ideally be polarization insensitive, highly efficient, and simple to implement. Some of the earlier techniques are cross-gain modulation and cross-phase 
modulation in semiconductor optical amplifier[43]. However, they can only applied to amplitude-modulated on-off key scheme and does not support any phase related scheme, such as the popular Quadrature Amplitude Modulations (QAM). They also have a bitrate limitation determined by the carrier dynamics as the both Cross-Gain Modulation (XGM) and Cross-Phase Modulation (XPM) relies on inter-band recombination.

In order to achieve wavelength conversion while preserving the phase and amplitude information for the QAM with fast response time, optical nonlinear frequency mixing can be exploited to achieve the wavelength conversion. Nonlinear optical frequency mixing is an ultrafast process. It has a bit-rate transparency as it does not relies on carrier dynamics. It also has transparency in modulation scheme, as it is sensitive to both amplitude and phase information[44]. Four wave mixing in $\chi^{3}$ [45] and DFG in $\chi^{2}$ [46] have both been demonstrated to achieve wavelength conversion. They can allow multiple channels to be convert simultaneously. In comparison, the two difference types of nonlinearity $\chi^{3}$ system have its own drawback. In $\chi^{3}$, a pump source at $\omega_{p}$ and the signal $\omega_{s}$ are injected into a nonlinear medium as a highly nonlinear medium such as SOA [45] or [47]. A new converted wavelength $\omega_{i 1}$ will be generated at $2 \omega_{p}-\omega_{s}$. Yet, due to the FWM, there will also be an additional wavelength $\omega_{i 2}$ generated at $2 \omega_{s}-\omega_{p}$. This may be undesirable as it will introduce cross talk if there is a channel existing at $\omega_{i 2}$. As for DFG in $\chi^{2}$, there is no cross talk introduced in the similar fashion as FWM - there will only be one converted idler generated at the wavelength, $\omega_{p}-\omega_{s}$. Therefore, parametric process with DFG in $\chi^{2}$ is an effective approach and technique towards the wavelength conversion requirement of the WDM with QAM in the all-optical network. 


\subsection{Existing limitations of current $\chi^{2}$ and $\chi^{3}$ Medium as optical parametric devices}

Currently, optical parametric devices have been demonstrated with both $\chi^{2}$ and $\chi^{3}$ medium. There are many different material systems, such as bulk nonlinear crystal, fiber, waveguide, that have been experimented and demonstrated the capability as an optical parametric device. There is a review of the various optical parametric systems:

\subsubsection{Types of Optical Parametric systems}

\section{Bulk Crystal based}

Crystals without inversion symmetry will have $\chi^{2}$ susceptibility. Lithium niobate $\left(\mathrm{LiNbO}_{3}\right)$, potassium titanate phosphate (KTP), and barium borate (BBO), are some examples. They are excellent candidates to perform nonlinear processes. Their demonstration of parametric processes are the cornerstone of proving the possibility in wavelength conversion as well as optical amplification. The first demonstration of the optical parametric oscillator is dated back to 1965 by Giordmaine and Millers, where they have demonstrate a Optical Parametric Oscillator (OPO) with $\mathrm{LiNbO}_{3}$ [48]. Since then, bulk crystal has continuously pushing various boundaries. One of the major works is the first experimental demonstration of noiseless amplification[49], which has significant implication for communication link in optical network. As for mid-IR generation, a widely tunable source from $2 \mu \mathrm{m}$ to $9 \mu \mathrm{m}$ has been experimentally demonstrated with a bulk QuasiPhasematching (QPM) GaAs OPO[50]. However, such bulk crystal implementations are usually limited in laboratory setting. Bulk-based systems usually consist of a table top implementation with delicate alignment. It is also very expensive due to the amount of optical components and stability required. Due to their lack of integration capability and scalability, bulk crystal implementations are not suitable for optical parametric devices 
for fiber network integration or portable monolithic integration.

\section{Fiber based}

Optical Fiber, which is made of silica, is the major component in the optical network. It is essentially the channel that empowers the optical communication. Silica has no $\chi^{2}$ susceptibility due to its centro-symmetrical structure. However, it possesses third order optical nonlinearity $\chi^{3}$. The optical parametric system can then be easily integrated with existing optical fiber network. Optical parametric amplification[51] and wavelength conversion[52] has been demonstrated in fiber system. However, conventional fiber has a weak nonlinearity. Due to its inherently weak conversion efficiency, it was not a promising choice. To compensate for this issue and promote the fiber-based parametric process, highly nonlinear fiber is designed and fabricated. This highly nonlinear fiber, such as photonics crystal based, has a very high nonlinearity for $\chi 3$ due to the smaller confinement of the light within the core[53]. From this enhancement, it has proven to be a very capable fiber-based parametric system. From [54], it has demonstrated a $-16 \mathrm{~dB}$ conversion efficiency and 40nm bandwidth with a Photonic Crystal Fiber (PCF). Also, with the highly nonlinear fiber, it can also parametrically amplified the signal with 10dB gain and 100nm of bandwidth [51]. But the fiber being used was over 100m and likely very expensive. Even though highly nonlinear fiber can support more efficient parametric conversion, it remains to be very expensive. It also requires an external pump solution with phase modulation to power the nonlinear process and suppress the stimulated Brillioun scattering (SBS). In addition, the use of EDFA to amplify the pump will add additional spontaneous noise to the system and degrade the Noise Figure (NF). Additional high contrast optical filter is also required to remove the high power pump. Therefore, this fiber based optical parametric platform fundamentally lacks the scalability due to the length requirement as well as cost. As a result, it is unlikely to achieve a low power, low cost, highly efficient and portable integrated design. 


\section{Waveguide based}

In waveguide types of design, optical parametric devices have been realized in both $\chi^{2}$ and $\chi^{3}$. Compared to the fiber based and bulk based system, waveguide based has the advantage of integration and mass production. It is more suitable candidate for the goal of portability and scalability.

For $\chi^{3}$ systems, silicon nanowaveguide built on Silicon-on-Insulator is commonly used as the nonlinear device[55][56]. Since it is based on silicon photonics, it has the potential to integrate with CMOS technology. With the silicon nanowaveguide, the interaction length can be much smaller compared to fiber due to the nonlinear enhancement from the strongly confined mode profile. For wavelength conversion, it has demonstrated a $-9.7 \mathrm{~dB}$ conversion efficiency, and a wide band of 100nm 3dB bandwidth [56]. The demonstrated system is on Silicon on Insulator (SOI) to improve the confinement within the silicon. SOI is proven to be more costly to fabricate as well. Like all $\chi^{3}$ based system, Self-Phase Modulation (SPM), XPM, Two-photon Absorption (2PA) and potential cross-talk arised from FWM will all degrade the intended interaction, imposing an effective limit on the parametric performance.

In $\chi^{2}$, Periodically Poled Lithium Niobate (PPLN) has been demonstrated to use as the waveguide platform for parametric processes[57]. Lithium niobate is a strong $\chi^{2}$ material, but its lack of birefringence requires the structure to be periodic poled in order for it to achieve phasematching. This QPM structure has proven to be able to be very capable optical parametric device. It has demonstrated a $-7.5 \mathrm{~dB}$ conversion efficiency with PPLN in $\mathrm{C}$ band with QAM format[57]. It has also demonstrated phase-sensitive amplification[58]. However, this waveguide platform has a low capability in integration and compatibility due to the lack of active designs, such as laser and detectors. As a result, a monolithic solution for PPLN platform is not available. This platform will likely need to be pigtailed into a different platform as the input laser and output detector will be on a different platform. This will impose a scalability issue for the PPLN material 
system, and unsuitable for our low power, monolithically integrated optical parametric device.

The use of semiconductor as the platform for parametric process is preferred due to its small sized, its nonlinear enhancement from strong confinement, and its possibility for integration in a completed optical photonics circuit platform. It can, therefore, have much broader applications than its fiber and bulk counterpart. For all nonlinear processes, the amplification of the signal and efficiency of wavelength conversion originates from the pump laser. Ideally, the nonlinear platform should have the capability to integrate the active design monolithically. In comparison, pump source laser required for the previously demonstrated optical parametric system, such as silicon and PPLN-based, will likely be externally injected. For Silicon, it can be integrated with a laser through direct bonding with III-V semiconductor[59], but it is challenging and expensive. As for PPLN waveguide, it alone does not have active design.

For a target of lower power, small, portable, and monolithic design, a semiconductor platform that has a strong $\chi^{2}$ and can be integrated with a pump laser on the same platform is much preferred. GaAs-AlGaAs material platform has a strong $\chi^{2}$ susceptibility. Its active designs with Indium (InGaAs/AlGaAs) have also been well developed and demonstrated[60][61]. By utilizing this material system, a monolithic integrated solution for both the active and passive nonlinear device can be achieved. However, its lack of birefringence makes it impossible to efficiently phasematch. To leverage such material platform and tackle the phasematching issue, a solution has been proposed using the Bragg Reflection Waveguide[62].

\subsection{Motivation}

Bragg Reflection Waveguide design has shown the capability to leverage the high $\chi^{2}$ nonlinearity in the AlGaAs platform. It allows the GaAs/AlGaAs platform to achieve 
phasematching. This opens the door for all $\chi^{2}$ nonlinear processes in a semiconductor integrated platform where active and passive designs are well developed and demonstrated. This would allow the integration of nonlinear process such as DFG for applications stated in previous section, active design such as pump laser and photodetector for electrically pumping and detection, and passive design such a directional coupler and grating for manipulation of the signal. Bragg Reflection waveguide, therefore, has the strong potential to function as the semiconductor platform where a monolithically integrated design can be achieved. In particular, it leads to the potential for optical parametric devices with monolithic integrated pump source. It can also fill in the gap for optical network as an excellent optical parametric device, where it can function as an optical amplifier in a photonics integrated circuit with low noise figure as well as a wavelength converter for the optical communication technology.

However, there are a number of obstacles remains to be tackled regarding the realization of a highly efficient optical parametric device. Firstly, BRW has a limited acceptance bandwidth of phasematching. This limits the application potential as only works for a narrow pump wavelength. To alleviate the constraints imposed by the structure, tapering as an acceptance bandwidth enhancement has been theoretically proposed for BRW but not thoroughly characterized. Secondly, BRW as an optical parametric amplifier has not been well explored previously. The extreme range of BRW tuning curve design has not been experimentally demonstrated as well. Lastly, a new generation of BRL wafer which has been designed to achieve self-pumping process has been grown. However, the phasematching position and lasing wavelength can be modified by the current and the temperature with different degree. In order to achieve the goal of self-pumping operation from the BRL design, the laser characteristics as well as the nonlinear performance must be characterized to understand the dynamics of the operation regime at different temperature and current level. This will allow the device to achieve self-pumping and demonstrate its potential. This thesis is aimed to resolve the above stated obstacle and 
demonstrate the potential BRW platform as a highly efficient optical parametric device.

\subsection{Thesis Overview}

The aim of this thesis is to utilize Bragg Reflection Waveguide as a platform for the development of Optical parametric devices, for the purpose of optical parametric amplification and optical frequency conversion. The thesis is organized as follows:

In Chapter 2, the background knowledge regarding Bragg Reflection waveguide, optical parametric amplification is reviewed. It includes the discussion of the theory of Bragg Reflection Waveguide, theory of optical parametric process and nonlinear Schrodinger equation that describes the optical parametric process behavior. Chapter 3 investigates the phasematching bandwidth enhancement of the structure and its potential advantages in amplifier and in frequency conversion. Chapter 4 explores the parametric gain of the Bragg Reflection Waveguide devices in both continuous wave and pulsed pump setting. Chapter 5 explores the parametric process in the Bragg Reflection Laser devices which provides the parametric gain in a self-pumped setting. Finally, Chapter 6 concludes and summaries this thesis and future direction of this research is discussed. 


\section{Chapter 2}

\section{Background}

Nonlinear processes have profound applications in many aspects. To facilitate the applicability in sensing and in telecommunication, a platform which has a strong optical nonlinearity as well as ability for monolithic integration with laser and detector is strongly desired. With a monolithic platform solution, many application solutions can be designed to be efficient, low-power, scalable, and even portable. Bragg Reflection waveguide[62] is a platform that can satisfy the aforementioned requirements as it is based on AlGaAs material, where many monolithic solutions such as lasers and detectors have been well developed. In this section, background theory of nonlinear parametric process as well as knowledge regarding Bragg Reflection Waveguide will be introduced.

\subsection{Nonlinear Optical Parametric processes and its applications}

Nonlinear Optical parametric process is the nonlinear interaction of light that does not rely on transitions between energy states. It allows phenomena such as difference fre-

quency generation and sum frequency generation without changing the energy state of the system. Materials with $\chi^{2}$ are commonly utilized as the medium for nonlinear optical 
processes.

Difference Frequency Generation is the process that allows the applications such as optical parametric amplification and wavelength conversion. In this process as shown in Figure 2.1a, two light sources are injected into the nonlinear medium and a third light is generated. The frequency of the generated light will have the difference of frequency between the two injected light source. These three interacting waves are commonly denoted as pump, signal and idler, where pump and signal are injected sources, and the idler is the nonlinearly generated light. To facilitate the nonlinear processes such as DFG to interact efficiently, the three waves that are interacting in the process must satisfy the energy conservation and the phasematching condition.

\section{Energy Conservation}

For energy conservation, the interacting waves must satisfy the following equation

$$
E_{p}=E_{s}+E_{i}
$$

Where $E_{p}$ is the energy of the pump, $E_{s}$ is the energy of the signal and $E_{i}$ is the energy of the idler. Since there is a well defined relationship between energy and frequency, which is given by :

$$
E=\hbar \omega
$$

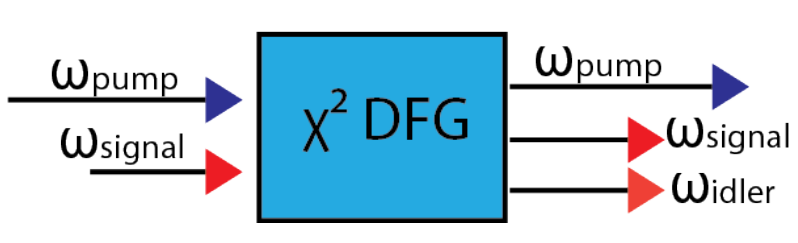

(a)

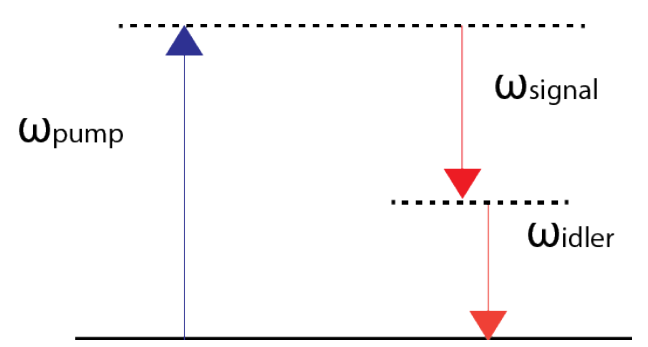

(b)

Figure 2.1: Symbolic Diagram for DFG. Two sources, the pump and the signal, are injected into the $\chi^{2}$ medium to perform DFG. An addition wavelength, the idler, is generated. 
where $\hbar$ is the planck constant and $\omega$ is the angular frequency of the wave. The energy conservation condition is commonly written as the following:

$$
\hbar \omega_{p}=\hbar \omega_{s}+\hbar \omega_{i}
$$

which can be rewritten into:

$$
\omega_{p}=\omega_{s}+\omega_{i}
$$

The energy diagram for DFG is shown in Figure 2.1b. This equation suggests an interesting phenomena, as by tuning the signal wavelength, the idler wavelength can be correspondingly adjusted and generated efficiently as long as the phasematching condition is satisfied. If the signal wavelength is close to double the wavelength of the pump, the idler will also be similar to that wavelength as well. The position where wavelengths of the signal and idler coincides:

$$
\omega_{s}=\omega_{i}
$$

This condition is called the degeneracy. However, if the signal injected is detuned very far from degeneracy point, the idler generated can be detuned to a very far extent as well. This effect can be leveraged to generate widely tunable mid-IR radiation, where the signal which is far from the degeneracy point is slightly tuned to generate a much longer wavelength for the idler. This effect can be seen on the tuning curve shown in Figure 2.2, where a 50nm detune of the signal and result in 90nm of the idler. SH3 Tuning Curve Leverage

\section{The Phasematching Condition}

For the phasematching condition, the interacting waves must satisfy the following equation:

$$
k_{p}=k_{s}+k_{i}
$$




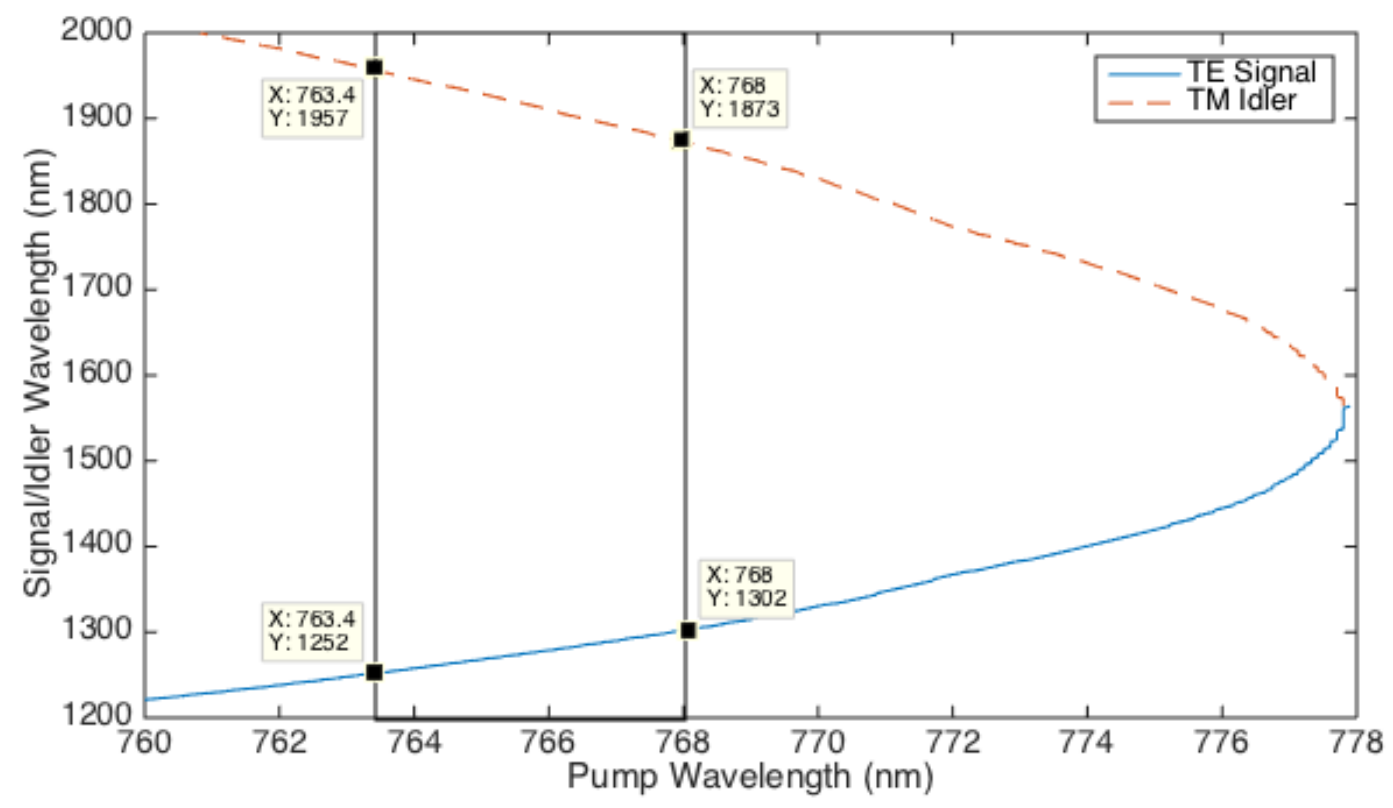

Figure 2.2: Tuning Curve of the BRW Structure.

Where $k_{p}$ is the propagation constant of the pump, $k_{s}$ is the propagation constant of the signal and $k_{i}$ is the propagation constant of the idler. The propagation constant has the following definition:

$$
k=2 \pi n(\lambda) / \lambda
$$

The propagation constant has a direct relationship between the wavelength and the refractive index. Due to chromatic dispersion, not all wavelength can be phasematched. As a result, depending on the material properties, the refractive indices of the interacting wavelength will be different and there will be only a range of wavelength interaction that is feasible. This range is described by the tuning curve and the phasematching bandwidth. The tuning curve indicates the optimum phasematching position given a specific pump wavelength while the phasematching bandwidth indicates the bandwidth at which the nonlinear conversion remains efficient.

For the nonlinear interaction to be efficient, the waves are required to maintain a constant phase relationship. However, if it is not phasematch, it will limit the effective 
length of interaction due to the destructive interference of the nonlinear generation. This condition put a constraint on the phasematching bandwidth - bandwidth within which the nonlinear conversion remains efficient. Given a material, not all wavelength are likely to be satisfied the phasematching condition due to chromatic dispersion. As a result, for this phasematching condition to be satisfied at the desired operation point, special techniques are utilized.

\subsubsection{Phasematching Techniques}

In bulk crystal, birefringence properties is commonly used to achieve phasematching. This method is called Birefringent Phasematching. Due to the significant difference in the index between the ordinary orientation and the extra ordinary orientation of the crystal, one can achieve phasematching by inject light with different polarizations. This allows different wavelength to achieve phasematching

Another approach is Quasi-Phasematching Techniques. In $\chi^{2}$ process such as difference frequency generation, idler can still be generated even with a phasemismatch. If the phasemismatch is not corrected, the nonlinear generation will start to destructively interfere and limit the performance of the nonlinear conversion. By periodically switching the $\chi^{2}$ coefficient, one can allow efficient nonlinear conversion with a phase mismatch. This phase mismatch is corrected by a $\chi^{2}$ Grating. In Domain-reversal QPM, the phase mismatch is periodically corrected by having $\chi^{2}$ switching signs along the propagation. One of the resultant application of this technique is the PPLN. Lithium niobates cannot natively perform nonlinear conversion at around 780nm. However, by perioidically poling the structure, QPM can be achieved. PPLN can perform nonlinear conversion at degeneracy with 780nm pump wavelength. This allows the efficient nonlinear conversion even when the phase mismatch is very high.

Modal phasematching is a technique where a higher order mode is utilized for phasematching. Since a higher order mode has a much lower effective index, one can achieve 
exact phasematching through this method. But in general, higher order mode is difficult to excite and has a higher loss in comparison to the fundamental mode. Special design is needed to take into consideration to achieve this type of high order mode excitation.

\subsubsection{Difference frequency Generation Mathematical represen- tation}

The process of DFG can be viewed as a photon of the pump is split into the one single photon of signal and a single photon of idler. But for a rigorous description, a mathematical expression is needed. To mathematically represent the behavior of DFG process, coupled mode equations derived from the Maxwell equations are commonly used to depict the process. First, lets assume slowly varying amplitude approximation, the following formulation of the propagating waves

$$
\mathbf{E}(x, y, z, t)=A(z) E(x, y) e^{i(k z-w t)}
$$

where $A(z)$ is the slow varying envelope function of the propagation direction $z, E(x, y)$ is the transverse field profile

The coupled mode equations are given as following in [6]:

$$
\begin{aligned}
\frac{d A_{p}}{d z} & =\frac{2 i \omega_{p}^{2} d_{e f f}}{k_{p} c^{2}} A_{s} A_{i} e^{i \Delta k z} \\
\frac{d A_{s}}{d z} & =\frac{2 i \omega_{s}^{2} d_{e f f}}{k_{s} c^{2}} A_{p} A_{i}^{*} e^{i \Delta k z} \\
\frac{d A_{i}}{d z} & =\frac{2 i \omega_{i}^{2} d_{e f f}}{k_{i} c^{2}} A_{p} A_{s}^{*} e^{i \Delta k z}
\end{aligned}
$$

From the coupled-mode equation, one can do some basic assumptions to better understand the DFG process. The pump power can be assumed to be non-depleted. This means that the pump power remains relatively constant over the complete course of interaction. Also we can safely assume the interaction to be phasematched. The assumptions 
can be formulated as following:

$$
\begin{gathered}
\frac{d A_{p}}{d z}=0 \\
\Delta k=0
\end{gathered}
$$

With these assumptions in mind, the equations can then be rewritten into the following form:

$$
\begin{aligned}
& \frac{d A_{s}}{d z}=\left[\frac{2 i \omega_{s}^{2} d_{e f f}}{k_{s} c^{2}} A_{p}\right] A_{i}^{*} \\
& \frac{d A_{i}}{d z}=\left[\frac{2 i \omega_{i}^{2} d_{e f f}}{k_{i} c^{2}} A_{p}\right] A_{s}^{*}
\end{aligned}
$$

These equations can be understood with a simplistic way of looking at it. The terms within the square bracket is constant in the equation above. It suggests that the rate at which the signal and idler are generated is directly related to the effective nonlinear coefficient, and the injected pump power. As a result, to efficiently facilitate the DFG process in a phasematched condition, the medium is required to have a strong nonlinearity to promote a large nonlinear conversion term; the pump power must be strong to accelerate the conversion. By differentiating Equation 2.10a and substituting the conjucate of Equation 2.10b, the following solution for the signal can be obtained.

$$
\begin{aligned}
\frac{d^{2} A_{s}}{d z^{2}} & =\kappa^{2} A_{s} \\
\frac{d^{2} A_{i}}{d z^{2}} & =\kappa^{2} A_{i}
\end{aligned}
$$

where

$$
\kappa=\frac{2 i \omega_{s} \omega_{i} d_{e f f}}{\sqrt{k_{s} k_{i}} c^{2}}\left|A_{p}\right|
$$

By solving the differential equation (2.11a) and (2.11b), and assuming $A_{i}=0$, the 
following equation can be solved:

$$
\begin{gathered}
A_{s}(z)=A_{s}(0) \cosh (\kappa z) \\
A_{i}(z)=i\left(\frac{\omega_{s} n_{i}}{n_{s} \omega_{i}}\right)^{1 / 2} \frac{A_{p}}{\left|A_{p}\right|} A_{1}^{*}(0) \sinh (\kappa z)
\end{gathered}
$$

The result based on Equation 2.13 are plotted in Figure 2.3. There is an exponential growth of the power of the signal and idler as one propagate in the non-depleting pump and phasematching regime. Therefore, signal and idler will be amplified as long as an efficient DFG has been facilitated.

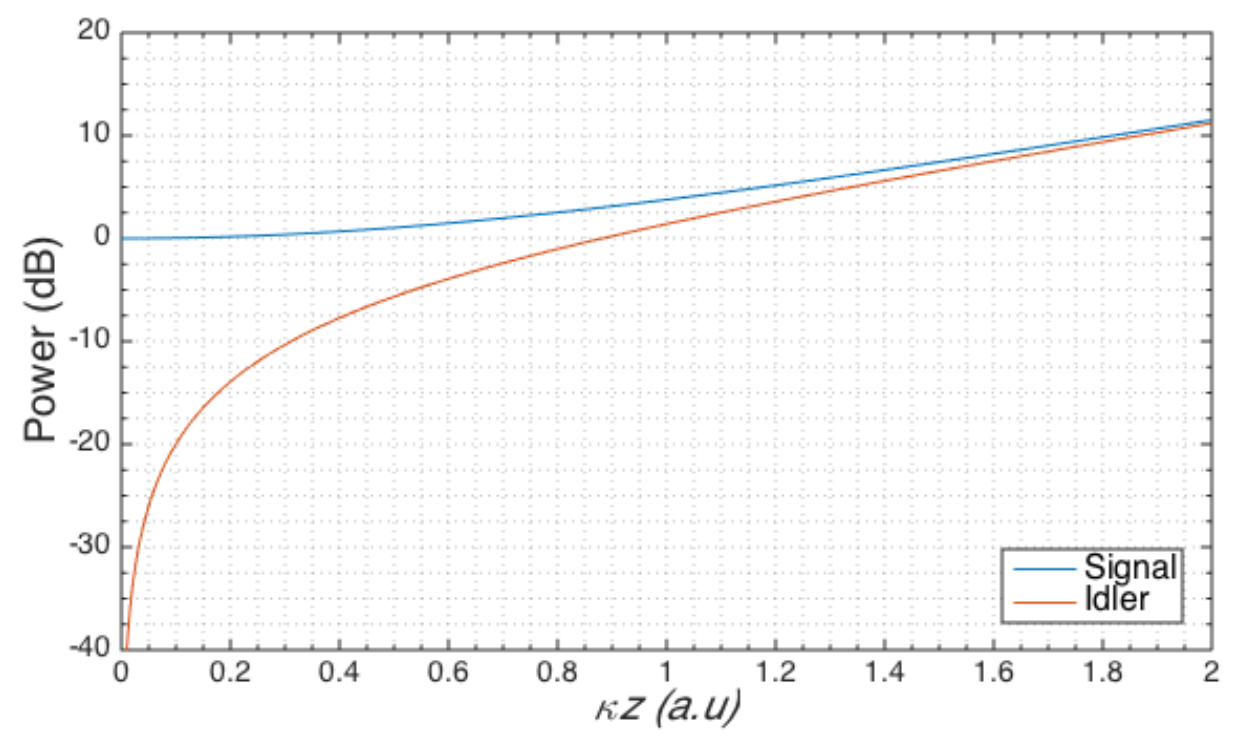

Figure 2.3: Simulated Results based on the solution from Equation 2.13

\subsubsection{Difference Frequency Generation Applications}

Optical parametric amplification is achieved with the DFG process. From the derivation above, it signifies that the signal will be amplified through this process. Moreover, an idler which has the difference in energy between the pump and signal is also generated along with the process. This can in turn be utilized to generate wavelength into the difficult to access mid-IR regime or wavelength conversion in telecommunication. For 
this multi-purpose process to be widely adopted in telecommunication and spectroscopy, a monolithic integrated platform is desired. Bragg Reflection Waveguide satisfies such requirements for the purpose of optical parametric device and we will discuss the background for this platform.

\subsection{Bragg Reflection Waveguide as a monolithic non- linear platform}

Bragg Reflection Waveguide (BRW) is a promising platform for monolithically integrated optoelectronics integrated circuit, especially for $\chi^{2}$ Application. GaAs has a strong $\chi^{2}$ susceptibility; however, it is an isotropic material. Due to strong dispersion behavior of the material, Birefringent Phasematching (BPM) is not natively possible to achieve. As a result, phasematching techniques is needed. QPM in AlGaAs has been demonstrated but it removes the possibility of a monolithic solution. With Bragg reflection waveguide design, the phasematching does not utilize BPM or QPM. It allows efficient nonlinear conversion through the use of exact modal phasematching. The exact modal phasematching is possible due to the guiding of a higher order mode which is shown in Figure 2.4, in conjunction with the conventional TIR mode. This particular mode is referred as Bragg mode. Bragg mode has a much lower effective refractive index than its fundamental mode counterpart, yet the mode profile is able to be tailored by the structure itself. By properly designing the BRW structure, the effective refractive index as well as the mode profile can be tuned to allow the exact modal phasematching and facilitate an efficient nonlinear conversion.

\subsubsection{Bragg Reflection Waveguide Structural details}

BRW is composed of two Quarter-Wave Stacks and a guiding core layer in between. It is grown by Metall Organic Chemical Vapor Deposition (MOCVD) to deposit layers 

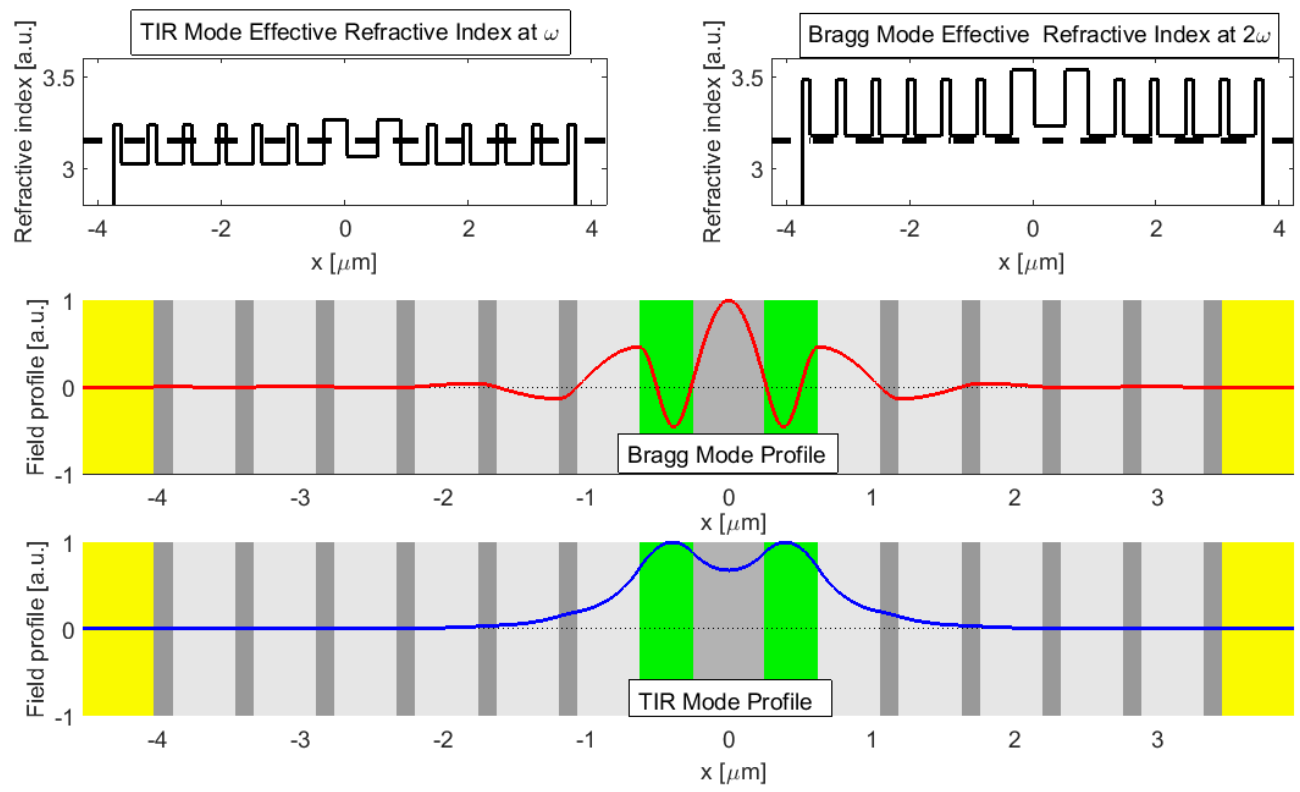

Figure 2.4: Bragg Reflection Waveguide Modal Structure. Top Left: Effective index of the fundamental mode. Top Right: Effective index of the Bragg mode. Middle: Bragg Mode field Profile at $2 \omega$. Bottom: TIR Mode field profile at $\omega$

of $A l_{x} G a_{1-x} A s$ with different concentrations $x$ to create a $1 \mathrm{D}$ Photonic crystal. This 1D structure has a periodic structure of refractive indices that will strongly modify the dispersion behavior. The Quarter-Wave Stacks functioning as a Bragg mirror will reflect the light back. Structurally, it is very similar to VCSEL. However, instead of propagation into the plane of the mirror, BRW guides light along the transverse direction of the structure. It is guiding light along the core layer, while reflecting the light from the Bragg mirror structure back to the core layer. The effective refractive index of this Bragg mode is lower than the lowest refractive index and is at the same value as the Total Internal Reflection (TIR) Fundamental mode. Therefore, BRW design allows the possibility to design the Bragg mode that satisfies the phasetmatching condition.

\subsubsection{Bragg Reflection Waveguide as a nonlinear medium}

A type-II DFG process within the BRW structure is shown in Figure 2.5. The pump $775 \mathrm{~nm}$ is in the Bragg mode, while the signal and the idler are in TE TIR mode and TM 
TIR mode respectively. This process satisfies the energy conservation and this structural design also satisfies the following phasematching equation for the interacting waveguide modes.
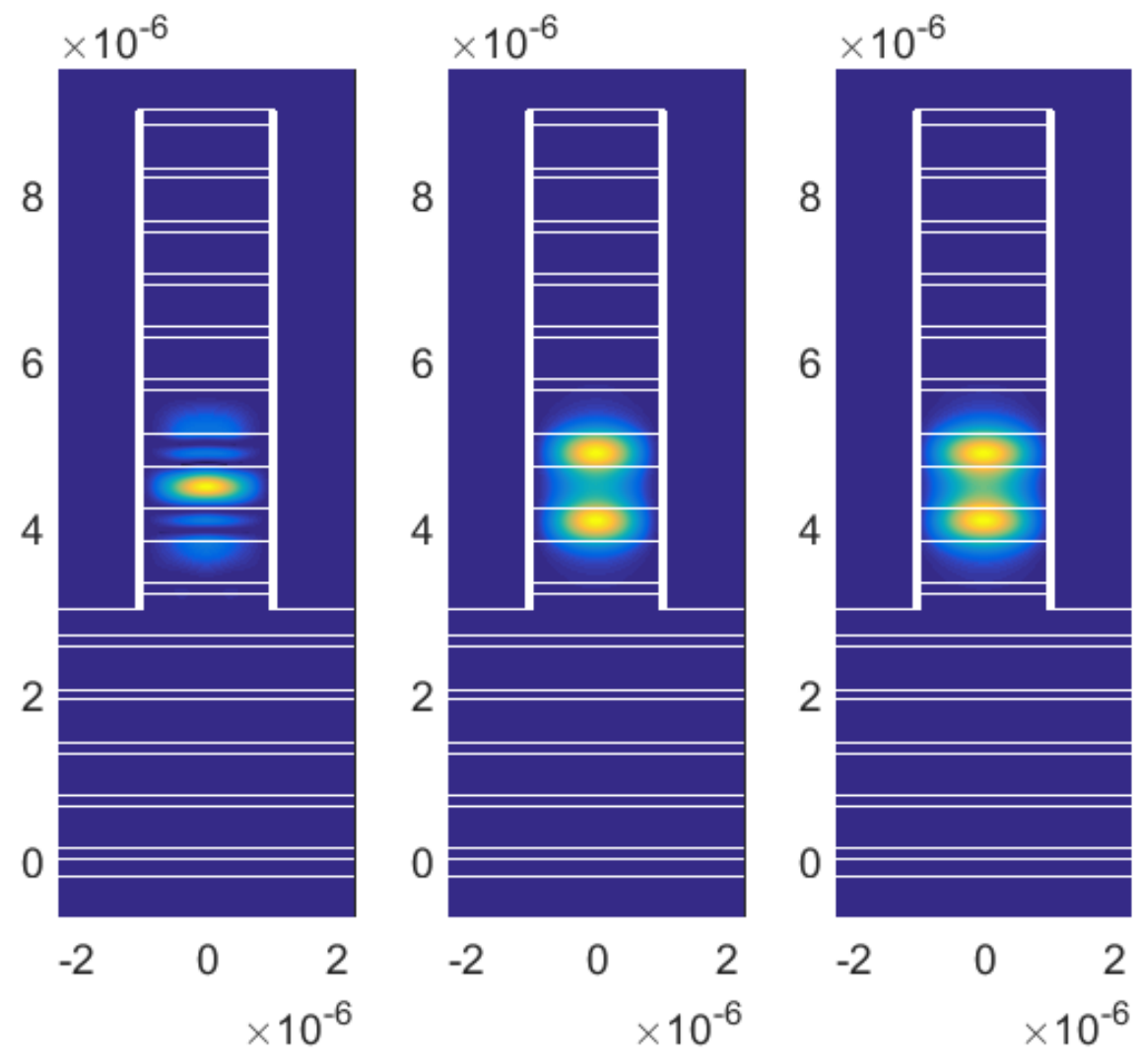

Figure 2.5: 2D Mode Profile of the Type 2 DFG. Left is the Bragg mode with wavelength at $775 \mathrm{~nm}$ guided by the structure. Center is the TE TIR Mode with wavelength 1550nm. Right is the TM TIR Mode with wavelength at 1550nm.

$$
\Delta \beta=\beta_{p}-\beta_{s}-\beta_{i}=0
$$

To determine whether the nonlinear conversion is efficient in this structure, the following coupled-mode equations from PhD Thesis by Payam Abolghasem[63] describes the nonlinear process in waveguide. The propagating waves is defined as:

$$
\mathbf{E}_{\sigma}(x, y, z, t)=A_{\sigma}(z) E_{\sigma}(x, y) e^{i\left(w t-\beta_{\sigma} z\right)}, \sigma \in\{p, s, i\}
$$


where $\mathrm{A}$ is the slow varying envelope function along the waveguide, $\mathrm{E}$ is the transverse mode profile within the Bragg Structure of the wave, $\beta$ is the propagation constant of the corresponding wave. The Coupled-mode equation can be written as the following:

$$
\begin{aligned}
& \frac{\partial A_{p}}{\partial z}=i \kappa_{p} A_{s} A_{i} e^{i \Delta \beta z} \\
& \frac{\partial A_{s}}{\partial z}=i \kappa_{s} A_{p} A_{i}^{*} e^{i \Delta \beta z} \\
& \frac{\partial A_{i}}{\partial z}=i \kappa_{i} A_{p} A_{i}^{*} e^{i \Delta \beta z}
\end{aligned}
$$

$\kappa_{p, s, i}$ from the above equations are defined as:

$$
\kappa_{\sigma}=\left(\frac{8 \pi^{2} d_{e f f}^{2} \xi^{2}}{n_{s} n_{i} n_{p} \epsilon_{0} c \lambda_{\sigma}^{2}}\right)^{1 / 2}, \sigma \in\{p, s, i\}
$$

where $\xi$ is defined as:

$$
\xi=\frac{\iint_{-\infty}^{+\infty} E_{p}(x, y) E_{s}(x, y) E_{i}(x, y) d x d y}{\left.\left.\left(\iint_{-\infty}^{+\infty}\left|E_{p}(x, y)\right|^{2} d x d y\right)^{1 / 2} \iint_{-\infty}^{+\infty}\left|E_{s}(x, y)\right|^{2} d x d y\right)^{1 / 2} \iint_{-\infty}^{+\infty}\left|E_{i}(x, y)\right|^{2} d x d y\right)^{1 / 2}}
$$

and $d_{e f f}$ is defined as:

$$
d_{e f f}=\frac{\iint_{-\infty}^{+\infty} E_{p}^{*}(x, y) d(x, y) E_{s}(x, y) E_{i}(x, y) d x d y}{\iint_{-\infty}^{+\infty} E_{p}^{*}(x, y) E_{s}(x, y) E_{i}(x, y) d x d y}
$$

With the non-depleting pump approximation, the equation 2.16a can be rewritten as:

$$
\frac{\partial P_{p}}{\partial z}=0
$$

With the slowly varying envelope approximation, the following boundary condition for the difference frequency generation process can be defined as:

$$
P_{p}=\left|A_{p}(0)\right|^{2}, P_{s}(0)=\left|A_{s}(0)\right|^{2}, P_{i}(0)=0
$$


The equations describing the power of the interacting waves along the propagation can be derived:

$$
\begin{gathered}
P_{p}(z)=P_{p} \\
P_{s}(z)=P_{s}(0)\left[\cosh ^{2}(g z)+\left(\frac{\Delta \beta}{2 g}\right)^{2} \sinh ^{2}(g z)\right] \\
P_{i}(z)=\frac{\kappa_{i} \xi^{2} P_{p} P_{s}(0)}{g^{2}} \sinh ^{2}(g z)
\end{gathered}
$$

where $g$ parameter is defined as:

$$
g=\sqrt{\kappa_{s} \kappa_{i}|\xi|^{2} P_{p}-\left(\frac{\Delta \beta}{2}\right)^{2}}
$$

To facilitate an efficient nonlinear conversion, it can be inferred from the equation above that the parameter $g$ should be large. This would require that the $d_{\text {eff }}$ and overlapping factor $\xi$ to be high in value. Also, the phase mismatch should be zero. Therefore, BRW design must take into account of the corresponding $d_{\text {eff }}$ and overlaping factor $\xi$ as well as the effective indices for all three waves. Therefore, the goal of BRW design is to facilitate the guiding of the Bragg mode which can satisfy the phasematching and a maximum overlapping with the other interacting waves.

In Figure2.6, an optimized design of Bragg Reflection Waveguide is shown. In this structure, the majority of the power of Bragg Mode is guiding at the center of the core, while the TIR mode for the signal and idler is guiding within the higher refractive index regime just beside the core. This promotes the confinement and overlapping of TIR and Bragg mode within the multi-layer core area. The higher confinement would increase the intensity within the waveguide and the higher overlapping would improve the overlapping factor $\xi$. Such design would lead to higher conversion efficiency. Various other parameters such as layer thickness, aluminum concentration, number of Quarter-Wave Layers and operating wavelength can be modified. Correspondingly, the modified design 

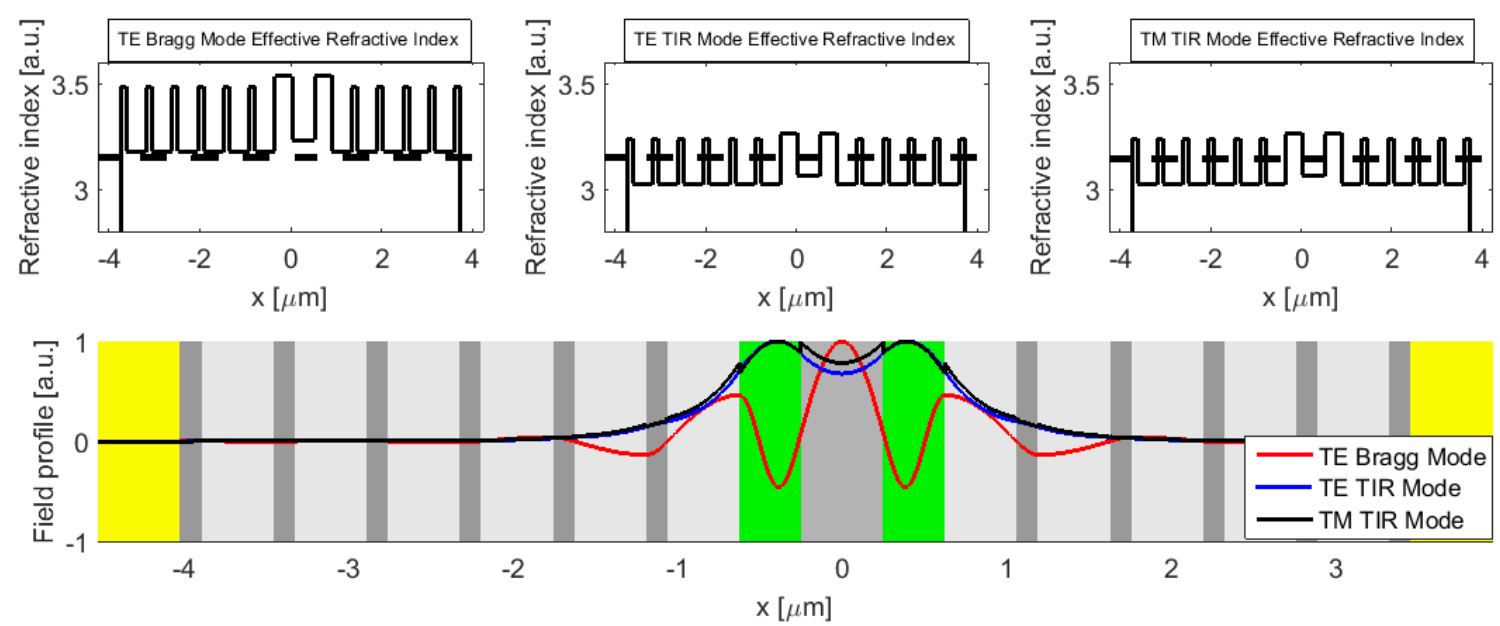

Figure 2.6: 1D BRW Modal Analysis. Top Left: Effective index of the TE Bragg Mode at $2 \omega$. Top Middle: Effective Index of TE TIR Mode at $\omega$. Top Right: Effective index of the TM TIR Mode at $\omega$. Bottom: Modal profiles of each modes.

can, therefore, be changed as needed to adapt and adjust to the required performance.

Nonlinear Performances demonstrations of such design have previously reported in [62]. By injecting 1550nm continuous wave source into the Bragg Reflection Waveguide, Type-II SHG has been reported and have a normalized conversion efficiency of 6.30 $\% \mathrm{~W}^{-} 1 \mathrm{~cm}^{-2}$. Difference Frequency Generation has also been reported in [62] by coupling the pump from Ti:Sapphire CW Laser and the signal with a C-Band tunable Source. By demonstrating the capability of the performing DFG in the BRW, it also indicates that it is a potential platform for the optical parametric device.

\subsection{Bragg Reflection Waveguide Diode Laser}

In order to perform nonlinear conversion such as difference frequency generation, the energy as the pump would require to be propagating in the Bragg mode. The higher the pump power guided in Bragg mode within the waveguide, the stronger the conversion will be as denoted by the parameter $g$ in Equation 2.22. However, there is a limit in amount of power in coupling the pump power into the waveguide. There are primarily two reasons for the limitation. 
The primary reason is the low efficiency in coupling. For the pump wavelength, which is at $775 \mathrm{~nm}$, the fundamental TIR mode for the $775 \mathrm{~nm}$ also exists within the structure. Any energy in the TIR mode at the pump wavelength would be rendered uneffective towards nonlinear conversion because it cannot be phasematched due to dispersion. Since the Bragg mode is similar to a higher order mode, it has a low overlapping with a fundamental Gaussian mode. As a result, using a conventional laser with Gaussian mode output and free space butt-coupling into the waveguide would result in a low efficacy as the majority of the power will probably not coupled into the Bragg mode.

The secondary reason is that the pump wavelength, $775 \mathrm{~nm}$, is also absorptive in AlGaAs due to the energy proximity towards the native bandgap of the structure. A large amount of energy can be absorbed and transferred to thermal energy. This poses a limit on the input power as the waveguide facet can be damaged from the high intensity of the focused pump.

The more power coupled into the Bragg mode instead of the TIR mode, the better the nonlinear conversion becomes. To relieve such constraints within the BRW platform, it is motivated to create a laser which generates the light with Bragg Mode at the pump wavelength and maintain a high intensity within the waveguide. This type of laser is referred as Bragg Reflection Waveguide Diode Laser.

Bragg Reflection Waveguide Laser Diode (BRL) has been demonstrated in [64]. By lasing in Bragg mode, it can allow the high intensity of the pump in the Bragg mode within the structure as well as the suppression of the TIR mode. This will be able to resolve the two major issues that was discussed for the BRW structure, as it can have a high power without coupling difficulties that we have previously discussed. By incorporating the laser with the BRW platform, the nonlinear conversion can be facilitated by the pump generated with electrical current injection. This self-pumped BRW design form an important step for the monolithic integration requirement for many applications.

To create a diode laser, a p-n junction, a gain medium, a cavity is necessary. In 
BRL, the p-n junction can be achieved by doping the structure to form a diode junction. Electrical contacts can be deposited on the structure for injection of electrical carriers. By adding a stressed InAlGaAs quantum well in the core layer, it can achieve a population inversion with electrical carrier injection. As a result, the quantum well will function as a gain medium. Cavity can be formed by creating an interface with reflectivity. A simple semiconductor-air interface will have sufficient reflectivity which has about $33 \%$. With all the requirement of the diode laser satisfied, the structure would be able to lase with carrier injection passed the lasing threshold. For a laser, the lasing threshold of a particular mode is described by the following equation:

$$
g(I) \Gamma_{m}-\alpha_{m}=0
$$

where $\alpha_{m}$ is the propagating loss of the mode $m, \mathrm{~g}(\mathrm{I})$ is the gain as a function of current and $\Gamma_{m}$ is the overlapping between the gain region and the mode $m$.

However, there are difficulties in the realization of the Bragg Laser, due to the general lower loss of the TIR mode. Consequently, according to the equation 2.24, the TIR mode is more likely to achieve lasing instead of the Bragg mode because it can easily satisfy this condition. As a result, a special design is needed to increase the gain overlap with Bragg mode while suppressing the gain overlap with the TIR mode.

$$
\text { Ratio }=\frac{\Gamma_{\text {Bragg }}}{\Gamma_{\text {TIR }}}
$$

Once the ratio is designed to be larger than 1, Bragg Reflection structure which lase in Bragg mode can is more likely achieved.

With the ability to achieve lasing in Bragg mode, the BRL structure can also be designed to maximize the overlap with the signal TIR mode and function as a nonlinear medium. Therefore, such structure can be achieved the capability of electrically selfpumping, high nonlinear conversion efficiency, and monolithically integration, which are 
well suited for the optical parametric device purpose.

\subsection{NLSE Solver}

To analyze the performance of the BRW structure, wave propagation simulation is required in addition to 2D mode analysis. Nonlinear Schrolinger Equation Solver (NLSE) is a commonly used tools to model the nonlinear behavior of the interacting propagating waves. It examines effects of $\chi^{2}$ and $\chi^{3}$ as well as the modal dispersion on the interacting waves. Through this solver, we are able to analyze and predict the nonlinear behavior of the optical parametric device in different scenarios, such as continuous wave pump operation, pulse pump operation, or mixed operation.

\subsubsection{Mathematical formulation}

To utilize the model of the NLSE solver, there are a couple assumptions that will need to be established. By assuming the slow varying envelope approximation as well as the confined mode profile, the electric fields of the waves can be represented by

$$
\mathbf{E}_{\sigma}(x, y, z, t)=A_{\sigma}(z, t) E_{\sigma}(x, y) e^{i\left(\beta_{\sigma} z-w_{\sigma} t\right)}, \sigma \in\{p, s, i\}
$$

where $A_{\sigma}$ is the envelope function that describes the spatial and temporal relationship of the waves (pump, signal, and idler).

The evolution of the envelope function $A_{\sigma}$ along the propagation can be modeled by the following set of Nonlinear Schrodinger Equations:

$$
\begin{aligned}
\frac{\partial A_{p}}{\partial z}= & -\frac{1}{2} \alpha_{p} A_{p}-\beta_{1, p} \frac{\partial A_{p}}{\partial t}+i \frac{1}{2} \beta_{2, p} \frac{\partial^{2} A_{p}}{\partial t^{2}}-\frac{1}{6} \beta_{3, p} \frac{\partial^{3} A_{p}}{\partial t^{3}}-i \kappa_{p} A_{i} A_{s} e^{i \Delta \beta z} \\
& -\frac{1}{A_{e f f, p}^{(3)}}\left[\frac{1}{2} \alpha_{2, p}-i \frac{2 \pi n_{2, p}}{\lambda_{p}}\right]\left(2\left|A_{p}\right|^{2}+2\left|A_{s}\right|^{2}+2\left|A_{i}\right|^{2}\right) A_{p}
\end{aligned}
$$




$$
\begin{aligned}
\frac{\partial A_{s}}{\partial z}= & -\frac{1}{2} \alpha_{s} A_{s}-\beta_{1, s} \frac{\partial A_{s}}{\partial t}+i \frac{1}{2} \beta_{2, s} \frac{\partial^{2} A_{s}}{\partial t^{2}}-\frac{1}{6} \beta_{3, s} \frac{\partial^{3} A_{s}}{\partial t^{3}}-i \kappa_{s} A_{p} A_{i}^{*} e^{i \Delta \beta z} \\
& -\frac{1}{A_{e f f, s}^{(3)}}\left[\frac{1}{2} \alpha_{2, s}-i \frac{2 \pi n_{2, s}}{\lambda_{s}}\right]\left(2\left|A_{p}\right|^{2}+2\left|A_{s}\right|^{2}+2\left|A_{i}\right|^{2}\right) A_{s} \\
\frac{\partial A_{i}}{\partial z}= & -\frac{1}{2} \alpha_{i} A_{i}-\beta_{1, i} \frac{\partial A_{i}}{\partial t}+i \frac{1}{2} \beta_{2, i} \frac{\partial^{2} A_{i}}{\partial t^{2}}-\frac{1}{6} \beta_{3, i} \frac{\partial^{3} A_{i}}{\partial t^{3}}-i \kappa_{i} A_{p} A_{s}^{*} e^{i \Delta \beta z} \\
& -\frac{1}{A_{e f f, i}^{(3)}}\left[\frac{1}{2} \alpha_{2, i}-i \frac{2 \pi n_{2, i}}{\lambda_{i}}\right]\left(2\left|A_{p}\right|^{2}+2\left|A_{s}\right|^{2}+2\left|A_{i}\right|^{2}\right) A_{i}
\end{aligned}
$$

where $\alpha_{\sigma}$ is the linear propagation loss of the pump (signal, idler), $\beta_{1, \sigma}, \beta_{2, \sigma}, \beta_{3, \sigma}$ are the first, second and third derivatives of propagation constant respectively at the corresponding wavelength, $\kappa_{\sigma}$ is the nonlinear conversion coefficient given by the equation 2.17, $\Delta \beta$ is the phase mismatch coefficient given by $2.14, A_{e f f, \sigma}^{(3)}$ is the effective area of the propagating mode, $\alpha_{2, \sigma}$ is the two-photon absorption coefficient of wave $\sigma, n_{2, \sigma}$ is the effective $\chi^{3}$ refractive indices of wave $\sigma, \lambda_{\sigma}$ is the wavelength of wave $\sigma$.

The first term of the equation is describing the linear loss of the power of the mode along the propagation. The second, third and forth terms describes the temporal behavior of a pulse envelope such as Group velocity mismatch, group velocity dispersion, and higher order group velocity dispersion. The fifth term describes the DFG process, which relates with the other two interacting waves as well as the nonlinear coefficient. The final ensemble term is describing all the $\chi^{3}$ effects, which includes the two-photon absorption, self-phase modulation and cross-phase modulation.

It is necessary to analyze the nonlinear efficiency of the optical parametric device in NLSE solver because the structure parameters of optical parametric device can significantly change the nonlinear behaviour of the interacting waves. The analysis is especially important for ultra fast regime, where the interacting waves is in pulse envelope. By using the pulsed operation, the interacting waves will have a higher peak power. This will allow a stronger nonlinear interaction as, from Equation 2.23, the higher the peak pump power, the stronger the nonlinear conversion. For Difference Frequency generation, a 
pulsed-pump input with a high peak power can greatly accelerate the nonlinear process. However, when pulse source is utilized, the first and second order dispersion will be crucial to analyze. This is because the first and second order dispersion will cause group velocity mismatch and group velocity dispersion. With the Group velocity mismatch phenomenon, it will cause a temporal walk off between the pump and the signal. The temporal walk off will reduce the temporal overlap among the interacting waves and the conversion efficiency. With the Group velocity dispersion, it will cause temporal pulse broadening and reduce the peak power of the pulse. As a result, such effects may quickly reduce the peak power of the pulse and the conversion efficiency as well. Also, with a higher peak power, the $\chi^{3}$ effects will also be more prominent and affect propagation of the pump due to phenomena such as two photon absorption and Self phase modulation. Both of which are undesirable and limit the nonlinear conversion performance. Therefore, NLSE solver is necessary to help analyze the ultra fast regime and locate the optimum operation point, such as pulse width, pulse strength, and propagation length, for the parametric process. 


\section{Chapter 3}

\section{Bandwidth of optical parametric devices}

Bandwidth is an important factor as a parametric device. It indicates the limits within which the pump and the signal can facilitate the parametric process and the extent of how far an idler can be generated. In $\chi^{3}$ process, such as fiber-based parametric system, the typical bandwidth is at the order of $60 \mathrm{~nm}$ [65]. It does not have a flat band conversion efficiency. In addition, it cannot convert wavelength that is in too close to the pump filter. This may potentially impact the robustness of a fiber-based optical parametric amplifier. However, in $\chi^{2}$ based, such as BRW, it has been, though not completely, shown the flat band characteristics of the idler generation [62]. It has been theorectically predicted to be at the order of 100nm, but has not been experimentally demonstrated. Especially for $\chi^{2}$, there is an acceptance bandwidth limitation, the bandwidth within which the pump can facilitate a nonlinear interaction. In order to achieve a wider acceptance bandwidth, it is possible to deploy a bandwidth enhancement solution to the BRW, and drastically expand the application potential of the Bragg platform. The waveguide ridge can be tapered as a mean to enhance the bandwidth after the growth of the wafer. Although the theory of tapering as a bandwidth enhancement has been developed, a thorough 
characterization on Tapered BRWs have not been demonstrated. In this chapter, the normal and enhanced bandwidth of the BRW will be characterized to demonstrate the capability of tapering. In additon, the conversion bandwidth will also be experimentally characterized to show the flat-band behavior.

\subsection{Bandwidth Enhancement through tapering}

Bandwidth of the phasematching is depending on the dispersion of the material. Given a specific chromatic dispersion relationship, there is only a limited range of bandwidth that can be achieved. However, this dispersion behaviour can be modified through engineering of the waveguide structure. It can satisfy a more versatile requirement by modifying the structural design of the waveguide.

A 2D cross section of a basic BRW waveguide is shown in the Figure 3.2. It can be described by its ridge width and ridge depth. As each waveguide with different ridge depth and ridge width would slightly modify the dispersion of the device, the modal indices of each mode will also change as a result of the structural difference. The phasematching position would shift as a result from the modal indices modification. By leveraging such effect, one can design a waveguide with tapering ridge width and varying etch depth along the propagation direction. This design of tapering can modify the phasematching position along the waveguide. As a result, the resultant effect of this design would effectively create a wider bandwidth at the cost of reduced conversion efficiency.

\section{Background theory of tapering}

The theory of the bandwidth enhancement from the tapering is discussed below, reproduced from PhD Thesis by Dong Peng Kang. If the modification of the effective index is assumed to be linearly related to the ridge width and the tapering is linearly tapered, 
the following equation can be written to described the effective index as a function of propagation distance.

$$
\begin{aligned}
& n_{q}\left(\omega_{q}, z\right) \approx n_{q}\left(\omega_{q}, 0\right)+\gamma_{q}\left(\omega_{q}\right) z \\
& q \in S H T E, F M T E, F M T M
\end{aligned}
$$

where $\mathrm{n}_{q}\left(\omega_{q}\right)$ is the effective refractive indices of the mode $q$ at the $z=0, \gamma_{q}\left(\omega_{q}\right)$ is the linear parameter that relates the modification of the effective index with respect to mode $q$ along the propagation length.

Therefore, for the Type-2 SHG that we are interested in, the phaematching equation can be rewritten as the following with Eq. 3.1.

$$
\begin{aligned}
\Delta k(\omega, z) & =\frac{2 \omega}{c} n_{\text {SHTE }}(2 \omega, z)-\frac{\omega}{c} n_{F M T E}(\omega, z)-\frac{\omega}{c} n_{F M T M}(\omega, z) \\
& =\left[\frac{2 \omega}{c} n_{S H T E}(2 \omega, 0)-\frac{\omega}{c} n_{F M T E}(\omega, 0)-\frac{\omega}{c} n_{F M T M}(\omega, 0)\right] \\
& +\left[\frac{2 \omega}{c} \gamma_{S H T E}(2 \omega) z-\frac{\omega}{c} \gamma_{F M T E}(\omega) z-\frac{\omega}{c} \gamma_{F M T M}(\omega) z\right] \\
& =\Delta k(\omega, 0)+\zeta(\omega) z
\end{aligned}
$$

where

$$
\begin{gathered}
\Delta k(\omega, 0)=\frac{2 \omega}{c} n_{S H T E}(2 \omega, 0)-\frac{\omega}{c} n_{F M T E}(\omega, 0)-\frac{\omega}{c} n_{F M T M}(\omega, 0) \\
\Delta k(\omega, 0)=k_{S H T E}(2 \omega, 0)-k_{F M T E}(\omega, 0)-k_{F M T M}(\omega, 0)
\end{gathered}
$$

and

$$
\zeta(\omega)=\frac{2 \omega}{c} \gamma_{S H T E}(2 \omega) z-\frac{\omega}{c} \gamma_{F M T E}(\omega) z-\frac{\omega}{c} \gamma_{F M T M}(\omega) z
$$

To be able to derive the terms regarding the effective indices of each mode at different wavelength, the effective index $n_{q}(\omega, 0)$ can also be written as the following through 
Taylor expansion:

$$
\begin{aligned}
k_{q}(\omega) & =k_{q}\left(\omega_{0}\right)+\left.\frac{\delta k_{q}}{\delta \omega}\right|_{\omega_{0}}\left(\omega-\omega_{0}\right)+\left.\frac{1}{2} \frac{\delta^{2} k_{q}}{\delta \omega^{2}}\right|_{\omega_{0}}\left(\omega-\omega_{0}\right)^{2} \\
& =k_{q}\left(\omega_{0}\right)+\frac{1}{v_{q \mid \omega_{0}}}\left(\omega-\omega_{0}\right)-\frac{\lambda_{0}^{2}}{4 \pi c} D_{q \mid \omega_{0}}\left(\omega-\omega_{0}\right)^{2}
\end{aligned}
$$

where $k_{q}\left(\omega_{0}\right)$ is the propagation constant at $\omega_{0},\left.v_{q}\right|_{\omega_{0}}$ is the group velocity for mode $q$ at $\omega_{0}$ and is defined as $\left(\left.\frac{\delta k_{q}}{\delta \omega}\right|_{\omega_{0}}\right)^{-1}, \lambda_{0}$ is the wavelength of interested, $\left.D_{q}\right|_{\omega_{0}}$ is the group velocity dispersion parameter at $\omega_{0}$ and is defined as $\left.\frac{2 \pi c}{\lambda_{0}^{2}} \frac{\delta^{2} k_{q}}{\delta \omega^{2}}\right|_{\omega_{0}}$.

Using the Taylor expansion in Eq. 3.4 above and assuming $\omega_{0}$ is the phasematching wavelength at $z=0$ where $\Delta k\left(\omega_{0}, 0\right)=0$. Equation 3.3 can be rewritten as:

$$
\begin{aligned}
\Delta k(\omega, 0) & =\frac{1}{v_{S H T E \mid 2 \omega_{0}}}\left(2 \omega-2 \omega_{0}\right)-\frac{\left(\lambda_{0} / 2\right)^{2}}{4 \pi c} D_{S H T E \mid 2 \omega_{0}}\left(2 \omega-2 \omega_{0}\right)^{2} \\
& -\frac{1}{v_{F M T E \mid \omega_{0}}}\left(\omega-\omega_{0}\right)+\frac{\lambda_{0}^{2}}{4 \pi c} D_{F M T E \mid \omega_{0}}\left(\omega-\omega_{0}\right)^{2} \\
& -\frac{1}{v_{F M T M \mid \omega_{0}}}\left(\omega-\omega_{0}\right)+\frac{\lambda_{0}^{2}}{4 \pi c} D_{F M T M \mid \omega_{0}}\left(\omega-\omega_{0}\right)^{2}
\end{aligned}
$$

If using a small degree of tapering, $\gamma_{S H T E}(2 \omega), \gamma_{F M T E}(\omega)$ and $\gamma_{F M T M}(\omega)$ can be approximate to be a constant, that is:

$$
\begin{aligned}
\gamma_{S H T E}(2 \omega) & \approx \gamma_{S H T E}\left(2 \omega_{0}\right) \\
\gamma_{F M T E}(2 \omega) & \approx \gamma_{F M T E}\left(\omega_{0}\right) \\
\gamma_{F M T M}(2 \omega) & \approx \gamma_{F M T M}\left(\omega_{0}\right) \\
\zeta(\omega) & \approx \zeta(\omega)
\end{aligned}
$$

From Eq. 3.5 and Eq. 3.6, it can be realized that all the parameters such as dispersion and $\gamma$ can be numerical calculated from the numerical solver. With Eq. 3.2, the following phasematching equation can be written and solved: 


$$
\begin{aligned}
\Phi(\omega) & =\int_{0}^{L} d z \exp \left[i \int_{0}^{z} \Delta k\left(\omega, z^{\prime}\right) d z^{\prime}\right] \\
& =\int_{0}^{L} d z \exp \left[i\left(\Delta k(\omega, 0) z+\zeta(\omega) z^{2}\right)\right] \\
& =\frac{\sqrt{\pi}}{2 \sqrt{i \zeta(\omega)}} \exp \left[\frac{i(\Delta k(\omega, 0))^{2}}{2 \zeta(\omega)}\right]\left\{\operatorname{erfi}\left[\frac{\sqrt{i}(\Delta k(\omega, 0)+2 \zeta(\omega) L}{2 \sqrt{\zeta(\omega)}}\right]-\operatorname{erfi}\left[\frac{\sqrt{i \Delta k(\omega, 0)}}{2 \sqrt{\zeta(\omega)}}\right]\right\}
\end{aligned}
$$

where erfi is the imaginary error function, and $\mathrm{L}$ is the propagation length of the tapered waveguide. The SHG power generation curve can then be calculated through the phasematching function as follows:

$$
P(\omega)=|\Phi(\omega)|^{2}
$$

By numerically calculated the solution from Eq. 3.8 using the parameters from numerical solver, the SHG tuning curve of the highly tapered, less tapered and straight waveguide are shown in Figure 3.1. It can be seen that there can be a bandwidth enhancement of 10 times increase from straight to tapered waveguide.

\section{Tapered waveguide Fabrication}

The simple structure of the BRW is shown in 3.2. It is designed to possess a degenerate

phasematching point at around 1560nm. In order to implement the Tapering process of the Bragg reflection waveguides, the following fabrication process demonstrated in Figure 3.3 is utilized and fabricated by Rajiv Prinja. Multiple waveguides with different tapering degree are fabricated on the same chip to achieve the same fabrication quality and device length. Through this way, proper comparisons and contrasts among waveguides with different tapering degrees can be accomplished. In Table 3.1, the structural characteristics of the fabricated tapered waveguide are outlined. 


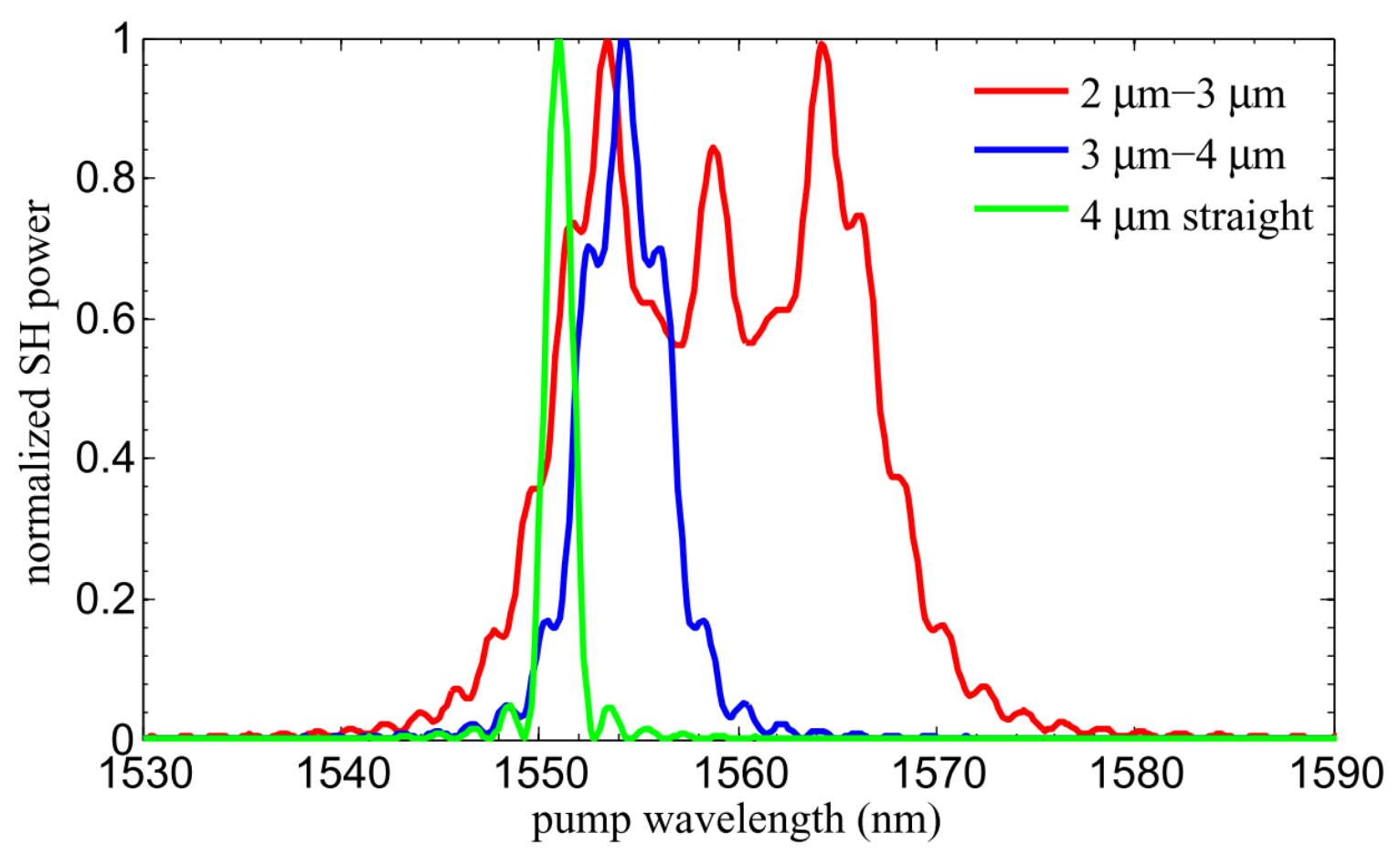

Figure 3.1: Simulated normalized SHG Spectrum, with permission to reproduce from $[66]$

Table 3.1: Structural Information of the Tapered Waveguides

\begin{tabular}{cccc}
\hline \hline Waveguide & Ridge Width $1(\mu m)$ & Ridge Width $2(\mu m)$ & Tapering difference $\left(\frac{R W 2}{R W 1}\right)$ \\
\hline A & 2.7 & 2.7 & 1 \\
B & 2.5 & 2.8 & 1.12 \\
C & 2.4 & 2.9 & 1.20 \\
D & 2.1 & 2.7 & 1.28 \\
E & 1.8 & 2.7 & 1.5 \\
F & 1.5 & 2.5 & 1.67 \\
\hline
\end{tabular}




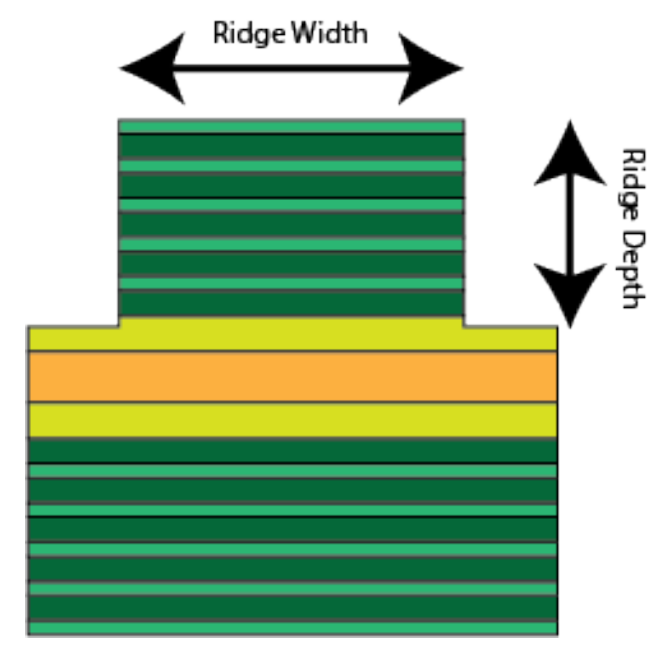

Figure 3.2: 2D Cross section of Bragg Reflection Waveguide Structure. A full description of the structure can be found in [62].

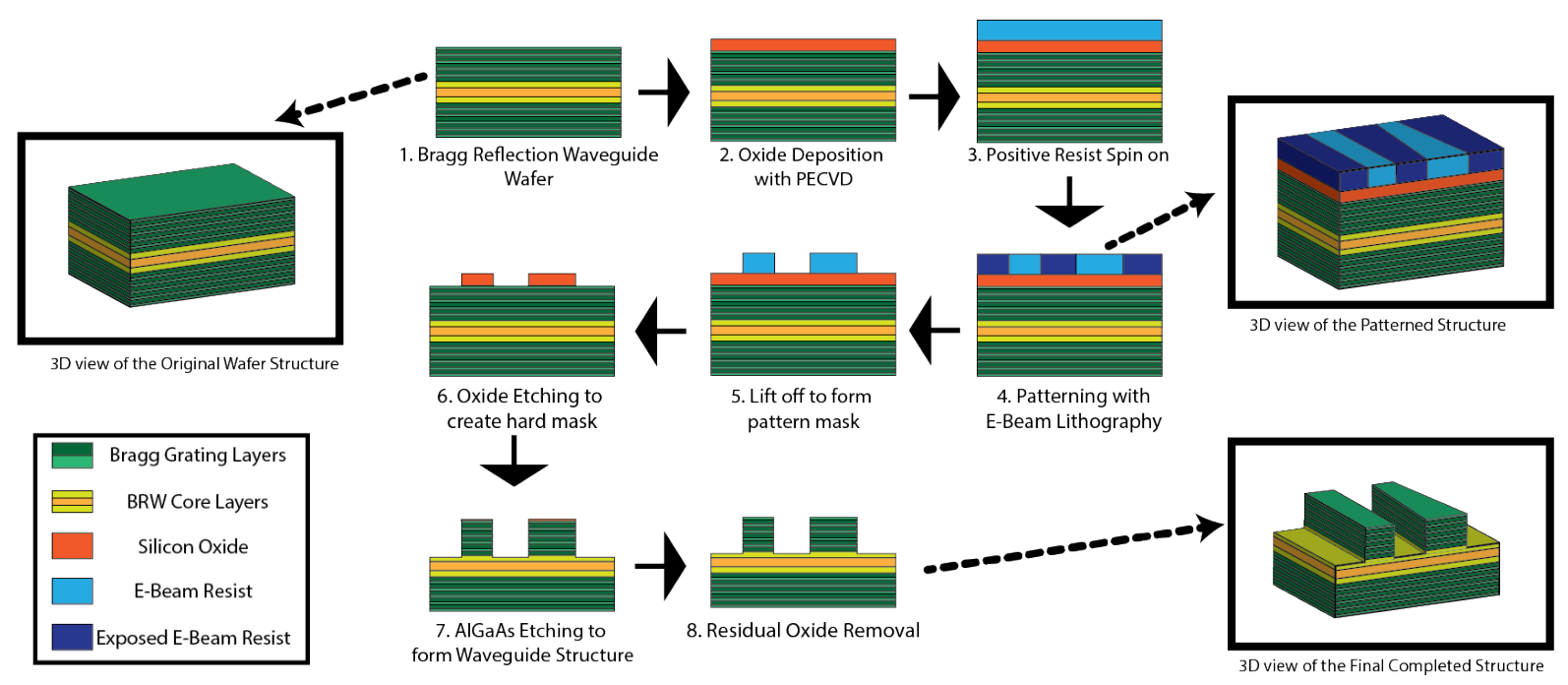

Figure 3.3: Fabrication Flow regarding the tapering implementation on the AlGaAs Structure 


\subsection{Characterization of the Tapered Waveguides}

In this section, tapered waveguides will be characterized the phasematching bandwidth through SHG and DFG. To understand the bandwidth of the optical parametric device, there are two different bandwidths of a device that will need to be characterized. The first one is the pump bandwidth - the pump bandwidth $B W_{\text {pump }}$ in which the pump is phasematched and is able to facilitate nonlinear interaction. The second one is the signal bandwidth $B W_{\text {signal }}$ - the bandwidth of the signal in which, given a pump wavelength, the signal can be phasematched and facilitate the idler generation. To characterize the above mentioned bandwidths of a device, $\chi^{2}$ interactions will be required to probe the nonlinear effect bandwidth. There are two different types of characterizations that will allow us to characterize the two different bandwidth, $B W_{\text {pump }}$ and $B W_{\text {signal }}$ independently. They are second harmonic characterization, which can characterize the $B W_{\text {pump }}$, and difference frequency characterization, which can characterize both the $B W_{\text {pump }}$ and $B W_{\text {Signal }}$.

\subsubsection{Pump bandwidth Characterization: Second Harmonic Gen- eration}

Second Harmonic Characterization allows the probing of the pump bandwidth $B W_{\text {pump }}$ through the use of second harmonic generation (SHG). SHG is the generation of second harmonic wavelength with degenerate signal injection into the device. It is given by the following equation:

$$
\omega_{S H}=2 \omega_{\text {signal }}
$$

It will demonstrate the range of second harmonic generation that this device can operate by injecting the signal. In particular, we are interested in the Type-II process which is shown in Table 3.2. This is because Type-II process has a stronger nonlinear conversion. Also, in Type-II DFG, it allows the ease of isolating the signal or the idler through the use of polarization. 
Table 3.2: Different Types of SHG

\begin{tabular}{cc}
\hline \hline Waves & Type-II Phasematching \\
\hline Degenerate Signal/Signal & TE \\
Degenerate Idler/Idler & TM \\
Second Harmonic/Pump & TE \\
\hline
\end{tabular}

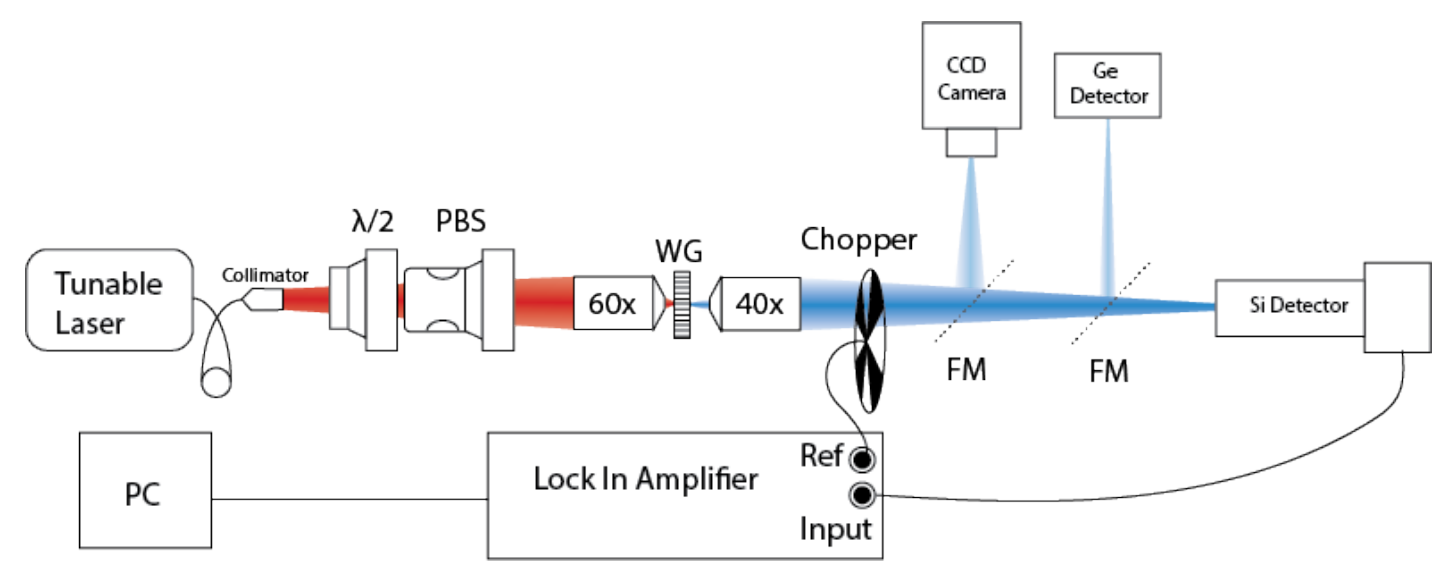

Figure 3.4: SHG Characterization Setup for CW Input

\section{Characterization Setup}

The following experimental setup is used for the second harmonic characterization. A C band tunable laser is used as the signal source for the SHG. The diagram for this setup is outlined in Figure 3.4. The light from the laser is passed through the collimator into the free-space with a fiber. The light is then passed through a polarization controller which is consisted of a half-wave plate and a polarization beam splitter. The half-wave plate will rotate the polarization of the input light such that it can maximally pass through the polarization beam splitter. In this way, the polarization of the light can be confirmed as polarization beam splitter can only pass through on polarization. The polarization controller is set to 45 degree. From this setting, the input signal is dividing half of the power into vertical polarization and half into horizontal polarization. This beam is then coupled into the waveguide with a $60 \mathrm{x}$ lens. With the two independent polarization, the horizontal polarization will be coupled into the TE mode, while the vertical polarization will be coupled into the TM mode. Therefore, it will satisfy the Type-II SHG requirement 
of the polarization in table 3.2. The output beam is coupled out of the waveguide with a 40x objective lens and directed to silicon power detector. This configuration will allow the detection of the second harmonic without influenced by the input signal power as the signal $(1550 \mathrm{~nm})$ is transparent towards the silicon detector while the second harmonic can be detected. The output of the silicon detector is connected to the lock-in amplifier with optical chopper along the beam path to boost the signal-to-noise ratio. As the tunable laser scanned through a range of wavelength, the corresponding detected second harmonic power is recorded through the lock-in amplifier and sent to the computer through a GPIB connection.

\section{SHG Results}

As can be seen from SHG results shown in the Figure 3.5a-3.5f, various tapering degree will result in different SHG bandwidth. The larger the degree, the wider the bandwidth. The corresponding enhancement of the bandwidth can be increased from approximately 1 $\mathrm{nm}$ in Figure 3.5a to beyond $17 \mathrm{~nm}$ in Figure 3.5f. From the theory, it is well known that the SHG bandwidth for a straight waveguide has a sinc function behavior. In Figure 3.5a, although not completely obvious but the slight peaks beside the main lobe are indicating the sinc function nature of the SHG bandwidth. As the tapering degree goes larger, there are separating of the peaks in the spectrum. In particular for Figure 3.5e, the SHG spectrum is closely resembling to the simulation shown in Figure 3.1, with the three prominent peaks in the SHG bandwidth spectrum and over 10nm bandwidth. All the tapered waveguides have shown a higher conversion at the shorter wavelength. This can be attributed to the fact that the waveguides are not lossless and the second harmonics that are generated at the start of the propagation will experience higher loss than the second harmonics generated at the end of the propagation in the waveguide. This trend of lower conversion efficiency that at longer wavelength can be concluded to the fact that the tapering is expanding instead of narrowing along the propagation. This enhancement 


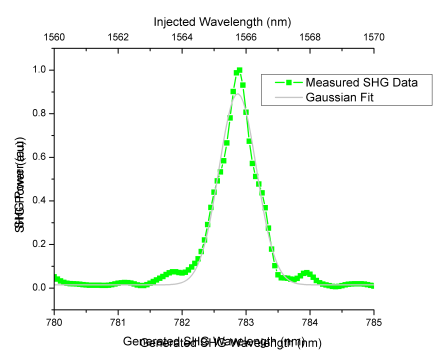

(a)

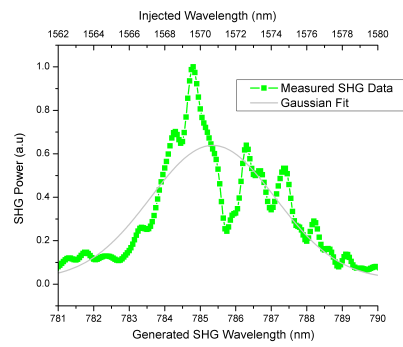

(d)

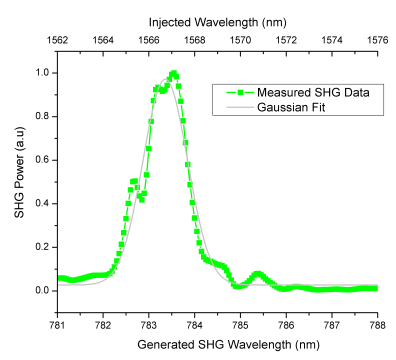

(b)

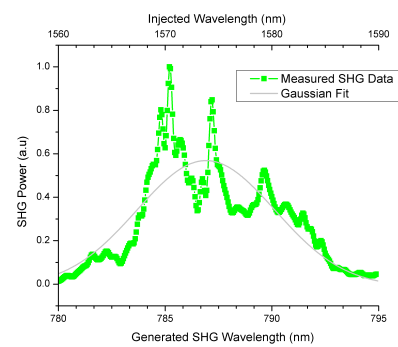

(e)

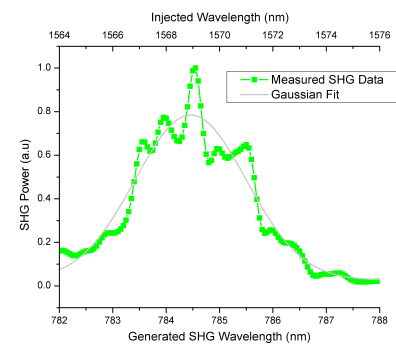

(c)

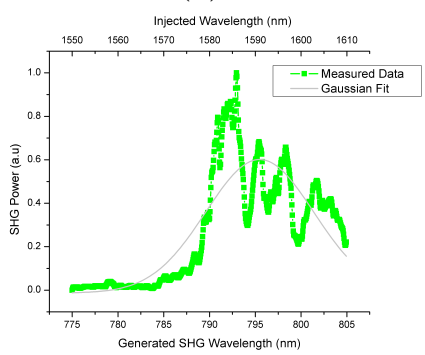

(f)

Figure 3.5: These figures show the measured data resulted from the SHG characterization. The bottom $\mathrm{x}$-axis shows the generated second harmonics wavelength while the top $\mathrm{x}$ axis shows in the injected C-Band pump wavelength. The y-axis shows the linearly normalized measured power of the SHG. (a)-(f) corresponds to the Structure A-F shown in Table 3.1

can allow a wider pump bandwidth and can also allow wider nonlinear interaction.

The result of this enhancement suggests that the $0.5 \mathrm{~nm}$ requirement of pump wavelength accuracy can be relaxed to $17 \mathrm{~nm}$. Although it is at the expense of conversion efficiency, for some situations, this enhancement is particular useful. It can relax the contraints of using a broadband source that is less than $0.5 \mathrm{~nm}$. If the broadband source is larger than $0.5 \mathrm{~nm}$, all the power residing outside of that $0.5 \mathrm{~nm}$ band will not be utilized. By increasing the acceptance bandwidth, a short pulse with broader band source can be more efficienctly utilized. Also, in the case of self-pumping device, where the lasing wavelength and the pump acceptance wavelength is difficult to align, this issue will be greatly benefited from the tapering of the structure and relax the constraint of accurary. It also creates a much more favorable situation for idler conversion tuning, as shown in the next section. 


\subsubsection{Pump bandwidth Characterization: Difference Frequency Generation}

To characterize the pump bandwidth with DFG, the idler power is measured as a function of the pump wavelength. This can be achieved by maintaining a specific signal wavelength while detuning the pump wavelength from the maximally efficient point. Through this method, it can demonstrate the range of detuning of the pump while achieving phasematching condition.

\section{Characterization Setup}

The experimental setup for the pump bandwidth characterization through DFG is shown in Figure 3.6. The pump for the DFG is generated by Continuous wave Ti:Sapphire $780 \mathrm{~nm}$ source while the signal is generated by continuous wave C-band source. The CW pump will be launched into the free space from the laser. The pump, indicated by the blue beam in the figure, will be directed into a polarization controller, which is consisted of a half wave plate and a polarization beam splitter. After the polarization controller has converted the input pump into horizontal polarization, it will be passed through a dichroic mirror at which the pump will combine with the signal. As for the signal path indicated by the red beam in the figure, it will be launched into the free space from the collimator. After passing through a polarization controller designed for the $\mathrm{C}$ band wavelength, it will also be guided to the dichroic mirror at which the signal will be reflected and combined with the pump. The combined beam will be coupled into the waveguide with a 100x lens, and collected out of the waveguide by a 40x lens. The collected beam will then be coupled into a fiber and guided into an OSA to measure the spectrum of the generated idler. Once the idler is detected, the spectrum will be measured while tuning the pump wavelength. This will allow the measurement of the idler power at different pump wavelength in order to characterize the pump acceptance bandwidth. 


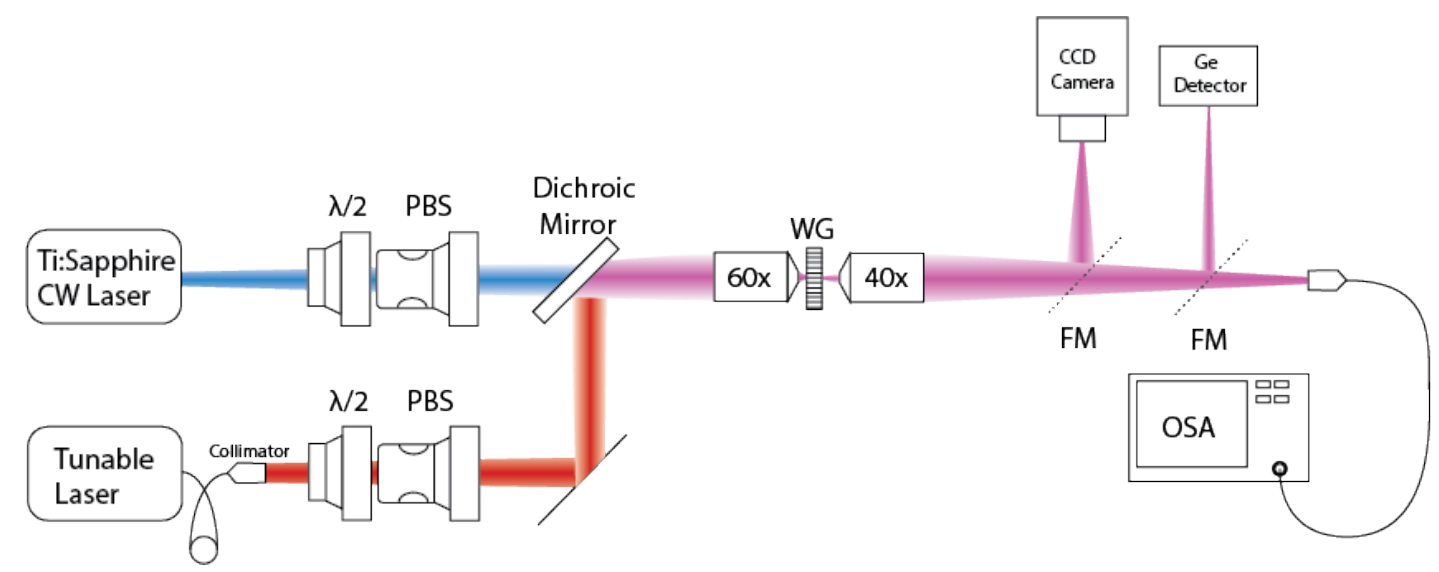

Figure 3.6: DFG Characterization Setup for CW Input

\section{Idler Generation Results}

Figure 3.7a-3.7f show the generated idler power at different pump wavelength. Due to the limited tunability of the Ti:Sapphire CW laser, a finer resolution is not able to be obtained. As it can be seen from the result, it shows reasonable resemblance (in both wavelength and shapes) to its bandwidth obtained from the SHG curve. This suggests that the increase in tapering degree has the effect of increasing the acceptance pump bandwidth for the DFG as well. However, the enhancement is not in well agreement for the waveguide with larger tapering. The limiting and smaller bandwidth from the Waveguide D-F are likely resulted from the lower conversion efficiency. As a result, the generated idler is very close to the noise floor of the OSA and caused the detection difficult.

In Figure 3.8, the estimated bandwidths for SHG and Idler are shown. The bandwidth is extracted from the data with a Gaussian peak fitting. From the plot, there is a strong deviance for the device which has the largest degree of tapering. Its measured bandwidth is about $17 \mathrm{~nm}$ for SHG. Although that waveguide has demonstrated a superior phasematching bandwidth for the SHG, its weak conversion ratio specific to this sample is preventing a proper measurement in the DFG setting. As a result, the resultant bandwidth for the pump detuning is significantly smaller - at $3.8 \mathrm{~nm}$ in comparison to 


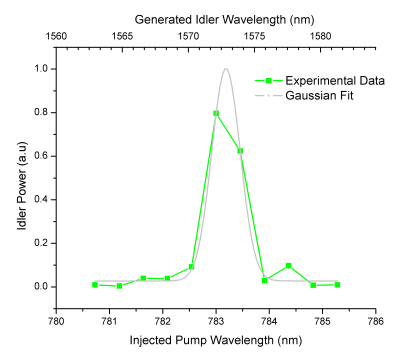

(a)

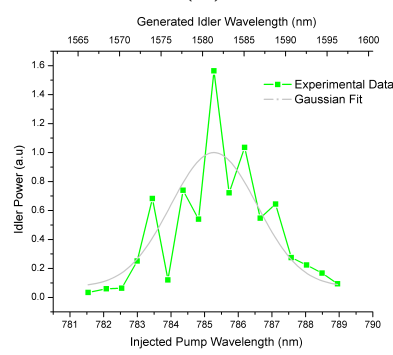

(d)

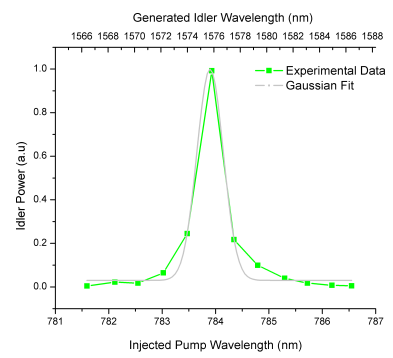

(b)

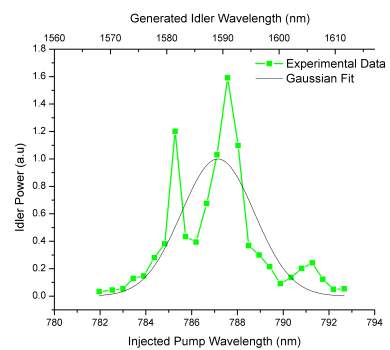

(e)

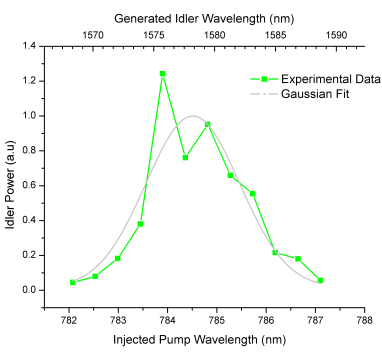

(c)

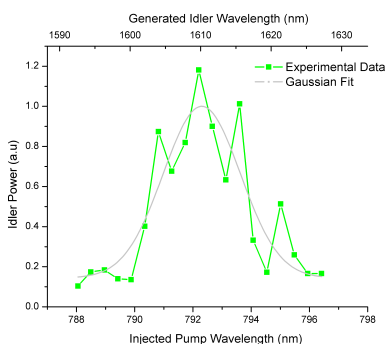

(f)

Figure 3.7: These figures show the generation of idler in power and wavelength in response to detuning of the pump. The bandwidth range is increased from $1 \mathrm{~nm}$ to $4 \mathrm{~nm}$.

17nm. Nonetheless, a larger tapering degree has demonstrated an improved capacity of the idler generation wavelength by detuning the pump wavelength. This enhancement of acceptance bandwidth allows the device to facilitate the operation with a pulse source, which has a larger bandwidth and higher peak power and can enhance the nonlinear conversion further, as well as increasing the tuning range of the idler generation. This will result in a more relax requirement of phasematching. Especially in the situation where the input signal does not have much tunability due to other requirements but the pump can be easily tuned through temperature (such as self-pumping BRW structure), this enhancement of bandwidth will be suitable and can promote the feasibility of these types of application.

\subsubsection{Signal Bandwidth Characterization}

Difference frequency generation enables the probing of the signal bandwidth given a fixed pump wavelength. By injecting a specific pump wavelength which lies within the 


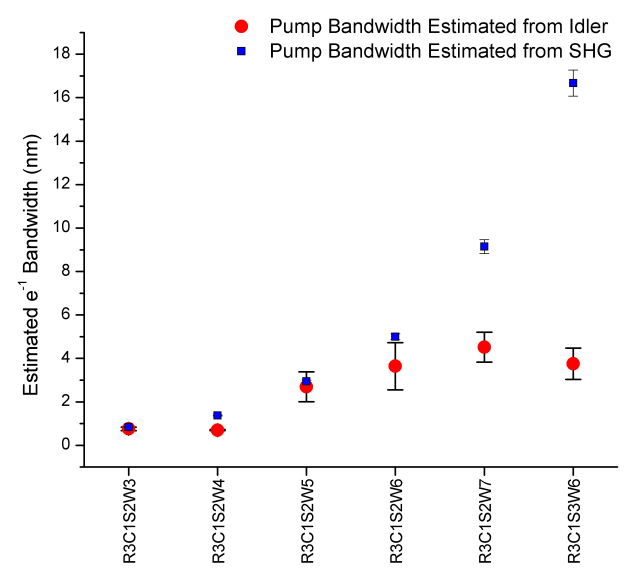

Figure 3.8: Estimated Bandwidth from the measured data of second harmonic generation and Idler generation

pump phasematching bandwidth (close to the degenerate position), there is a range of signal wavelength where it can facilitate the difference frequency generation. The signal bandwidth will enable us to quantify the resulting optical gain bandwidth as well as the range of wavelength conversion. A broad uniform bandwidth is very important for wavelength conversion in the DWDM for the optical telecommunication network. BRW has previously been theoretically predicted to have a flat-band behavior of 100nm FWHM conversion bandwidth. In the section, the signal bandwidth will be characterized through the use of DFG experiment with the pump wavelength at degenerate position.

The bandwidth of the device can be probed by using the setup shown in Figure 3.6.In this experiment, the pump wavelength is fixed at the degenerate position. The signal source which is the CW C-band tunable laser will sweep the wavelength across its operational range. With the pump and the signal coupled into the waveguide with a 100x objective lens, the nonlinear process of DFG can be facilitated The output of the waveguide is coupled into a fiber and guided into an OSA. The wavelength and the power of the input signal as well as the generated idler power is extracted from the OSA.

In the Figure 3.9a, a measured spectrum of the DFG is shown. The input signal is at $1550 \mathrm{~nm}$ while the generated idler is at $1558.9 \mathrm{~nm}$. The waveguide being tested is a 
simple straight waveguide. The conversion efficiency is at $-43 \mathrm{~dB}$. However, this extracted value is modulated by the Fabry Perot effect on the idler and the signal. Figure 3.9b shows the resultant conversion efficiency over a range of signal wavelength. The line indicates the degenerate point of the structure. The half-width half maximum of the phasematching bandwidth of the structure is approximately $50 \mathrm{~nm}$. As a result, the overall FWHM of the phasematching bandwidth is about 100nm. The flat band behavior of the BRW demonstrated over here is important as it proves a wide operation regime for the wavelength conversion in DWDM. The larger the bandwidth, the higher the utilization efficiency as more wavelength can be used within the same device. It also signifies the gain bandwidth, as the conversion efficiency is directly proportional to the gain. It is more superior than the fiber and PPLN where one has a non-flat band behavior and the other one has a 70nm conversion bandwidth.

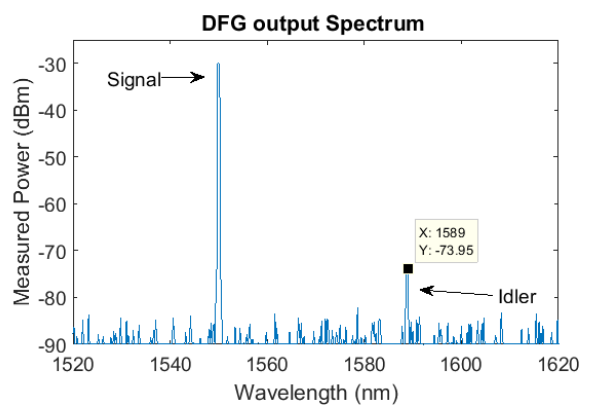

(a)

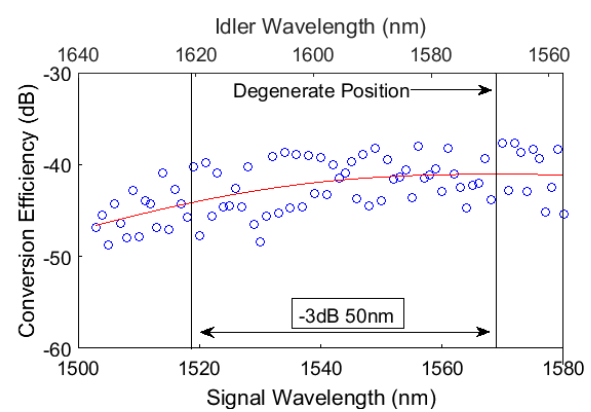

(b)

Figure 3.9: (a) Output spectrum of the DFG with a straight waveguide. (b) Wavelength Conversion showing a peak conversion efficiency of $-37 \mathrm{~dB}$ and 100nm FWHM bandwidth.

\subsection{Summary of the BRW bandwidth and its en- hanced bandwidth design}

BRW design has a relatively narrow acceptance pump bandwidth, which is at around 0.5nm. With a bandwidth enhanced design, the acceptance pump bandwidth can be increased to $17 \mathrm{~nm}$. This suggests that a shorter pulse source with broadened bandwidth 
can be utilized instead of limited by a pulse source with $0.5 \mathrm{~nm}$ bandwidth. This will have a strong implication in the application of the optical telecommunication. This can allow a shorter pulse pump with a higher peak power to facilitate a stronger nonlinear conversion for the optical parametric gain or the wavelength conversion. It is noted that the bandwidth is enhanced at the expense of the conversion efficiency. However, further simulation is required to understand the trade-off among the bandwidth enhancement, usage of the short pulse and the lower conversion efficiency. Nonetheless, the wider acceptance bandwidth will be able to facilitate the tunability of the wavelength conversion. It also improves the feasibility of the self pump device, as it can reduce the stringent requirement of coinciding the lasing wavelength and the phasematching position by broadening the acceptance pump bandwidth. 


\section{Chapter 4}

\section{Parametric Generation in BRW structure}

Parametric Generation is the generation of the wavelength through the nonlinear parametric interaction. It can be used in two important applications that are particularly useful in optical telecommunication and NIR generation. They are optical parametric amplifier, and wavelength converter. It has been demonstrated in PPLN and silicon photonics that, by utilizing nonlinearity provided by the material system, parametric gain can be achieved. Previously, there has not been exploration regarding the optical gain in BRW. In this section, the optical parametric gain regarding to BRW parametric

devices from the simulation as well as experimental characterization will be discussed. A generation of NIR wavelength conversion from 1600nm to $2160 \mathrm{~nm}$, which has not been demonstrated before in the BRW, will also be discussed and demonstrated.

\subsection{Optical Parametric Gain}

Optical parametric gain is resulted from the process of DFG with strong nonlinear interaction. This device are electrically passive - it is optically pumped to facilitate the parametric process. During this DFG process, the energy of the pump is nonlinearly 
absorbed into a parametric energy level, where it is stimulated downconverted into a signal photon and idler photon. When there is a strong nonlinear conversion, there will be a multitude of down conversion from pump to signal and idler. This behavior can be seen from Figure 2.3 which shows the basic behavior of the signal and idler under nondepleting pump assumption. From this model, the parametric gain behavior is clearly seen. However, this model is simple and cannot represent the OPA behavior in the more complex scenario. Nonlinear Schrodinger Equation Solver will be very crucial in understanding the behavior of OPA in a different setting. The formulation is outlined in the Chapter 2.4 and the simulation results are presented in the next section.

\subsubsection{NLSE simulation}

A more detailed optical parametric gain effect can be simulated through the use of the NLSE solver. As the NLSE solver incorporated a wide range of the wavelength parameters detailed in the Equation 2.27, it can represent the behavior of the nonlinear interaction quite accurately. For the operation with pulse system, where the high peak power is leveraged to promote a strong nonlinear conversion, the effect accompanied by the pulse usage such as temporal walk off due to GVM, temporal broadening due to GVD, as well $\chi^{3}$ effects due to high peak power must be taken into consideration. As a result, NLSE is used to simulate the nonlinear behavior with different operation regime. The parameters of the waveguide is detailed in Table 4.1.

\section{Continuous Wave Simulation}

For the simulation assuming a continuous wave operation, we can effectively create a temporal function describing the pulse to be almost square wave by using the following approximation:

$$
A_{\sigma}(t)=A(\tanh (s *(t+P W / 2))-\tanh (s *(t-P W / 2)))
$$




\begin{tabular}{cccccc}
\hline \hline NLSE Parameters & Symbols & Pump & Signal & Idler & Units \\
\hline Wavelength & $\lambda$ & 775 & 1550 & 1550 & $\mathrm{~nm}$ \\
Linear Loss & $\alpha$ & 5.0 & 2.0 & 2.0 & $\mathrm{~cm}^{-1}$ \\
Propagation Constant & $\beta_{1}$ & $2.55 \mathrm{E} 7$ & $1.27 \mathrm{E} 7$ & $1.27 \mathrm{E} 7$ & $\mathrm{~m}^{-1}$ \\
GV & $v_{g}$ & $7.35 \mathrm{E}+7$ & $8.99 \mathrm{E}+7$ & $8.99 \mathrm{E}+7$ & $\mathrm{~m} / \mathrm{s}$ \\
GVD & $\beta_{3}$ & 9.15 & 1.06 & 1.06 & $\mathrm{fs}^{2} / \mu \mathrm{m}$ \\
Effective $\chi^{2}$ Conversion Coefficient & $\kappa$ & & 40 & & $\mathrm{pm} / \mathrm{V}$ \\
Effective Third-Order Area & $A_{\text {eff }}^{(3)}$ & 6.553 & 6.553 & 6.553 & $\mathrm{um}^{2}$ \\
2-photon absorption coefficient & $\alpha_{2}$ & 11.5 & 0.15 & 0.03 & $\mathrm{~cm}^{2} / \mathrm{GW}$ \\
Effective $\chi^{3}$ coefficient & $n_{2}$ & $-1.2 \mathrm{E}-5$ & $3.7 \mathrm{E}-5$ & $3.6 \mathrm{E}-5$ & $\mathrm{~cm}^{2} / \mathrm{GW}$ \\
\hline
\end{tabular}

Table 4.1: Waveguide Parameters Used in NLSE Solver

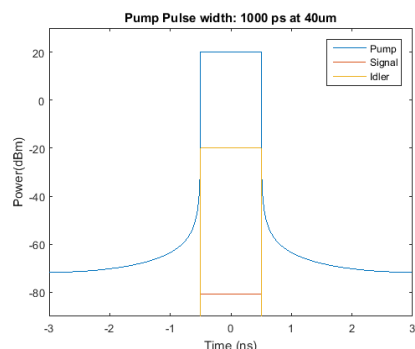

(a)

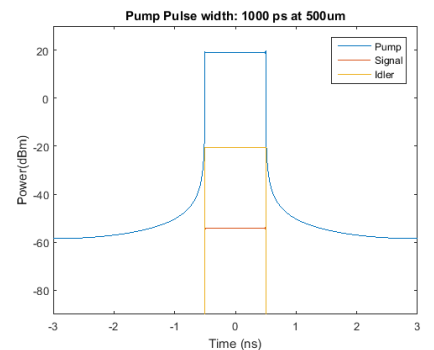

(b)

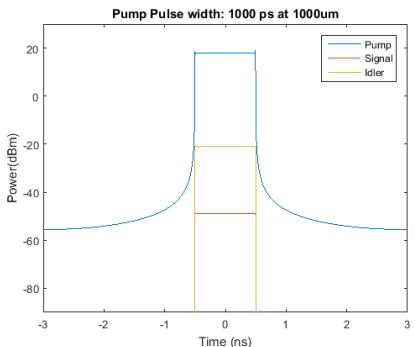

(c)

Figure 4.1: The figures above shows the nonlinear behavior of the continuous wave inside the waveguide at (a) $40 \mu \mathrm{m}$, (b) $500 \mu \mathrm{m}$ and (c)1000 $\mathrm{m}$.

where $\mathrm{A}$ is the amplitude of the field, PW is the specified pulse width, $s$ is a large constant that modifies the function to be more square wave like. To approximate the continuous wave condition, the PW can be set at the order of nanoseconds to alleviate all the GVM and GVD effects. On the other hand, the GVM and GVD coefficients can also be set to zero to turn off the GVD and GVM effects. A sequential snapshot of the Continuous wave operation is shown in Figure 4.1. From the figure 4.1a, it represents the input pump and the input signal are both simultaneously in the waveguide with no idler. This is the situation when the pump and signal coupled in the entrance of the waveguide. As the wave is propagating from $40 \mu \mathrm{m}$ to $1 \mathrm{~mm}$ in Figure $4.1 \mathrm{~b}$ and $4.1 \mathrm{c}$, there is practically no temporal walk off as expected in the CW regime. These figures represent the continuous operation of the optical parametric device. When plotting the power of each wave as a function of propagation distance shown in Figure $4.2 \mathrm{a}$, it can be seen that the increase 


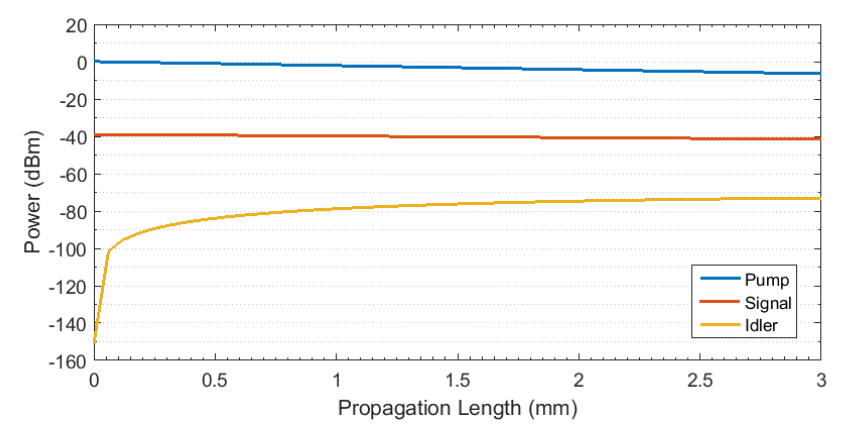

(a)

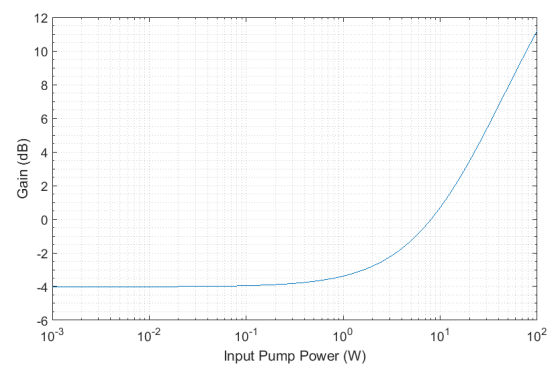

(b)

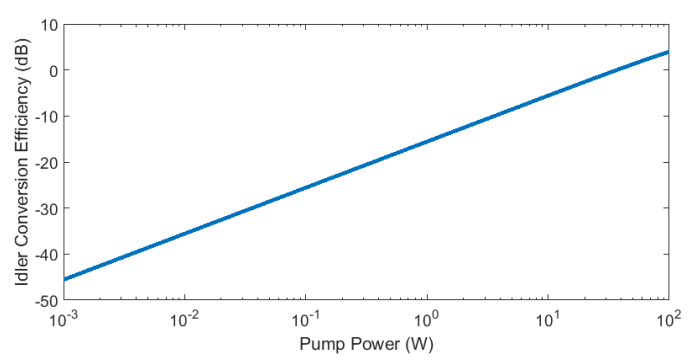

(c)

Figure 4.2: (a) The simulated nonlinear interaction as a function of propagation within the BRW with $1 \mathrm{~mW}$ pump, $0.1 \mu \mathrm{W}$ Signal. (b) Simulated Gain of the device as a function of input pump power. (c) Simulated Idler Conversion efficiency as a function of input pump power.

of the idler power and the reduction of the pump power. The signal power has a general negative slope. This suggests that the parametric gain is not strong enough under the CW scenario to alleviate the linear loss experienced by the signal. To be able to achieve gain in the signal, an analysis is completed to understand the relationship between the gain and the input pump power. The data shown in Figure $4.2 \mathrm{~b}$ is extracted show the gain and input power relationship. To see an appreciable gain level for the signal, the pump power would need to be at the order of $10 \mathrm{~W}$, which is unlikely to be achievable in the CW regime.

\section{Pulse source simulation}

For pulse simulation where both pump and signal input are in pulse and are temporally synchronized, the amplitude of the wave can be correspondingly corrected with the peak 


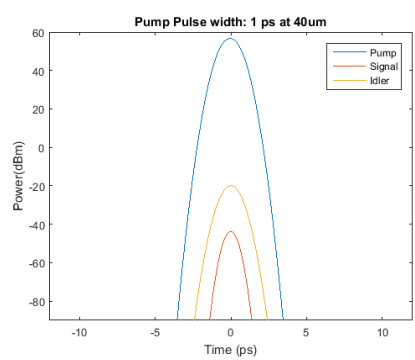

(a)

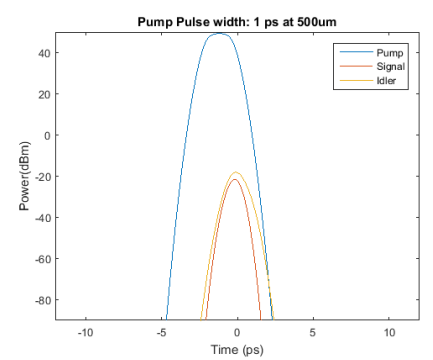

(b)

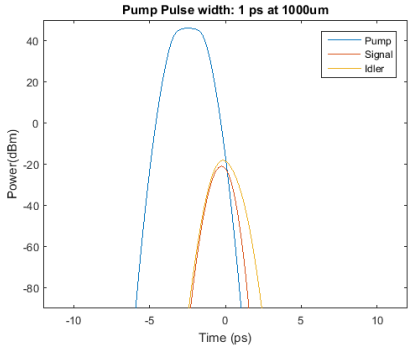

(c)

Figure 4.3: The figures above shows the nonlinear behavior of the continuous wave inside the waveguide at (a) $40 \mu \mathrm{m}$, (b) $500 \mu \mathrm{m}$ and (c) $1000 \mu \mathrm{m}$

power instead of the average power. The peak amplitude can be calculated from the following correlation with the average amplitude if assuming a Gaussian pulse

$$
A_{\text {pulse }}=A_{\text {Average power }} * \sqrt{\frac{0.94}{\tau_{p} f_{\text {repetition rate }}}}
$$

By turning on the GVD and GVM effects, the temporal walk off phenomena can be clearly seen in the snapshots in Figure 4.3 when the pulse width is about 1ps. This temporal walk off will then pose a limitation on the parametric interaction, as after the pulse has walked off, there is no longer any interaction due to the lack of overlapping. All the waves would only experience loss and can no longer experience any parametric interaction. In the regime when the pulse width is about 1ps, the temporal broadening effect can be clearly seen in the Figure $4.3 \mathrm{c}$, where the peak power is significantly degraded due to the broadening. Therefore, there is a trade off between the enhancement of the peak power and the stronger broadening as well as faster walk off from the shorter pulse.

\section{Pulse-CW source simulation}

As for the pulse-CW source simulation, there are two different scenarios of operations. The first one is pulse pump with CW signal, while the second one is CW pump with pulse signal. In either case, the generated idler will be in pulse. This two pulse-CW setting are particular important for characterization. They allow us to probe the behavior of the 
all-pulse setting when there are no appropriate pulse sources simultaneously available or when the temporal synchronization of the input pulses are not readily available. The pulse-CW settings will allow us to explore the effect of the pulse without the equipment to support all pulse setting. To simulate such scenario, the CW is approximated by the square wave like function with a large pulse width while the pulse is approximated by the Gaussian function with a small pulse width.

For the setting with CW pump with pulse signal, there is practically no walk off as the group velocities between the signal and idler are very similar in value and CW pump will not contribute to any walk off. However, this operation is not useful as a parametric amplifier, as the power of the pump is relatively weak to promote a optical parametric gain to the signal. This setting may be more appropriate for the wavelength conversion as a strong idler conversion can be leveraged from the high peak power of the signal.

As for the setting with the CW signal and pulse pump, there is a walk off as the group velocities between the pump and the idler are very different. These behaviors are demonstrated in Figures 4.3. From the Figure 4.4d, it can be seen that, after the walk off has occurred, it will still leave a trail of idler generation but it will be much weaker. Overall, at the peak of the interaction, there is about a $3 \mathrm{~dB}$ gain. However, due to the CW signal setting, the gain for that pulse is not distinguishable from the background CW. As a result, there is very little gain in average power from this setting. However, at that temporal location, it can have as high as $3 \mathrm{~dB}$ gain.

\subsubsection{Continuous wave characterization}

The simplest way to pump the optical parametric device is to pump it with continuous wave source. Through this method, there will be no temporal walk off and broadening effect as the source is narrow bandwidth. The characterization is completed with the experimental setup shown in Figure 3.6. The pump source is the Ti:Sapphire Continuous wave laser (Coherent MBR110) and the signal source for this case is continuous wave 


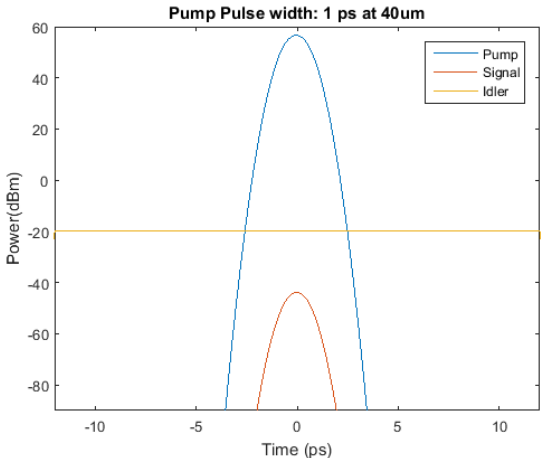

(a)

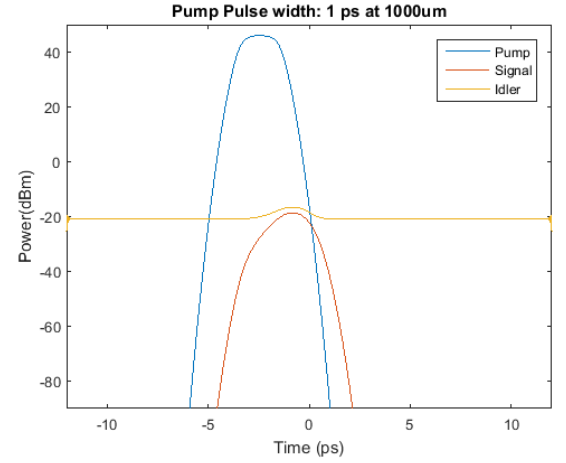

(c)

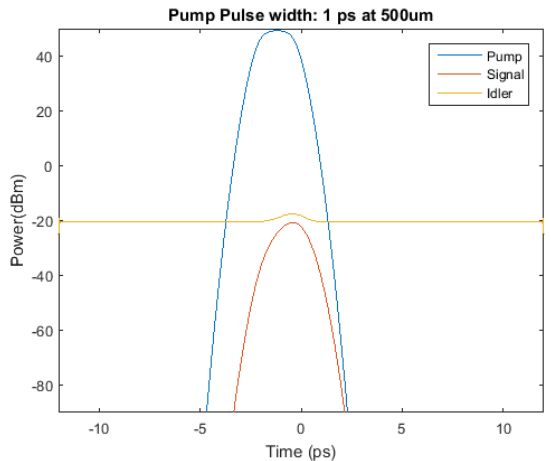

(b)

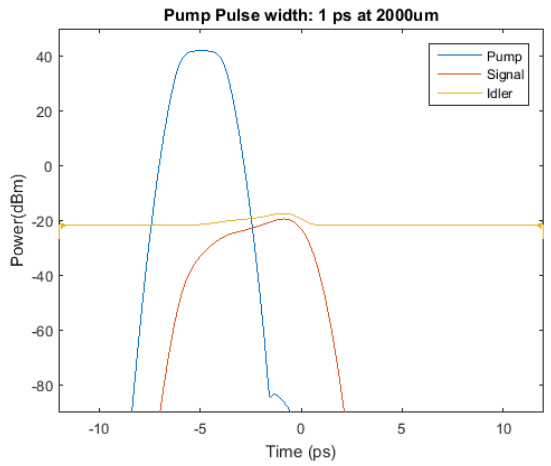

(d)

Figure 4.4: The figures above shows the nonlinear behavior of the continuous wave inside the waveguide at (a) $40 \mu \mathrm{m},(\mathrm{b}) 500 \mu \mathrm{m},(\mathrm{c}) 1000 \mu \mathrm{m}$ and (d) $2000 \mu \mathrm{m}$ 


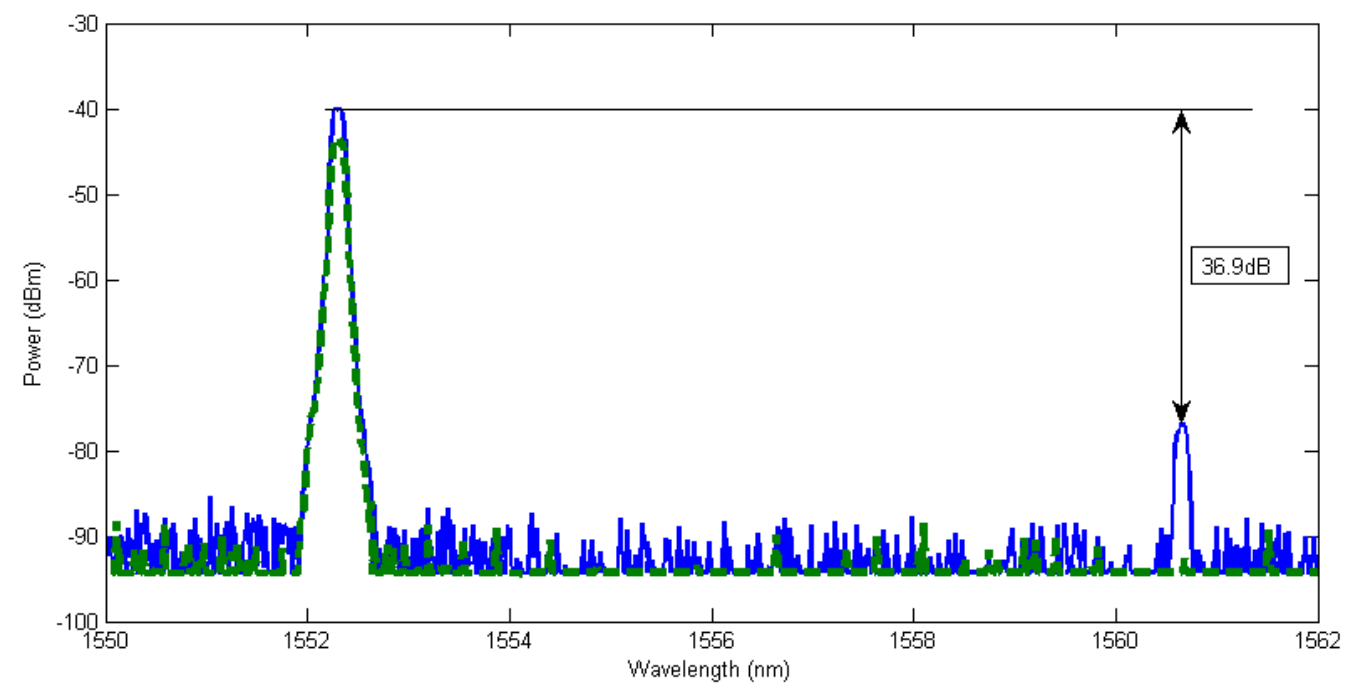

Figure 4.5: The output spectrum of the DFG process. Green is when the pump is off and blue is when the pump is on. The conversion efficiency is $-36.9 \mathrm{~dB}$.

tunable laser (Agilent TL8640). With this setup, the continuous wave optical parametric gain can be performed by measuring the difference of the signal power when the pump is on and off.

By injecting $120 \mathrm{~mW}$ pump power into the waveguide with a $5 \%$ coupled out of the waveguide and $3 \mathrm{~mW}$ signal power into the waveguide with a $2 \%$ coupled out of the waveguide, the output signal spectrum of the BRW straight waveguide is shown in Figure 4.5. The idler power is about $36.9 \mathrm{~dB}$ lower than the signal power. The gain can be seen as the difference between the peak of the signal. As measured by the OSA, the gain over here is shown to be about $3.6 \mathrm{~dB}$.

When scanning the signal across a range of wavelength, Fabry Perot effect with a period of around $0.24 \mathrm{~nm}$ can be seen from the Figure 4.6 in both scenario when the pump is on and off. The shift to the Fabry Perot along the wavelength is likely resulted from the modal effective indices modification of the signal due to the thermal expansion and carrier generation from the absorption of the pump power in the waveguide. By comparing the difference between the signal power with the pump on and pump off shown in Figure 4.6, there is only a oscillator behavior within $4 \mathrm{~dB}$ may not conclude a 


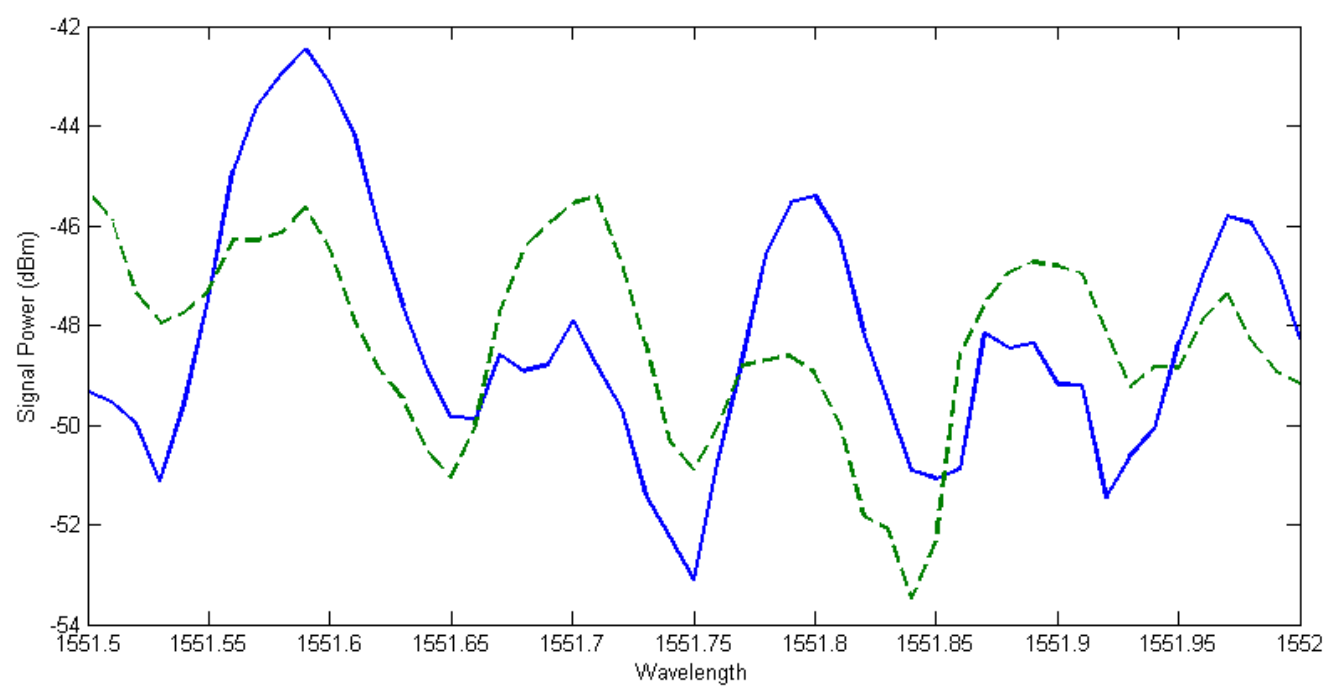

Figure 4.6: Output signal power with the pump on (Blue) and the pump off(Green) at different wavelength. Notice the higher order Fabry Perot effects. Unable to isolate the gain of the signal.

visible gain.

\subsubsection{Pulse-CW characterization}

To leverage the stronger nonlinear conversion provided by the high peak power in the pulse setting, pulse-CW setting is used for the characterization setup. All pulse characterization is not available due to the lack of equipment to accomplish the challenging task. With the Femtosecond Ti:Sapphire Laser as the pump source and the C band tunable laser as the signal source with the characterization setup shown in Figure 4.7, the pulse pump and CW signal characterization can be fulfilled. The pump and the signal will pass through corresponding polarization controller and combine through the use of dichroic mirror. The combined beam is, similar to previous experiments, coupled into the waveguide with a 100x objective lens and coupled out of the waveguide with a 40x objective lens. The output is guided into a fiber where it is connected to a power detector. Gain measurement for the Pulse pump with Continuous wave Signal DFG is challenging due to the dominant power from the CW signal and the low duty cycle of 


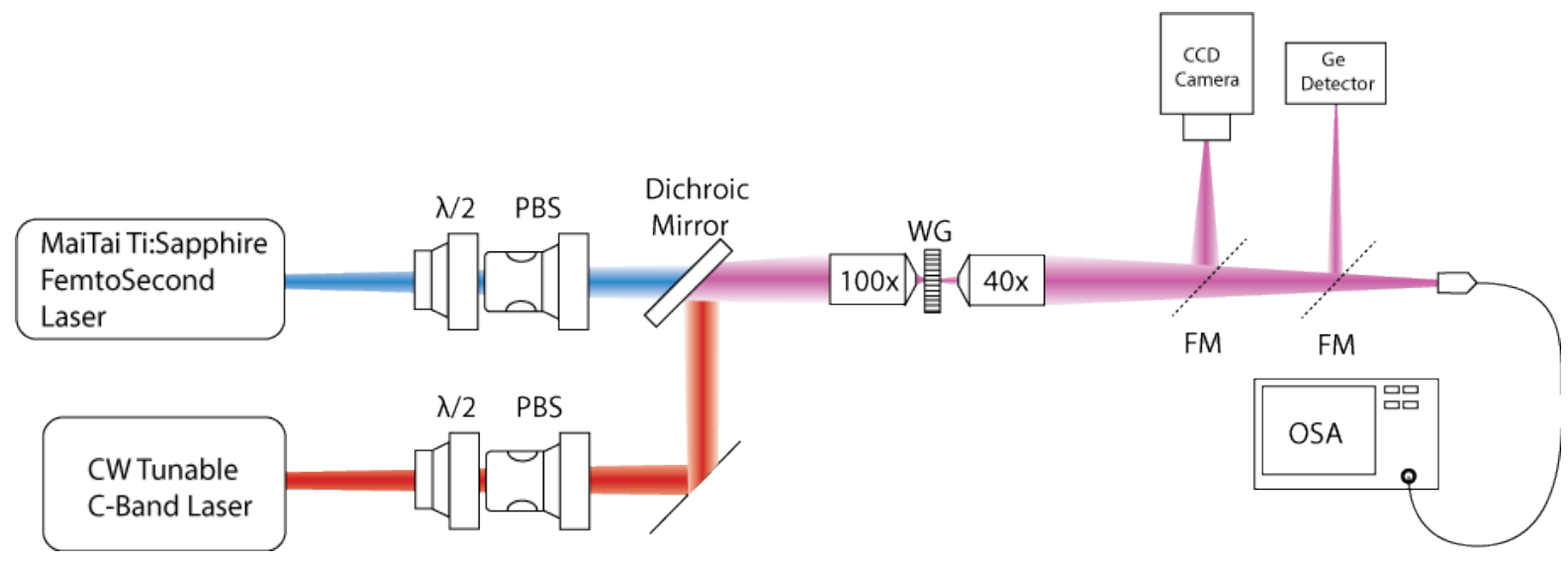

Figure 4.7: Experimental Setup for DFG with the Femtosecond Ti:Sapphire laser as the pump source and the CW Tunable C-Band laser as the signal source.

the pulse. Since the duty cycle of the pulse is as low as $0.0008 \%$ (100fs pulse in $12.5 \mathrm{~ns}$ period), the gain is also acting on that $0.0008 \%$ of signal during the entire cycle. As a result, the gain will not be quite visible in the continuous wave regime. This can be shown in the following experiment. When performing a wavelength scan on the sample, a Fabry Perot pattern can be extracted. The result is shown in Figure 4.8. From the difference between the ratio of the crest and the trough of the Fabry Perot fringes, the propagation loss of the sample can be extracted with the formulation outlined in [67]:

$$
\alpha=\ln \left[\frac{1}{K R}\left(1-\sqrt{1-K^{2}}\right)\right]
$$

and

$$
K=\frac{I_{\max }-I \min }{I_{\max }+I_{\min }}
$$

where $\alpha$ is the propagation loss, $R$ is the reflectivity of the facet, $I_{\max }$ and $I_{\min }$ are the maximum and the minimum values of the Fabry Perot fringes.

From the data, the calculation indicates there is an increase in propagation loss as a function of pump power. The calculated propagation loss is then used in a Fabry Perot and is plotted in the Figure as well. Due to this increase in loss with pump power, the gain measurement with the femtosecond laser would only be visible if the gain can 


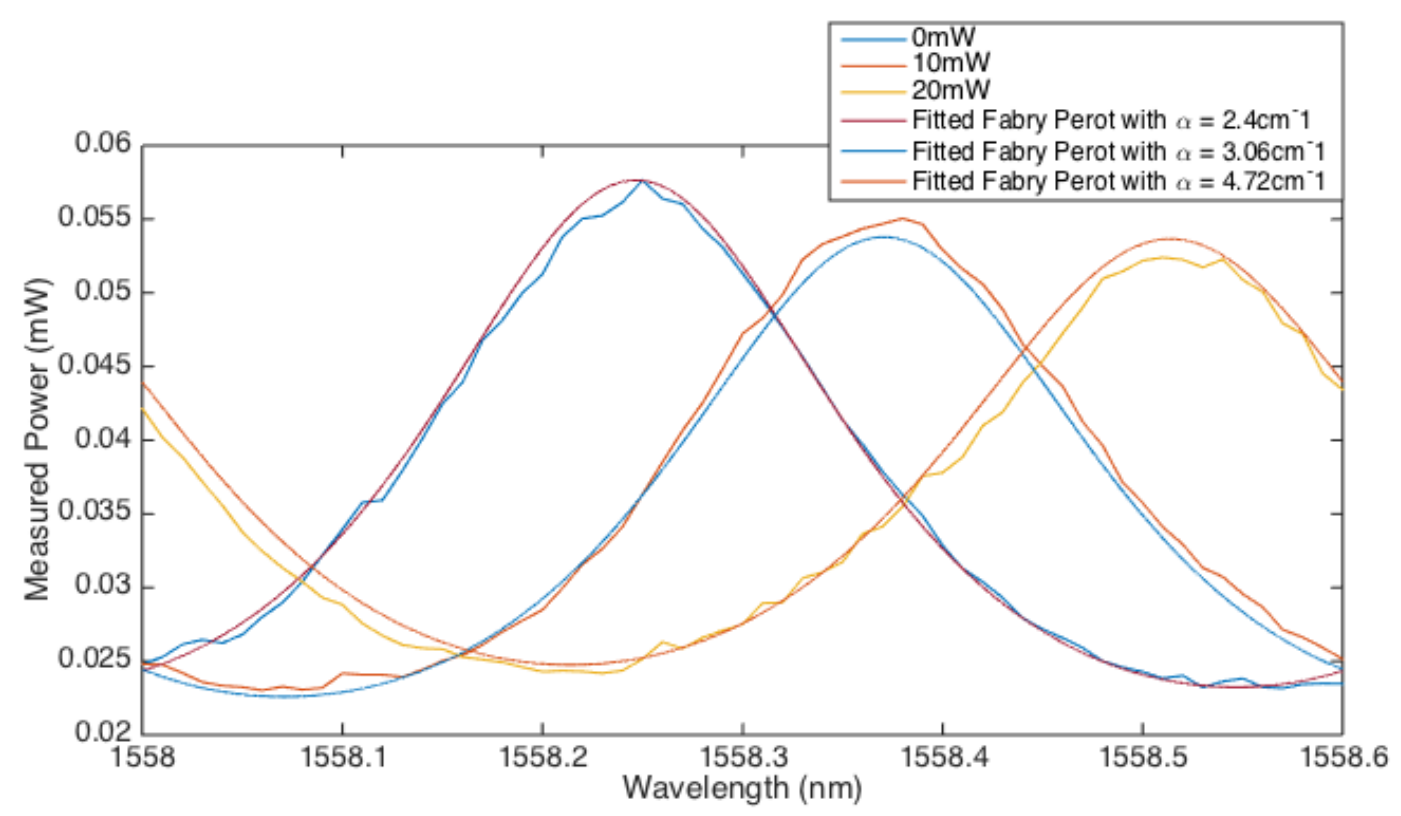

Figure 4.8: Measured Signal power with different Femtosecond pump input power at different wavelength. The data indicates the Fabry Perot Effect.

overcome the increase in loss.

However, it is possible to confirm the pulse behavior is as expected from the NLSE simulation. A fast photodiode is used to detected the temporal behavior of the continuous wave signal with respect to the use of the femtosecond pulse system. The result from the fast photodiode measurement in shown in Figure 4.9. From the simulation, , the full extent of 1 ps pulse behavior is approximately 5ps, which is much less than the rated 14.5ps of the fast photodiode Due to the un-calibrated regime of the fast photodiode as well as timing jitter of the electronics, the measured voltage cannot be used to quantitatively represented the gain of the device. However, it provides a qualitative agreement with the NLSE simulation in Figure 4.4d. In both figures, there are a rise in signal power and a trailing tail following the increase due to the broadening and reduction in peak power of the pump. However, as the pulse width is shorter than the rated specification of the fast photodiode and the electrical system. The raise in the peak cannot be readily accepted as the gain, as it is not calibrated. 


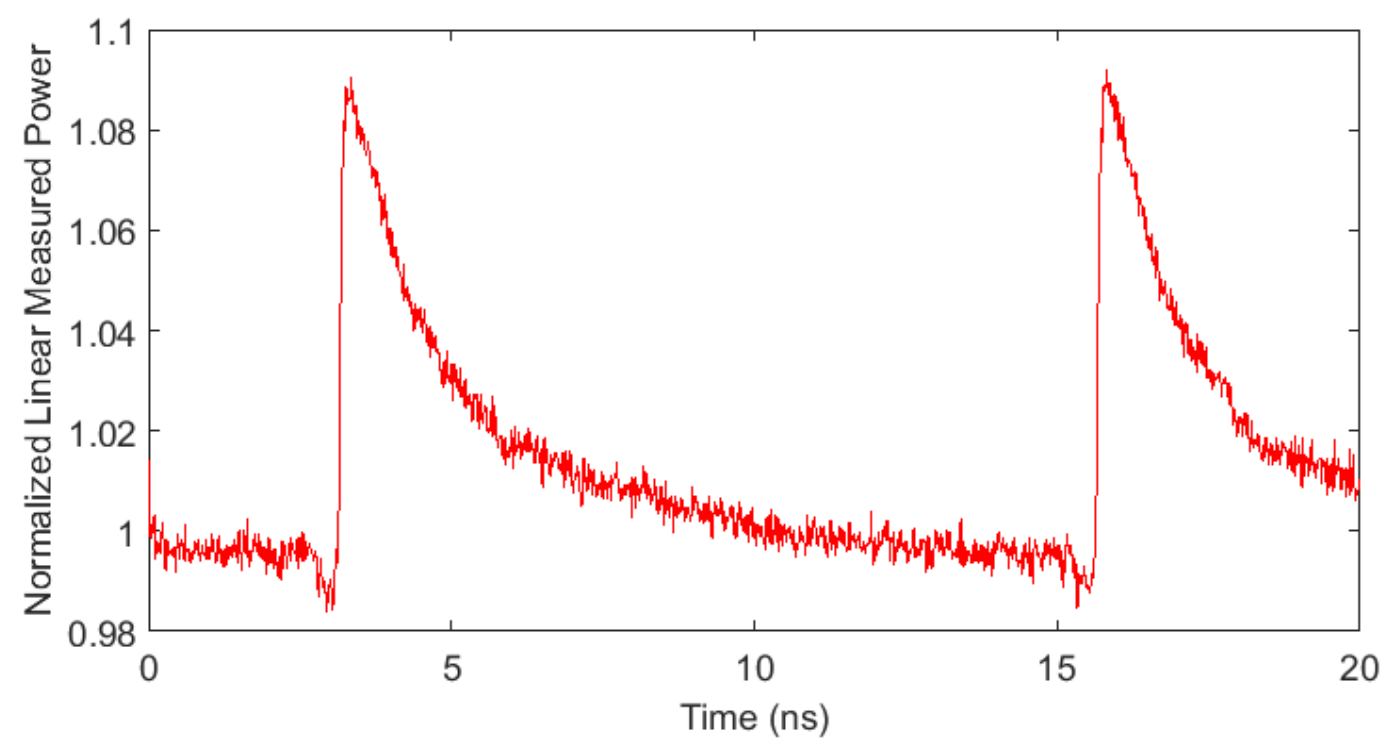

Figure 4.9: Power measurement of the signal power with fast photodiode. The Electrical system is triggered by the sync signal of the Femtosecond laser. The sharp rise and the gradual drop of the signal power is in agreement with the pulse-CW simulation from the NLSE Model

\subsubsection{Wavelength Conversion}

In previous section, it has been demonstrated that the waveguide is able to function as a C-L Band Wavelength Converter when the pump is used at the degenerate wavelength with a peak conversion efficiency at $-37 \mathrm{~dB}$ and bandwidth of $100 \mathrm{~nm}$. This is proven to be important for the telecommunication as a crucial tool for the all optical network. If wavelength beyond L-Band can also be generated, it has further implication in the spectroscopy as well, especially in the $2 \mu \mathrm{m}$ regime. In this section, NIR generation up to 2160 will be attempted.

\section{NIR Generation}

The BRW parametric device can also be used to operate as a NIR wavelength converter up to $2160 \mathrm{~nm}$. This can be achieved by utilizing the pump at non-degenerate position. By utilizing the pump at non-degnerate position, the operation is essentially moving to the left on the tuning curve in Figure 2.2. The regime of operation is demonstrated 


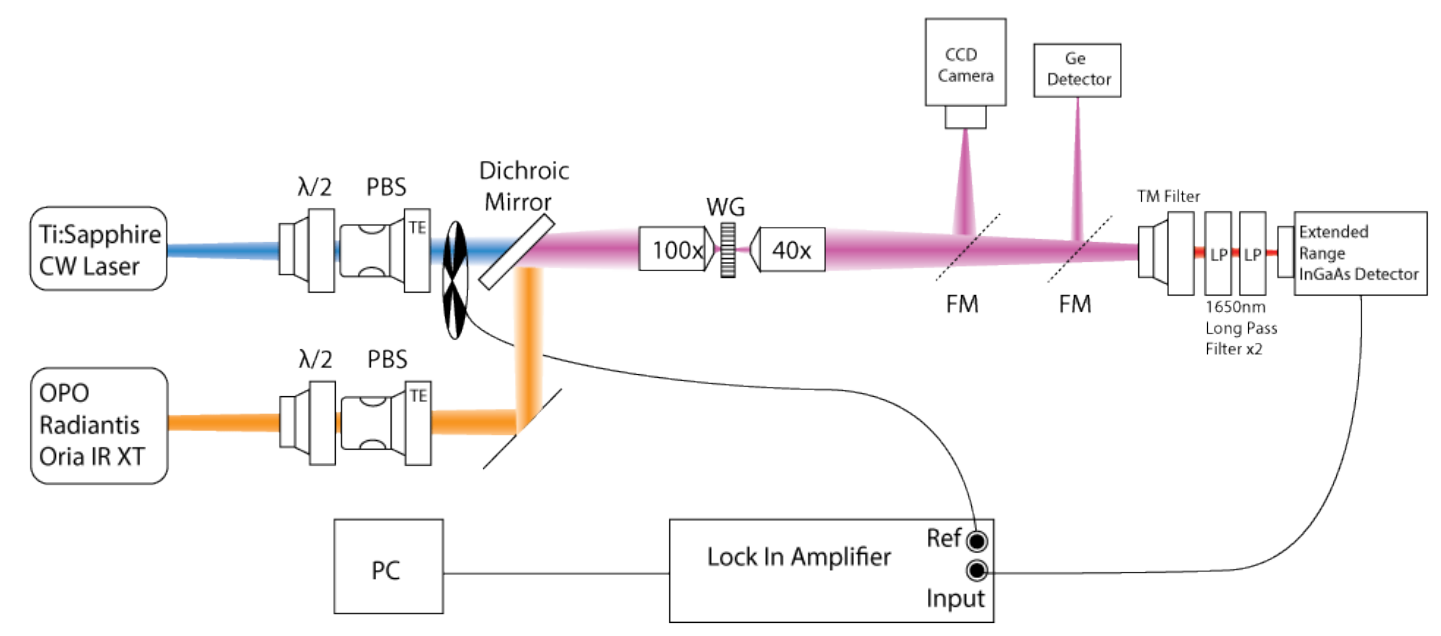

Figure 4.10: NIR Generation Experimental setup with CW Pump and Pulse Signal

through the following experiment shown in Figure 4.10.

The CW Ti:Sapphire 780nm source is used as the pump of the parametric process. In order to generate the beyond $1650 \mathrm{~nm}$, the signal source will have the capability to emit below 1500nm. The Femtosecond OPO source is used as the signal instead of the CW tunable C-Band laser. The pump and the signal is combined together with the use of dichroic mirror and their TE polarization is maintained through the use of half wave plate and polarization. The combined beam is coupled into the waveguide with a 100x objective lens and coupled out with a 40x objective lens. The output is collected by a extended range InGaAs detector which have responsitivity from $1.6 \mu \mathrm{m}$ to $2.6 \mu \mathrm{m}$. However, due to the broadband nature of the OPO, there are some residual power that is detectable by the InGaAs detector. In an effort to suppress the noise, the output beam of the waveguide is passed through a TM polarizer, which will suppress the TE signal power and transmit the TM generated idler power. $1650 \mathrm{~nm}$ long pass filters are also used to minimize any residual from the OPO. The InGaAs detector is connected to the lock-in amplifier. A chopper is then used in the pump path to create a modulated idler power. Given a femtosecond pulse signal wavelength, the wavelength of the pump is then swept 
and the measured idler power is recorded.

The result of the experiment is shown in Figure 4.11. It is capable of generating from $1650 \mathrm{~nm}$ to $2160 \mathrm{~nm}$, by tuning the pump wavelength and the signal wavelength. The peak external DFG normalized conversion efficiency is range from $6.8 \% \mathrm{~W}^{-1} \mathrm{~cm}^{-2}$ at $1400 \mathrm{~nm}$, down to $0.33 \% \mathrm{~W}^{-1} \mathrm{~cm}^{-2}$ at $1150 \mathrm{~nm}$. The internal efficiency would be a lot higher as the external efficiency includes the optical coupling loss from the setup. Moreover, the experiment is only able to generate $2160 \mathrm{~nm}$ due to the limitation of the equipment, as the CW Ti:Sapphire cannot generate below 750nm, and the various components for the signal path does not operate well for wavelength below 1200nm, causing a low power combining after dichroic mirror stage. In comparison with the simulated tuning curve, the experimental tuning curve is in agreement with the simulation. Nonetheless, the experiment demonstrates the possbility of using BRW as a nonlinear medium and is able to phasematched and capable of generating wavelength from $1650 \mathrm{~nm}$ to $2160 \mathrm{~nm}$ through non-degenerate pumping. This has a strong implication in how well the structure can operate at the tuning far away from the designed operation position and showing the wide-tunability of the parametric device.

\subsection{Summary of the parametric generation of the op- tical parametric devices}

Parametric generation is important for both optical network and spectroscopy. In this chapter, simulation behavior is demonstrated when operating with pulse system in the BRW devices and shows the various effects of pulse operation and trade off. In the characterization, parametric gain has been attempted but has not been fully demonstrated in CW and pulse-CW scenario. Nonetheless, wavelength conversion in C-band is shown to have $100 \mathrm{~nm}$ of bandwidth with $-37 \mathrm{~dB}$ conversion efficiency by pumping at degenerate The flat band behavior will be favorable for the application in all-optical network. 


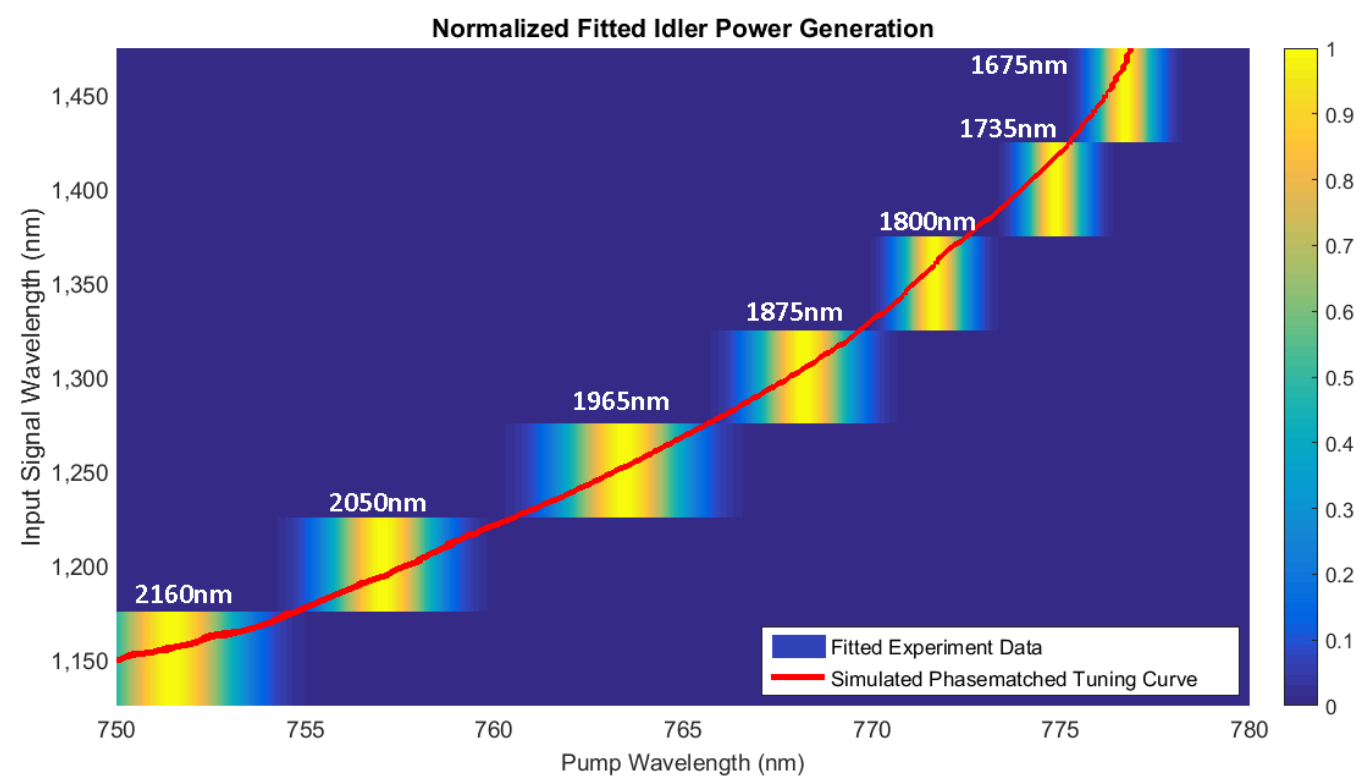

Figure 4.11: Normalized Fitted NIR Generation Data, demonstrating the capability to operate outside of the degenerate regime and perform $\mu \mathrm{m}$ wavelength conversion.

Moreover, NIR generation as far as $2.16 \mu \mathrm{m}$ has been generated through the use of pump at non-degenerate wavelength. This shows the capability of using the optical parametric device as a widely tunable NIR generation and suggests its strong applications in NIR spectroscopy. Although mid-IR generation has not been demonstrated, the NIR spectroscopy has demonstrated the capability of the platform. With a design that facilitate DFG with signal wavelength closer to the pump wavelength, a mid-IR generation can easily be achieved through this platform. 


\section{Chapter 5}

\section{Parametric Generation in BRL}

Bragg reflection waveguide has demonstrated the capability to perform efficient nonlinear conversion. Towards the goal of being a monolithic platform, it is necessarily to demonstrate that the active design in BRW platform can not only function as an active design but also as a nonlinear medium. Bragg Reflection Waveguide Laser Diode (BRL ) has been demonstrated previously. By applying a current through the diode structure, the p-n structure will promote the injection of electrons and holes into the strained quantum well, which will in turn promote the radiative recombination. The quantum well will function as a gain medium for the $780 \mathrm{~nm}$. Along with a cavity provided by the facets of the waveguide, the diode structure will be able to lase along the propagation of the waveguide direction when the Equation 2.24 is satisfied. To achieve lasing in Bragg mode instead of other modes, the overlapping condition provided by Equation 2.25 should also be satisfied as well. With the BRL lasing in the Bragg mode, it should be able to alleviate the low coupling issue and the low pump power within the cavity as discussed previously. However, active structure lasing at $775 \mathrm{~nm}$ with the degeneracy point at around $1550 \mathrm{~nm}$ has yet been reported. To prove the capability of the BRW platform, the BRL not only requires to achieve lasing at $780 \mathrm{~nm}$, and at the same time should be able to perform nonlinear interaction at around 1560nm. Such demonstration of having a BRW structure 
that can electrially self-pumped and perform nonlinear interaction would open the door for monolithic integration in the BRW Platform.

In this section, basic characteristics of laser is first explored and characterized, such that we can quantify the quality of the laser that we are testing. Temperature and current effect of the laser behavior is investigated to extract the laser behavior under different operational parameters. In addition to laser performance, temperature and current injection will result in a change in refractive index of the structure due to thermal effect as well as free carrier generation. An understanding of the phasematching behavior under different laser operation is required and thus characterized as well. With the temperature and current model of the lasing behavior and phasematching modification, a general model can be developed and locate the position where the lasing wavelength and the phasematching position coincide. Once they are coincided, the self-pumped design can be demonstrated.

\subsection{Bragg Reflection Waveguide Laser Diode Design}

A new generation of the BRL has been designed by Nima Zarein. It has the structure shown in Figure 5.1. The top Bragg stack is being p-doped and the bottom Bragg stack is being $\mathrm{n}$-doped. This will form a p-n diode with the BRW structure. At the center of the Bragg Structure, two InAlGaAs compound quantum wells with three barriers $\mathrm{Al}_{0.7} \mathrm{Ga}_{0.3} \mathrm{As}$ are grown in between the core layers. This quantum wells will be able to form a energy trap for the holes and electrons and function as an active region. The design also has a low overlap with the fundamental TIR mode of the 780nm. As a result, bragg mode will have a higher modal gain than the TIR counterpart.

The Quantum well is designed to lase at around 780nm with TE mode, while the Type-II phasematching position is around 786nm. This will provide flexibility to tune the lasing wavelength correpondingly to match with the phasematching and perform 


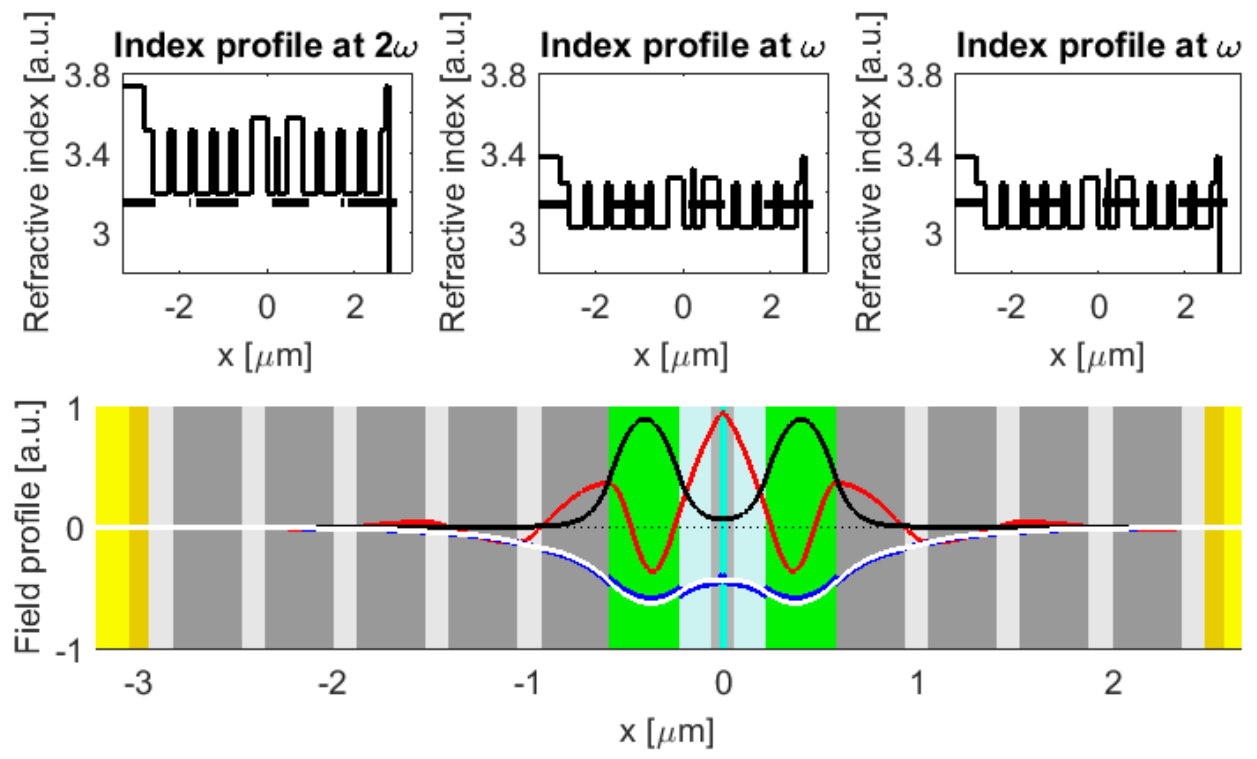

(a)

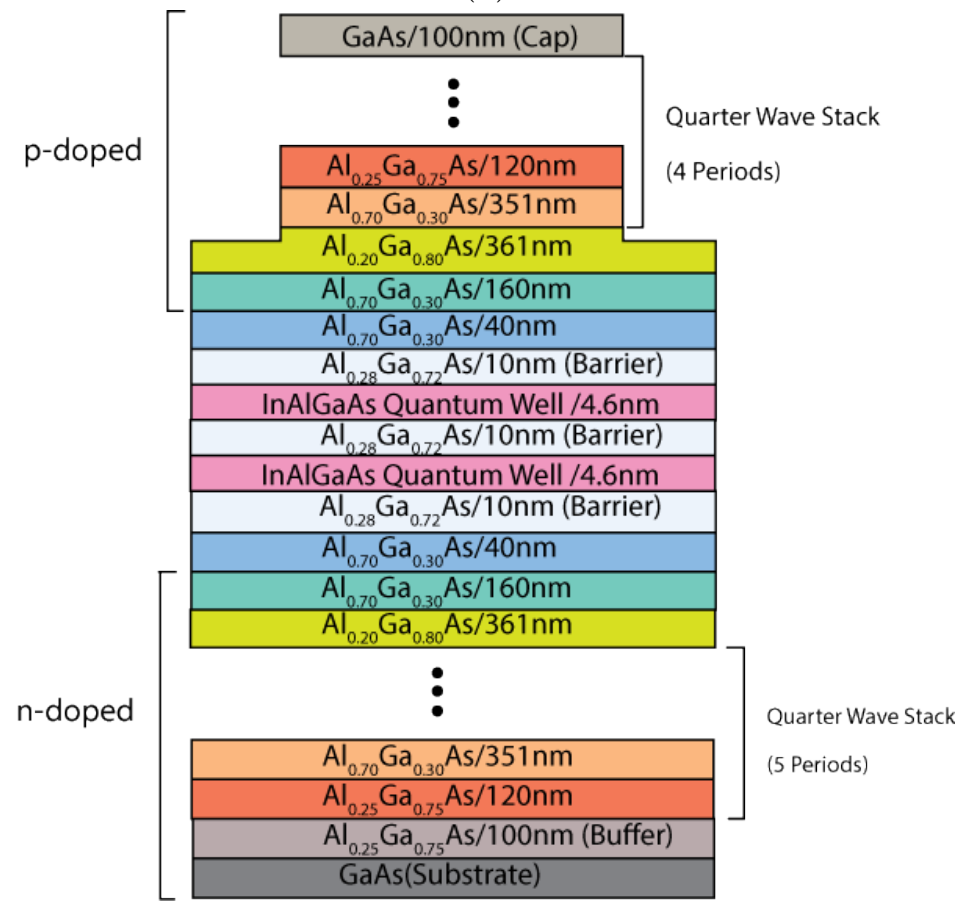

(b)

Figure 5.1: (a) The 1D Mode solver result showing the effective indices of each waves as well. It also shows the field profiles of the pump Bragg TE mode (Red), pump TIR TE Mode (Black), Fundamental TIR TE Mode(White) and Fundamental TIR TM modes. (b) shows the details of the AlGaAs layers for BRL 8 Structure. 


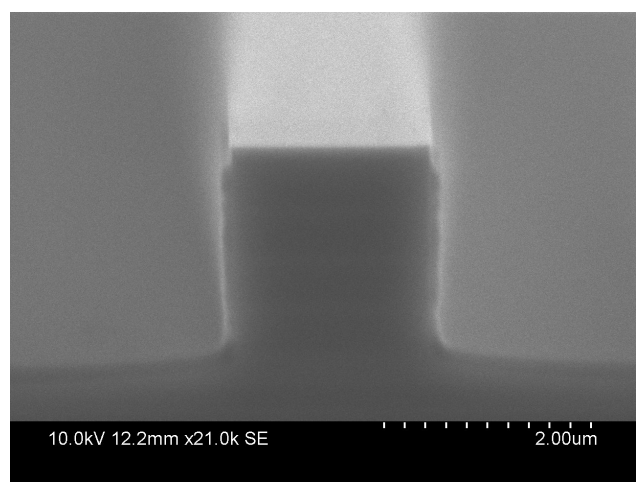

(a)

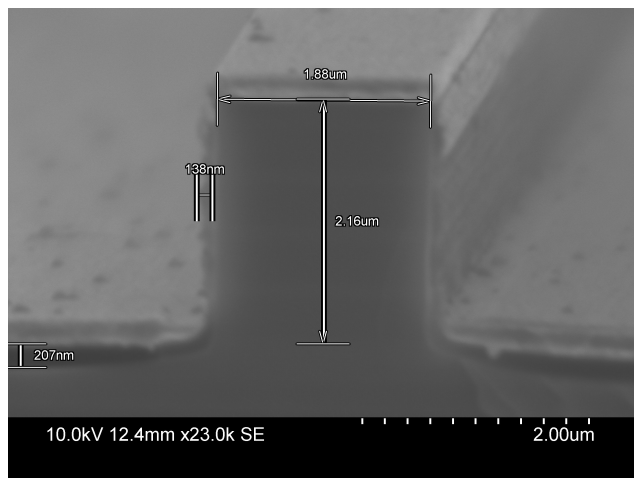

(b)

Figure 5.2: (a) BRL 8 Waveguide after growing of electrical insulation silica layer. (b) BRL 8 Wavelength after Ti and Au contact deposition.

Type-II nonlinear process with TE pump and TE/TM fundamental mode.

The laser are fabricated by Eric Chen, Master student working under Professor Amr Helmy, in University of Toronto. The Scanning Electron Microscope (SEM) images are shown in 5.2. As can be seen the SEM images, there is no much structual anomaly on the waveguide before the contact. However, after the contact disposition, the side wall seems extremely rough. This is due to the non-conformal disposition from the E-beam Evaporation technique.

\subsection{BRL Laser Performance}

To understand the device quality and its ability to achieve self-pumping, a range of laser characterizations are laid out in this section. Basic Laser characterization is completed by putting the laser on a copper block without bonding. The experimental setup is shown in Figure 5.3. The laser diode sample is sitting on a copper block. As the laser sample has a n-contact at the bottom, the copper block will function as the ground of the system. It will also serve as a temperature controlled stage. A electrical probe can be lowered to come into contact with the top electrical contact pad on the surface of the sample. Laser diode current source (Keithley 2510) is used to electrically inject the current into 

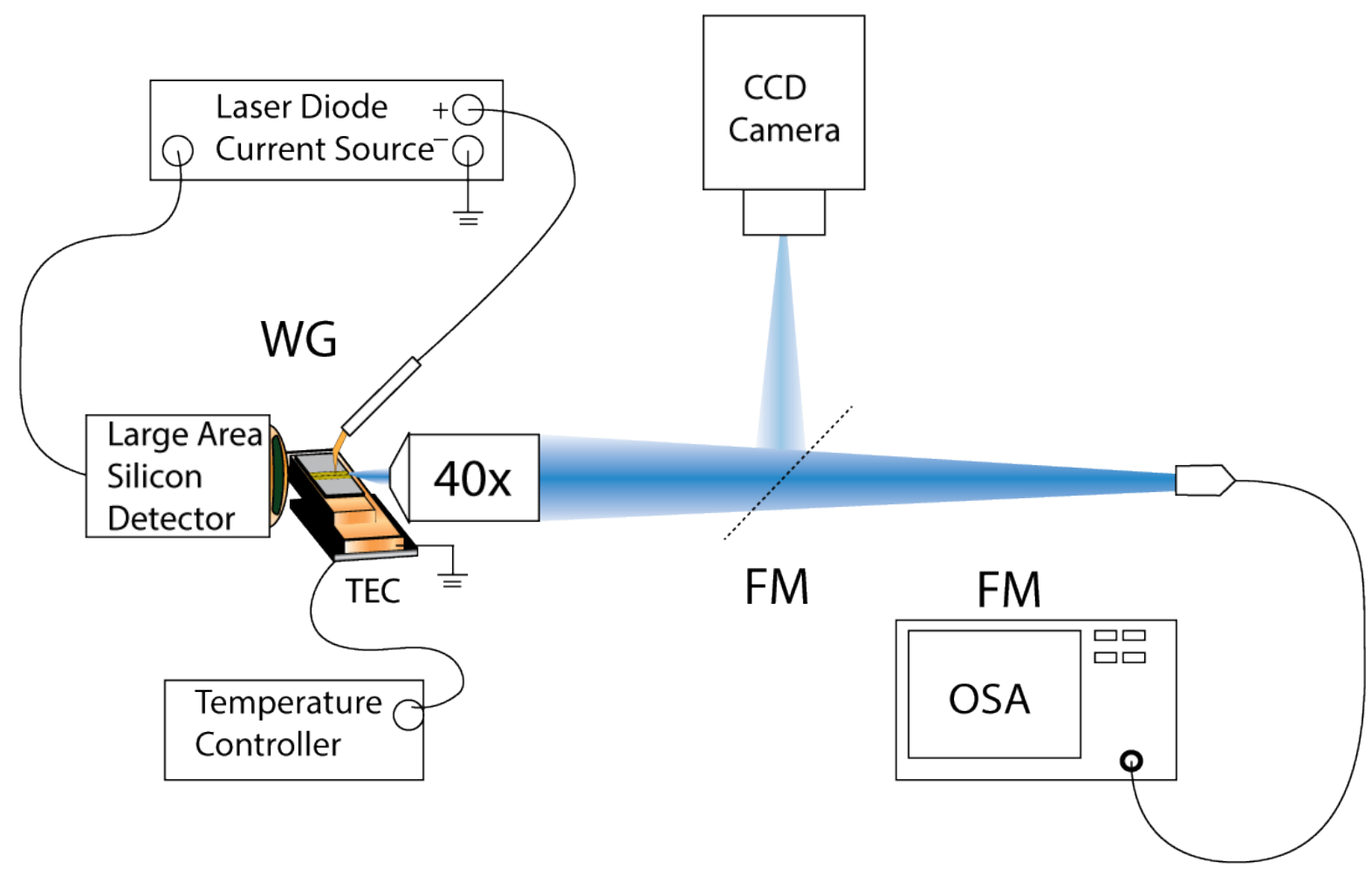

Figure 5.3: Experimental Setup for characterizing laser performance of the laser diode sample.

the sample. Temperature controller (Keithley 2520) is used to control the copper block stage temperature. The output of the laser is directly coupled into a Large Area silicon detector which is used to measure the output power of the laser from one of the facet. The spectrum and the near field profile is obtained through a 40x objective lens coupling out of the other output facet. With this setup, Luminescence-Current-Voltage (LIV) of the laser can be extracted.

The LIV result is shown in Figure 5.4a. In this experiment, it is completed with pulsed current source with $1 \mu$ s pulse width and $20 \mu$ s pulse delay at $15^{0} \mathrm{C}$ on a bonded sample. It displays a typical laser behavior. There is a low power output primarily resulted from the spontaneous recombination until it reaches the threshold current. After the threshold, it has a sharp and almost linear increase in power. It reaches close to $7 \mathrm{~mW}$ at $300 \mathrm{~mA}$. This sample has a dimension of the ridge structure to be $2 \mu \mathrm{m}$ wide and $950 \mu \mathrm{m}$. The threshold current for this sample is $44 \mathrm{~mA}$ and the threshold voltage is $2.4 \mathrm{~V}$. The corresponding 


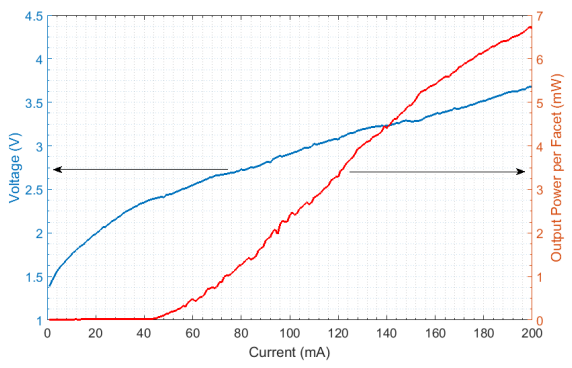

(a)

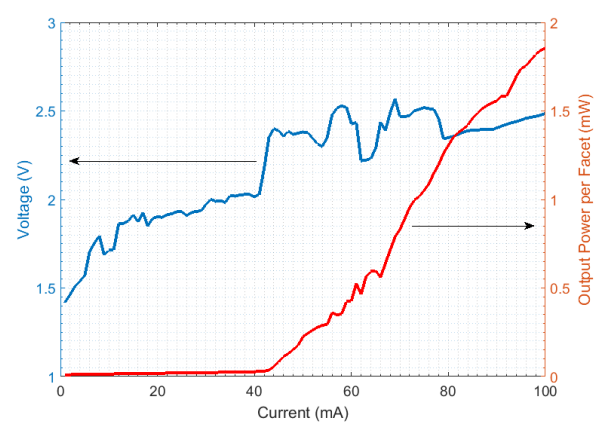

(b)

Figure 5.4: (a) LIV of a bonded Bragg laser diode. Threshold current is $44 \mathrm{~mA}$ and threshold voltage is $2.4 \mathrm{~V}$. (b)

threshold current density is $2263 \mathrm{~A} / \mathrm{cm}^{2}$. It has a external differential quantum efficiency of 0.056 with a dynamic resistance of $10 \Omega$. Compared with the previous generation, there is an increase in the threshold current from the $1700 \mathrm{~A} / \mathrm{cm}^{2}$ and in the dynamic resistance from $5 \Omega$ as well. The low differential quantum efficiency indicates that the device has high propagation loss for the Bragg mode. When operated in DC mode, the LIV is shown in Figure 5.4b. It is able to reaches $1.8 \mathrm{~mW}$ at $100 \mathrm{~mA}$, which is not unreachable in direct current on the unbonded sample. The strong fluctuation in voltage suggests the contact disposition has not been refined yet. There is likely a nonuniform current density passing through the structure, and creating local hot spot and damage the device. The external differential quantum efficiency is lowered from 0.056 in pulse mode down to 0.048 in DC mode.

\subsubsection{Temperature Effect}

Temperature can modify the bandgap of the quantum well, and in return, it will modify the lasing wavelength. This provides a mean to tune the lasing wavelength given a laser structure. It is especially important as the lasing wavelength may not align perfectly with the phaematching wavelength. To explore the lasing behavior with the temperature, the same setup in Figure 5.3 is being utilized. The Pulse mode current source with $1 \mu$ s pulse 


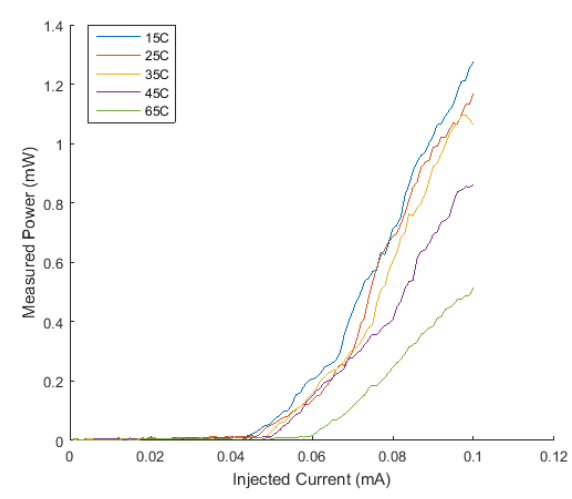

(a)

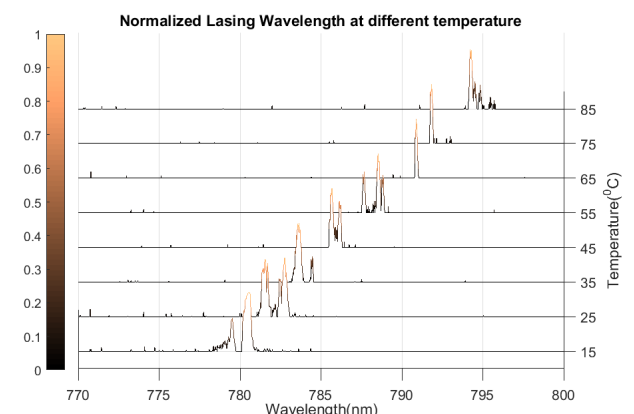

(b)

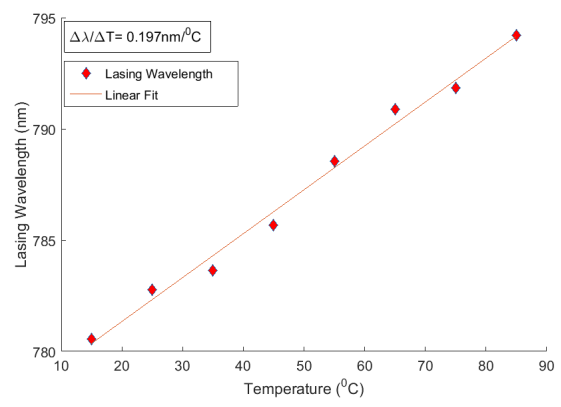

(c)

Figure 5.5: (a) BRL lasing spectra from $15^{0} \mathrm{C}$ to $85^{0} \mathrm{C}$. (b) Extracted linear relationship between the lasing wavelength and the change in temperature. A shift of lasing temperature at around $0.197 \mathrm{~nm} /{ }^{0} \mathrm{C}$

width and $20 \mu$ s pulse delay is used to limit the thermal effect introduced from the current injection. LIV is extracted for each temperature set by the TEC attached to the copper block, and the output spectrum is collected by a fiber and guided to an optical spectrum analyzer.

In Figure 5.5a, the LIV results at different temperature is plotted. It shows a trend of increase in threshold current at higher temperature and the differential efficiency gradually decreases. This can be primary be attributed by the temperature dependent loss such as the Auger recombination. In Figure 5.5b, the spectra of the laser output is shown at different temperature. A linear relationship between the lasing wavelength and the temperature can be extracted from the lasing peak and shown in Figure 5.5c. The coefficient relating the lasing wavelength and the temperature, $\Delta \lambda_{\text {laser }} / \Delta T$ is $+0.197 \mathrm{~nm} /{ }^{0} \mathrm{C}$. 


\subsubsection{Current Effect}

Injection of higher current level will not only allow the more radiative recombination in the quantum well (higher power output), but also significantly increases the temperature of the structure due to the dynamic resistance. To explore the current effect on the lasing spectra while limiting the thermal effect, pulse mode operation of the current source is used with the same setup shown in Figure 5.3 and the spectra of the laser output is obtained at various level of current injection at $20^{\circ} \mathrm{C}$.

In Figure 5.6a, the spectra at different current shows that as current injection level increases, more modes that are longer wavelength are excited. It can be noted that the profile of the lasing spectrum retains from the low current at higher injection current. It can be hypothesized that, even with $1 \mu$ s second of injection, the junction temperature is raised to a high degree. This causes the excitation of other longer wavelength modes instead of the shorter wavelength modes. Therefore, it indicates that, in order to properly operate in the pulse mode, current injection must be just slightly above threshold to retain the narrow bandwidth of the output spectra.

When driving the current in continuous wave (dc), the ohmic heating of the structure resulting from the current injection will cause the junction temperature in a linear behavior. This behavior on the bonded is shown in Figure 5.6b. To investigate the trend of the lasing wavelength in response to heating originated from direction current injection. The result of this behavior is shown in Figure 5.6, where the lasing peak is linearly proportional to the injection current. The shift of the lasing wavelength, $\Delta \lambda_{\text {laser }} / \Delta I$ is $+0.122 \mathrm{~nm} / \mathrm{mA}$. With this value, the raise in temperature of the junction in regard to current can be approximated with the following:

$$
\frac{\Delta T}{\Delta I}=\frac{\Delta \lambda_{\text {laser }}}{\Delta I} *\left(\frac{\Delta \lambda_{\text {laser }}}{\Delta T}\right)^{-1}=0.62^{0} C / m A
$$




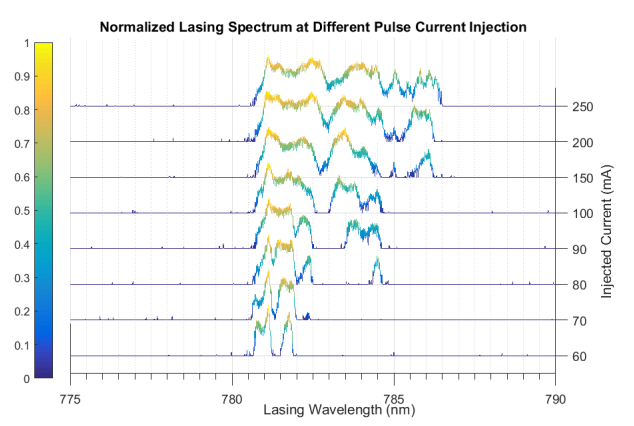

(a)

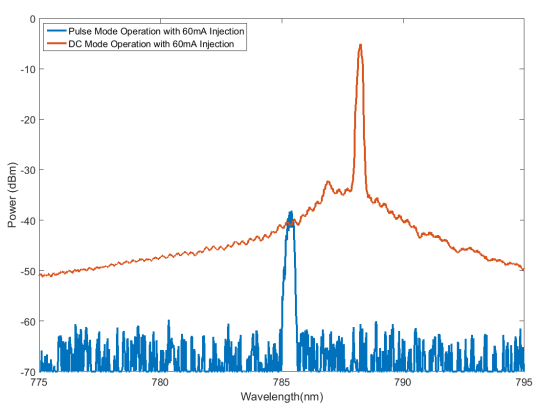

(b)

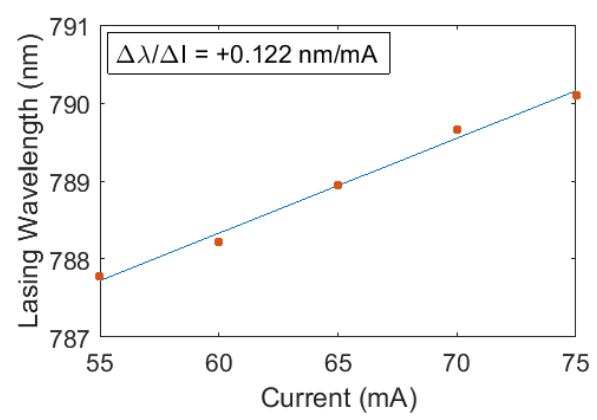

(c)

Figure 5.6: (a) Unbonded $2 \mu \mathrm{m}$ ridge Laser Output spectra at different current level with pulse width at $1 \mu \mathrm{s}$ and pulse delay at $20 \mu \mathrm{s}$.(b) Bonded $2.2 \mu \mathrm{m}$ ridge Laser spectra output at pulse mode and at DC mode. (c) Extracted unbonded $2 \mu \mathrm{m}$ ridge laser output wavelength with respect to DC current injection.

The laser wavelength can be modeled by the following equation:

$$
\Delta \lambda_{\text {laser }}=\frac{\Delta \lambda_{\text {laser }}}{\Delta T} T+\frac{\Delta \lambda_{\text {laser }}}{\Delta I} I
$$

where $\Delta \lambda_{\text {laser }}$ is the change in lasing wavelength, $\mathrm{T}$ is the change in temperature, $\mathrm{I}$ is the injected current.

\subsection{BRL Nonlinear performance}

The nonlinear properties of the BRL structure will be characterized through Type-II SHG process. The experimental setup is shown in Figure. A CW tunable C-band laser is used to emit the pump at the range of $1550 \mathrm{~nm}$ through a collimator. The light is 


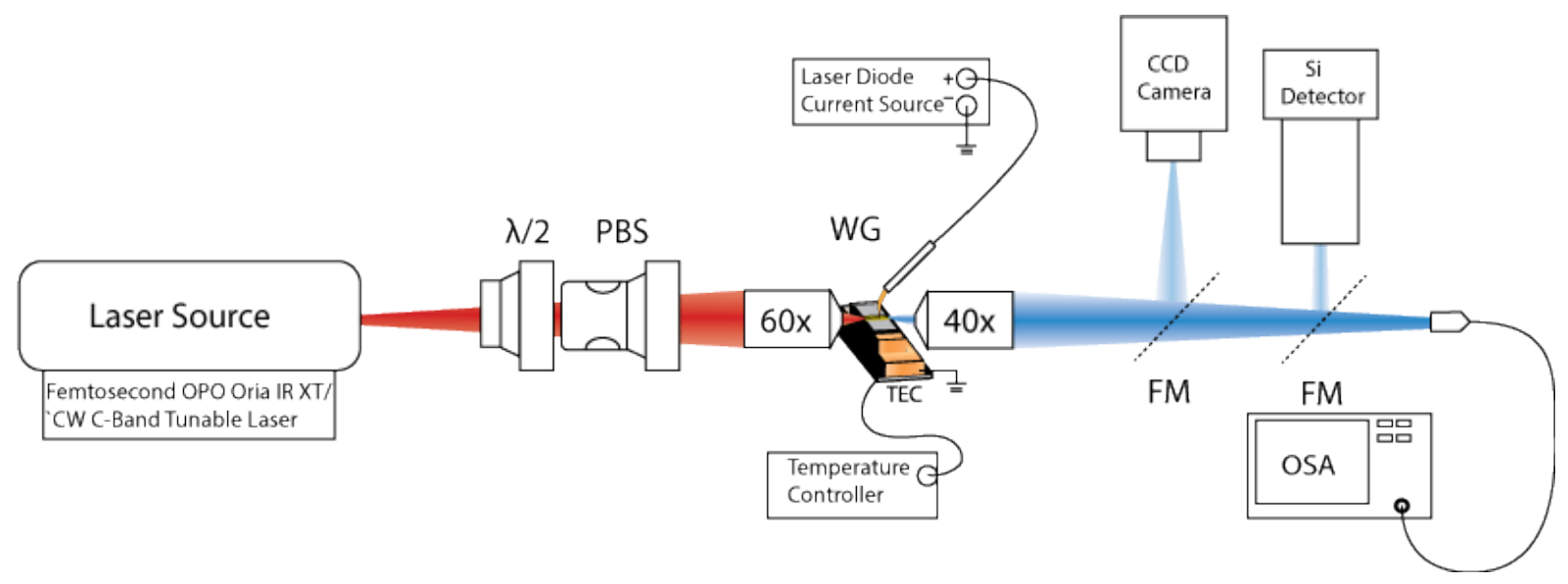

Figure 5.7: Experimental Setup for SHG nonlinear performance characterization with laser diode. CW laser source is used to characterize the laser diode nonlinear conversion efficiency optically. Output of the second harmonic is collected with the Si detector. Femtosecond Laser source is used to characterize the phasematching behavior in response to temperature and current effects. SH output is collected into fiber and guided into an OSA for spectrum analysis.

passed through a half-wave plate and a polarizer beam splitter to turn the polarization into 45 degree (50\% horizontal and 50\%vertical). A $60 \mathrm{x}$ lens is used to couple the light into waveguide. The 45 degree configuration will allow the half of the power to couple into the TE mode with the other half coupled into the TM mode. The output of the waveguide will be coupled by a 40X and directed to a Silicon detector where the 1550 is transparent and only second harmonic can be detected. As the tunable laser sweeps through the wavelength, second harmonic will be generated when the phasematching wavelength is satisfied.

The second harmonic generation with the CW Tunable C-Band Laser is shown in Figure 5.8. As can be seen from the figure, there are three major peaks. From left to right, they are Type-I (TM Second-Harmonics and TE Fundamental), Type-0 (TM SecondHarmonics and TM Fundamental) and Type-II (TE Second-harmonics and TE/TM Fundamental). The input of the C-band pump laser is $16 \mathrm{~mW}$ and the generated Type2 SHG power is $2 \mathrm{nW}$ at $1572 \mathrm{~nm}$. This indicates the phasematching second harmonic for this structure is approximately $786 \mathrm{~nm}$. The normalized conversion efficiency is 


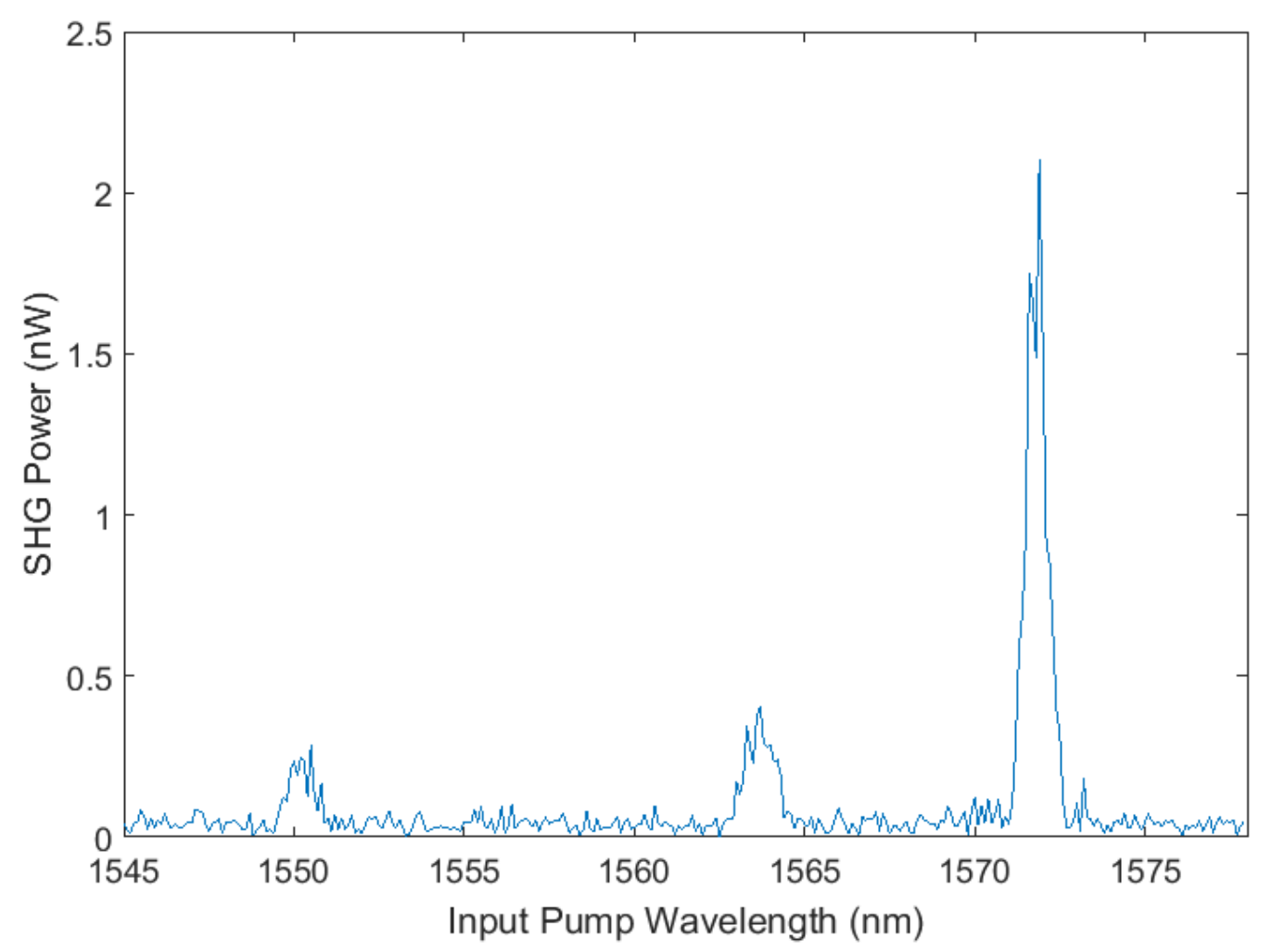

Figure 5.8: SHG Spectrum generated from CW tunable source

$0.087 \% \mathrm{~W}^{-1} \mathrm{~cm}^{-2}$.

\subsubsection{Temperature effect}

Temperature has the effect of changing the refractive index of the structure. At a higher temperature, the refractive index will increase. Therefore, an understanding of how the phasematching position in accordance to the temperature will be required to align with the lasing wavelength. The experimental setup is shown in Figure 5.7. A Femtosecond $1550 \mathrm{~nm}$ OPO is used as a broadband source. The polarization of the pulse is modified to 45 degree through the use of half-wave plate and the polarization for the Type-II phasematching. It is coupled into the sample positioning on a copper block with a $60 \mathrm{x}$ lens. The output of the sample is coupled out with a 40x objective lens and is guided into an optical spectrum analyzer through coupling back into a fiber. The TEC installed 


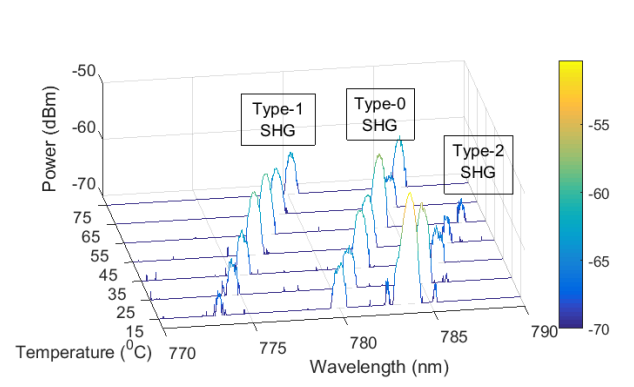

(a)

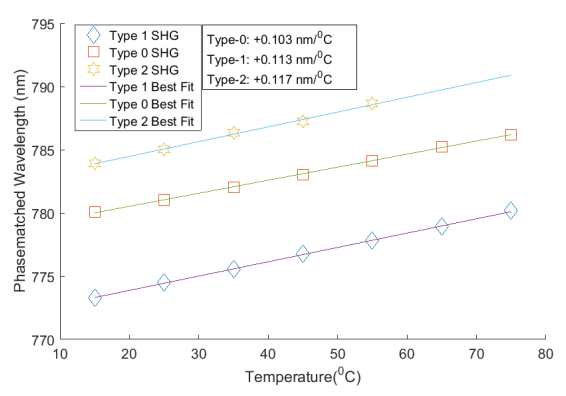

(b)

Figure 5.9: (a) Optically pumped SHG Spectra of the laser diode sample at different temperature. (b) The linear fit and the trend of the shift in phasematching wavelength in response to different temperature.

to the copper block will be able to control the temperature of the copper block and the sample.

The measured spectra is shown as a waterfall plot in Figure 5.9a. Since the femtosecond pump is a broadband source, it can excite all three types of SHG without any Fabry Perot effect. As a result, the spectra of the phasematching can be easily visualized. By extracting the peak of the SHG from the spectra at different temperature, a linear relationship between the phasematching position and the temperature of the sample can be created and it is shown in Figure 5.9b.

$$
\Delta \lambda_{\text {Thermal_Type:2,1,0}}(T)=\frac{\Delta \lambda_{\text {Type: } 2,1,0}}{\Delta T} T
$$

where the $\Delta \lambda_{\text {Thermal_Type:2,1,0 }}(T)$ is the shift in phasematching wavelength for Type 2 $(1,0)$. The net shift of the Type-II phasematching, $\Delta \lambda_{\text {Type } 2} / \Delta T$, is $+0.117 \mathrm{~nm} /{ }^{0} \mathrm{C}$, while for Type-I, $\Delta \lambda_{\text {Type } 1} / \Delta T$, and Type- $0, \Delta \lambda_{\text {Type } 0} / \Delta T$, are $+0.113 \mathrm{~nm} /{ }^{0} \mathrm{C}$ and $+0.103 \mathrm{~nm} /{ }^{0} C$ respectively. As there is a difference of about $0.1 \mathrm{~nm}$ between the temperature shift of the lasing wavelength and phasematching wavelength, it indicates that the difference can be leveraged to align the lasing wavelength and the phasematching wavelength. 


\subsubsection{Current Effect}

Injection of current introduces free carriers into the structure. The free carriers will reduce the refractive index of GaAs[68]. On the other hand, it is also capable of heating up the structure due to ohmic heating. The raise in temperature also give rise to increase in refractive index. To investigate the two folded effect of current injection on the phasematching, the following experiment is set up as shown in Figure 5.7. It is essentially the same as the experiment from the previous section. Instead of modifying the temperature of the stage, different direct current level from the electrical probe on the p-contact is injected, and the output SHG spectra is monitored with the OSA.

The measured spectra at different direct current is shown in Figure 5.10a. From the different representation in Figure 5.10b and Figure 5.10c, it can be shown that the Type1 and Type-0 Phasematching has similar parabolic red shift trend from the injection of the current. However, as for Type-2, the phasematching position blue shifts initially and then red shifts. The lasing wavelength can also be seen on the spectra at and above $55 \mathrm{~mA}$. The lasing wavelength is $788 \mathrm{~nm}$ and is at a much longer wavelength than the pulse mode operation from Figure 5.6a that we have demonstrated before. This is the result of the high junction temperature due to the direct current and unbonded structure. Therefore, this shift in phasematching wavelength can be concluded as a combined effect of free carriers and temperature. As we know that the thermal effect has a linear relationship with the wavelength in Equation 5.3 and, from previous calculation,

the raise in temperature as a function of current, $\frac{\Delta T}{\Delta I}$ has been obtained, these can be represented in the following equations (focusing only on Type-2):

$$
\begin{aligned}
\Delta \lambda_{\text {Type } 2}(I, T) & =\Delta \lambda_{I_{-} \text {Type } 2}(I)+\Delta \lambda_{\text {Thermal_Type } 2}(T) \\
& =\Delta \lambda_{e^{-} \_ \text {Type } 2}(I)+\Delta \lambda_{I_{\text {induced_Thermal_Type2 }}(I)+\lambda_{\text {Thermal_Type } 2}(T)} \\
& =\Delta \lambda_{e^{-} \_ \text {Type } 2}(I)+\frac{\Delta \lambda_{\text {Type } 2}}{\Delta T} *\left[\frac{\Delta T}{\Delta I} I+T\right]
\end{aligned}
$$




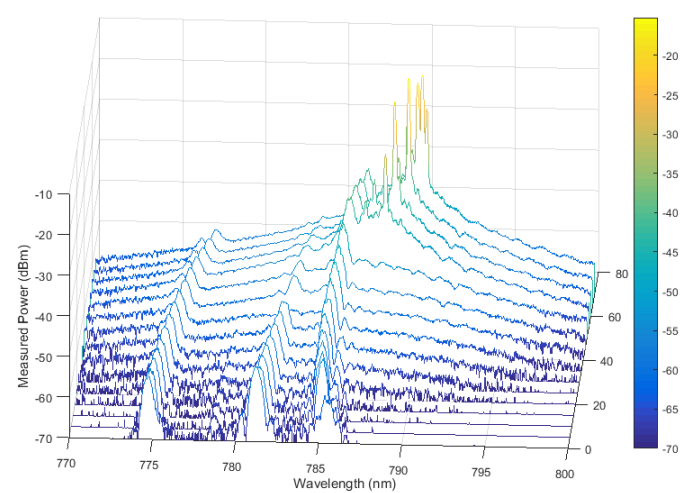

(a)

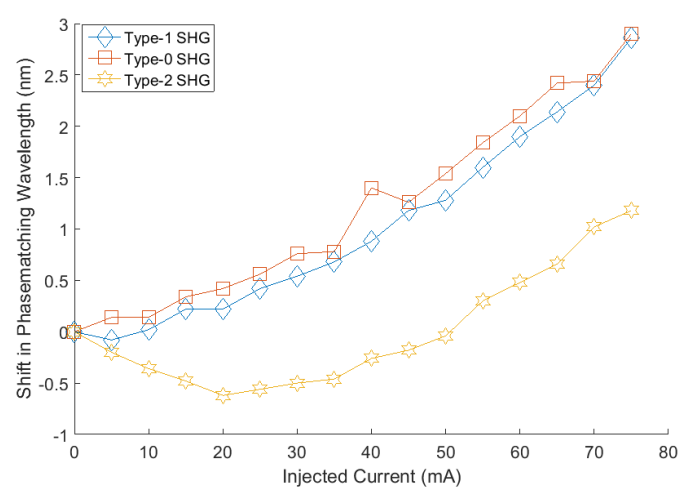

(c)

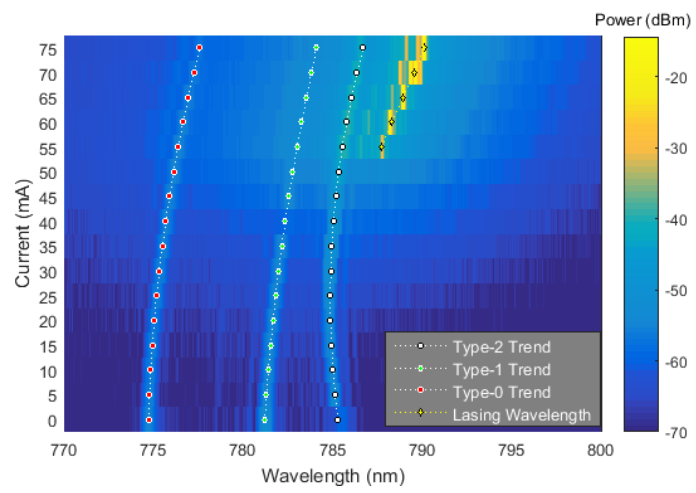

(b)

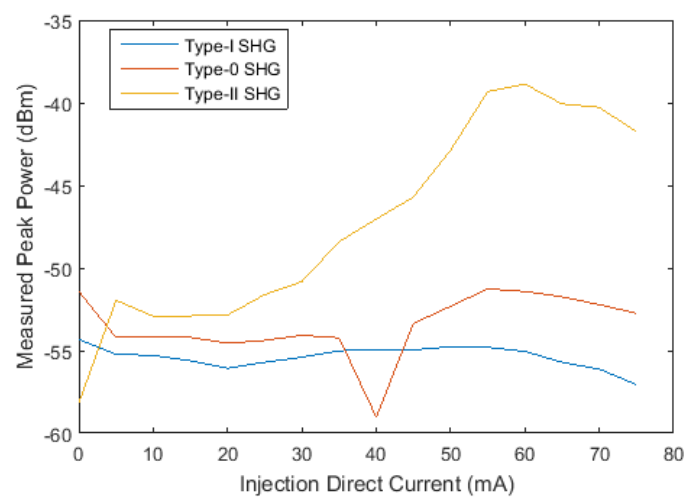

(d)

Figure 5.10: (a) Measured Spectra of the output from the laser diode sample injected with $50 \mathrm{~mW}$ Femtosecond pulse at different current. (b) A different representation to indicate the shift of the phasematching wavelength and the lasing wavelength. (c) The net shift of the phasematching wavelength for Type-1, Type-0 and Type-2. (d) The output power of each type of SHG . Power of Type-1 and Type-0 SHG remains relatively constant. The is a apparent gain behavior for the Type-2 SHG. Indicating the Bragg mode generated from the SHG is being amplified within the electrically active structure. 
The carrier only relationship with the phasematching position remains to be unknown. However, it can be extracted by applying an exponential model and rearranging Equation 5.4 and assuming the stage temperature remains stable.

$$
\begin{aligned}
\Delta \lambda_{e^{-} \_ \text {Type } 2}(I) & =\Delta \lambda_{\text {Type } 2}(I, T=0)-\frac{\Delta \lambda_{\text {Type } 2}}{\Delta T} *\left[\frac{\Delta T}{\Delta I} I+0\right] \\
& =C *(1-\exp (-B * I))
\end{aligned}
$$

where $\mathrm{C}$ and $\mathrm{B}$ are the coefficients of the exponential model. The result of the fitting is shown in Figure 5.11. Equation 5.4 can be rewritten as the following:

$$
\Delta \lambda_{\text {Type } 2}(I, T)=C *(1-\exp (-I / B))+\frac{\Delta \lambda_{T y p e 2}}{\Delta T} *\left[\frac{\Delta T}{\Delta I} I+T\right]
$$

In order to achieve self-pumping, given the $\lambda_{\text {laser }}\left(T_{0}\right)$ and $\lambda_{\text {Type2 }}\left(T_{0}\right)$, the following equation must be satisfied.

$$
\lambda_{\text {laser }}\left(T_{0}\right)+\Delta \lambda_{\text {laser }}(I, T)=\lambda_{\text {Type } 2}\left(T_{0}\right)+\Delta \lambda_{\text {Type } 2}(I, T)
$$

By utilizing the Equation 5.7, assuming that the Type-2 Phasematching is $786 \mathrm{~nm}$ at $30^{\circ} \mathrm{C}$, and using the current $\frac{\Delta T}{\Delta I}$ coefficient for the unbonded sample, the condition where the lasing wavelength and the phasematching point is satisfied is shown in Figure 5.12b. A different $\frac{\Delta T}{\Delta I}$ is also used to simulate the situation when the sample is bonded. A bonded sample would have a better thermal conductance and electrical conductance as the sample is no longer just sit on the surface of the copper block. This increase in thermal conductance and reduction in resistance will reduce the thermal effect generated as a result of current injection. Therefore, the coefficient, $\frac{\Delta T}{\Delta I}$ should be reduced for the bonded sample. The reduction of the thermal effect induced by the current would make 


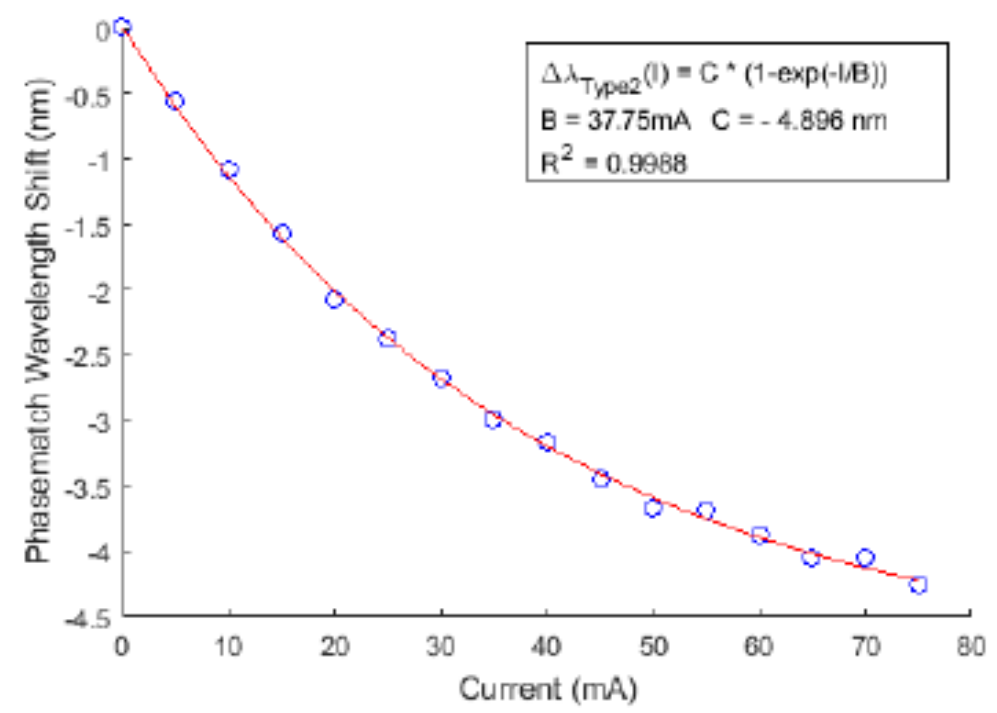

Figure 5.11: Exponential fit for the carrier effect uncoupled the thermal effect from the current injection. Strong indication of the contribution of the blue shift from the free carriers

the self-pumped condition less sensitive to the current as expected. This will reduce the amount of temperature degree that needed to be tuned in order to phasematch at different current level. From this simulation, it indicates that room-temperature selfpumped operation is highly feasible.

\subsection{Self-pumped Experiment}

To test the self-pumped process, the simplest experiment to perform is through SPDC. As SPDC does not require high power and can be easily detected with a temporal coincidence measurement. The experimental setup would have a bonded laser diode mounted on a copper block with TEC installed. A electrical probe is used to inject current into the p-contact pad while the copper block as the ground. The operation would preferably in DC mode. The laser output is coupled of the laser with a 40x objective lens and passed through a 1150nm long pass filter where the pump is filtered out. By following the solution outlined by Equation 5.7, the current and the temperature can be tuned 


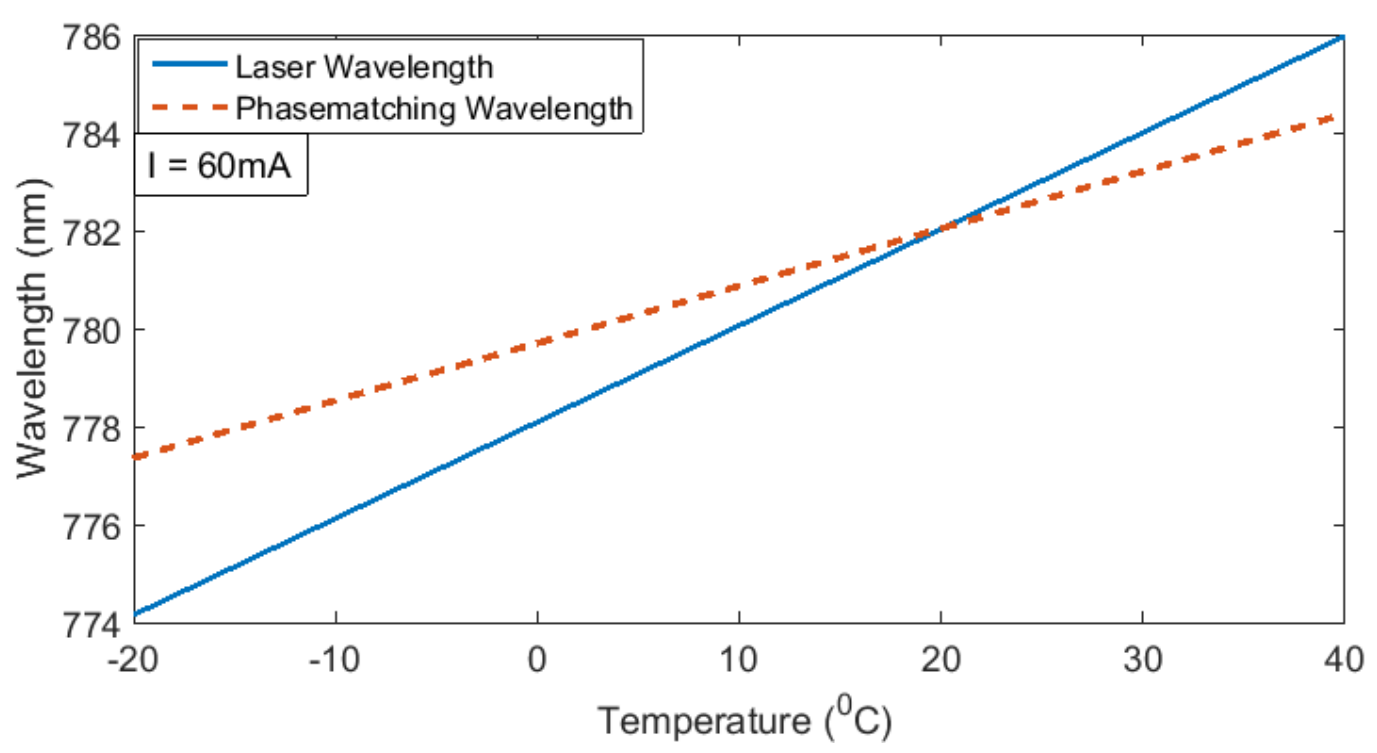

(a)

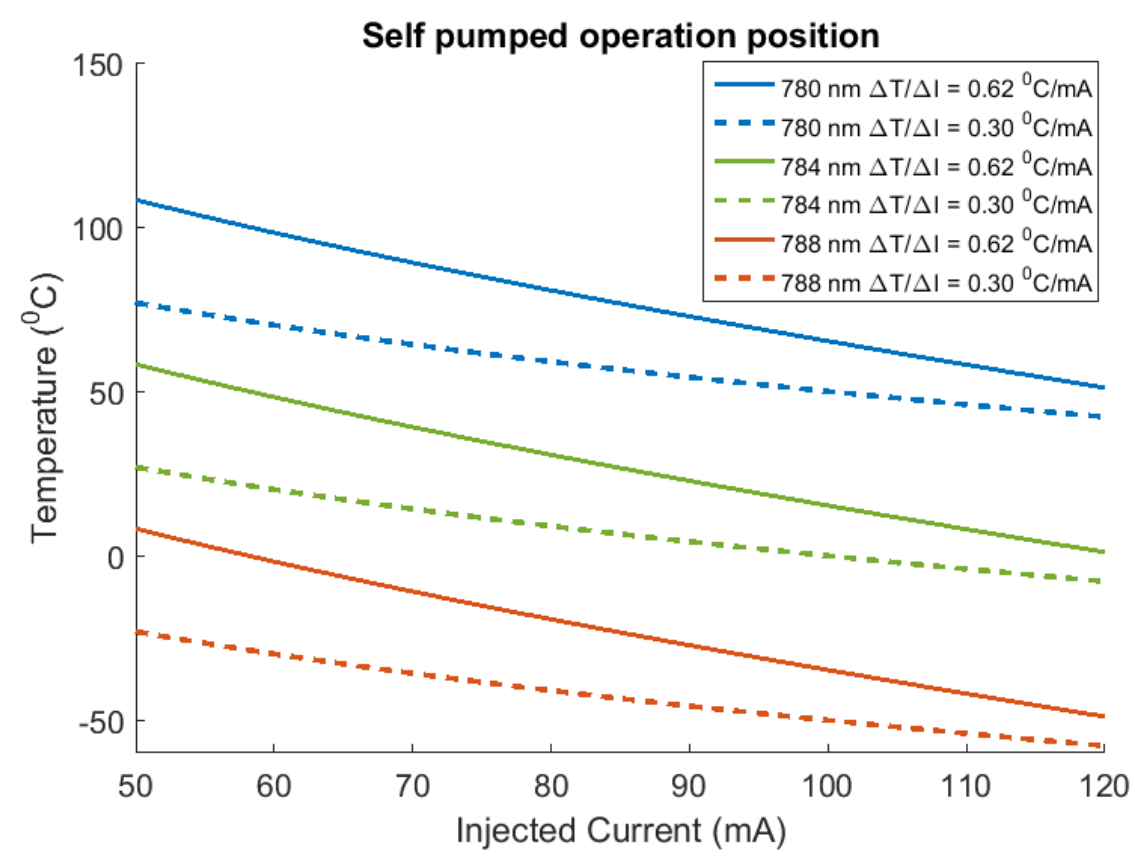

(b)

Figure 5.12: (a) Laser and phasematching wavelength from the Eq. 5.7 with lasing wavelength as $784 \mathrm{~nm}$ and $\frac{\Delta T}{\Delta I}$ as $0.3^{0} \mathrm{C} / \mathrm{mA}$. The intersection indicates the position where the self-pumping will occur (b) The line indicate the position where the lasing wavelength and the phasematching position coincides. The wavelength indicated at the legend is assumed to be the lasing wavelength at $30^{\circ} \mathrm{C}$ with $60 \mathrm{~mA}$ direct current. 
to the expected position to facilitate the self-pumping SPDC. The entangled photons generated from the self-pumped process would be coupled into a temporal coincidence measurement system, which is consisted of a free running single photon detector and a gated single photon detector. However, at the time of the completing this thesis, the fabrication process has not fully refined, leading to premature breakdown of the device due to contact issues, unstable output power, low power output. More time is needed to refine the fabrication process before the self-pumped process can be demonstrated.

\subsection{Summary of Parametric generation in BRL}

This chapter has laid out the foundation work towards characterizing and operating BRL structure in self-pumped operation. The basic structure of the BRL is described. Laser performance and nonlinear performance has been demonstrated. For the laser performance, the bonded Fabry Perot laser has a threshold current of around $44 \mathrm{~mA}$ and an external differential quantum efficiency of 0.056. As for the nonlinear performance, it has a SHG normalized conversion efficiency as $0.08 \% \mathrm{~W}^{-} 1 / \mathrm{cm}^{2}$. Although the laser quality is not yet refined, laser property parameters have been extracted to create a preliminary model for predict the behavior of the lasing wavelength and the phasematching wavelength in response to temperature and current. This model will serve as a map to the operation point where the self-pumping process can be facilitated. Additional design to the laser diode such as bandwidth enhancement through tapering can also be implemented to reduce the stringent requirement of matching the lasing wavelength and the phasematching wavelength. This platform has proven to be promising for self-pumping process, and will be able to demonstrate its potential once the fabrication has been refined. 


\section{Chapter 6}

\section{Conclusion and Future Outlook}

The work in this thesis focuses on an in-depth experimental study of optical parametric devices. AlGaAs is used as the material system for the optical parametric generation due to the monolithic integration potential with nonlinear element, pump laser and detectors. As a result, it is able to provide a compact, highly efficient, parametric device. In particular, the study focuses on the Bragg Reflection Waveguides as the design platform due to its capability to facilitate nonlinear interaction through exact modal phasematching. This exact modal phasematching is achieved by introducing Bragg mode and modifying the dispersion behavior. By achieving phasematching with highly nonlinear material, optical parametric generation can be utilized to perform wavelength conversion and parametric gain. By incorporating a quantum well structure within the structure, implementation of laser can be accomplished as well. This will by able to provide the ability for the platform to achieve self-pumping. Experimentally, it has been shown that, through external pumping source, the power that can be coupled into the waveguide is only around $12 \mathrm{~mW}$. This coupled power level is relatively low due to the higher order nature of the bragg mode. However, this limitation can be easily overcome through the use of self-pumped, where AlGaAs multi-quantum well laser can easily output at the order of $100 \mathrm{~mW}$. This thesis focuses on the experimental characterization of the BRW capability to perform 
nonlinear interaction and the empirical model for BRL to achieve self-pumping in the nonlinear device with active medium.

The acceptance bandwidth is desired to be enhanced due to the narrow operation regime. The narrow acceptance bandwidth will reduce the flexibility of application as the pump wavelength has to be accurate. While this accuracy may be easily achievable in external pumping, for self-pumping process, it may prove to be difficult. By increasing the pump acceptance bandwidth, the self-pumping requirement will be able to be more flexible. To achieve the enhancement, the dependence between the phasematching position and the ridge width is leveraged. By tapering the waveguide ridge width as a means to enhance the bandwidth, a variation of phasematching position can be implemented along the propagation. This technique does not require the growth of a new wafer. It simply relies on the waveguide structure. SHG characterization experiment is performed on the devices with different tapering degrees. The SHG accepting bandwidth for a straight waveguide, which is about $0.5 \mathrm{~nm}$, can be extended to $17 \mathrm{~nm}$ at the expense of conversion efficiency. DFG characterization experiment is also performed to measure the conversion bandwidth in response to the pump detuning. The result shows that the idler generation bandwidth is very similar to the SHG generation bandwidth. This signifies that the pump accepting bandwidth can be probed through SHG and DFG experiment. Thus, this demonstrates the capability of the tapering as a simple solution for bandwidth enhancement. Also for straight waveguide, the DFG experiment is performed with the pump at the degenerate wavelength to characterize the signal conversion bandwidth. It has been demonstrated to be around 50nm HWHM (100nm FWHM) with the idler conversion efficiency as around $-37 \mathrm{~dB}$. Although this conversion efficiency is lower than the fiber and PPLN waveguide, it has a flatband conversion of $100 \mathrm{~nm}$. If proper pulse source can be utilized, the conversion efficiency can be improved significantly.

In an effort to understand more of the nonlinear behavior in different operation regime, Nonlinear Schrolinger Equation Solver is used to simulate the nonlinear conversion in 
both continuous wave and pulse operation. The nonlinear conversion results are also demonstrated in the simulation for the continuous wave. It is indicative that there is likely not enough input power level for a gain level that is measurable in the continuous wave. It shows that around $10 \mathrm{~W}$ of power is needed to see an appreciable gain. By leveraging the usage of the pulse-pump, a high peak power can be leveraged to promote a strong nonlinear conversion. But in pulse operation, group velocity mismatch behavior has also demonstrated in this chapter and the walk-off introduced by the mismatch will limit the effective length of operation to be very narrow. For the case when pulse-pump with CW-signal is used, due to the low duty cycle of the pulse pump and the usage of continuous wave signal, the gain measurement is challenging as the average increase in signal power is negligible in comparison with the average input power. In the experiment, the CW operation shows the idler conversion, but it does not suggest a measurable gain. And in the pulse-CW operation, there is an increase in loss as a function of pulse pump power. This is likely induced from the free carrier generation from the femtosecond pulse. A temporal synchronized pulsed pump and signal system with a proper pulse width would likely be able to resolve this situation and demonstrate the gain from the simulation. Lastly, wavelength conversion is demonstrated on the BRW platform. When using the CW pump at the degenerate wavelength, a flat-band conversion with 100nm FWHM bandwidth and $-37 \mathrm{~dB}$ center at around 1560nm has been demonstrated. Also, when pumped in the non-degenerate wavelength, the conversion can be pushed to generate from 1650 to $2160 \mathrm{~nm}$ and it is only limited by the equipment. This shows the capability of BRW platform to perform NIR conversion.

Finally, a new generation of Bragg Reflection Waveguide Diode Laser is experimentally characterized for its laser performance and nonlinear conversion efficiency in an effort to achieve self-pumping. It has demonstrated a $2 \mathrm{~mW}$ output in DC operation, and a $8 \mathrm{~mW}$ output in pulse operation. It has a threshold current of $44 \mathrm{~mA}$ and external differential quantum efficiency of 0.058 . When operating in DC mode with the unbonded 
sample, the lasing wavelength is around $788 \mathrm{~nm}$. The BRL device has a SHG normalized conversion efficiency equals to $0.087 \% \mathrm{~W}^{-1} \mathrm{~cm}^{-2}$. Overall, the low nonlinear conversion efficiency and the low output power can be attributed to the fact that the fabrication has yet to be finalized in terms of quality. Even though the laser device quality is sub-par, device parameters, such as phasematching and lasing wavelength in response to current and temperature, are extracted to develop a model for locating the self-pumping operation point. This model demonstrates that this is a high possibility that there will be a room-temperature operation point where a self-pumped nonlinear process can be facilitated.

To conclude, this thesis demonstrated the capability of generation from telecom band to $2 \mathrm{um}$, the feasibility of self-pumping, the enhancement of the acceptance bandwidth, and the simulation to achieve optical amplification. This work pushes forward another step to promote BRW platform as a highly scalable, efficient optical parametric devices.

\subsection{Future Work}

This thesis has demonstrated the effect of tapering on the BRW structure. It also investigates into the regime where the device can work as an optical amplifier and wavelength conversion. In addition, it has built up a model to locate the self-pumping operation point for the laser diode.

The lower conversion efficiency can be mainly attributed to the fact that free space coupling into the Bragg mode is highly inefficient due to the high-order mode. Nonetheless, the platform has demonstrated the capability for wavelength conversion and the improvement in bandwidth. This challenge can be easily resolved with a development of the BRL where the lasing mode is in Bragg mode. The current laser device has yet to be refined in terms of fabrication quality, leading to the inability for a long duration of DC mode operation. This limitation is the only factor preventing the device to prove 
its self-pumping capability. Once it is refined, the laser device will have a high possibility of achieving self-pumping with more CW power within the cavity than the external injection will able to provide.

Moreover, there may be a difficulty in aligning the lasing wavelength and phasematching wavelength. However, the bandwidth enhancement techniques demonstrated in this thesis can be utilized to improve the accepting bandwidth. This will lead to a much more robust design as the design will be less sensitive to the lasing wavelength fluctuation as a result of fabrication. As a result, the design will have the ease of performing nonlinear interaction for wavelength conversion, optical parametric gain.

Lastly, once the laser has proven to function as a self-pumped parametric device, more sophisticated laser design can be developed to improve the generated laser power within the cavity. This will further improve the nonlinear conversion efficiency of BRW platform and provide a novel self-pumped, highly efficiency, compact and monolithically-integrated optical parametric device. 


\section{Bibliography}

[1] J. M. H. Elmirghani and H. T. Mouftah. All-optical wavelength conversion: technologies and applications in dwdm networks. IEEE Communications Magazine, 38(3):86-92, Mar 2000.

[2] MH Chou, I Brener, MM Fejer, EE Chaban, and SB Christman. 1.5-m-band wavelength conversion based on cascaded second-order nonlinearity in linbo waveguides. IEEE Photon. Technol. Lett, 11(6):653-655, 1999.

[3] Dongpeng Kang, Arthur Pang, Yuxiang Zhao, and Amr S. Helmy. Two-photon quantum state engineering in nonlinear photonic nanowires. J. Opt. Soc. Am. B, 31(7):1581-1589, Jul 2014.

[4] D Rehle, D Leleux, M Erdelyi, F Tittel, M Fraser, and S Friedfeld. Ambient formaldehyde detection with a laser spectrometer based on difference-frequency generation in ppln. Applied Physics B, 72(8):947-952, 2001.

[5] K. E. Whittaker, L. Ciaffoni, G. Hancock, R. Peverall, and G. A. D. Ritchie. A dfg-based cavity ring-down spectrometer for trace gas sensing in the mid-infrared. Applied Physics B, 109(2):333-343, 2012.

[6] Robert W Boyd. Nonlinear optics. Academic press, 2003.

[7] Zhi Tong, Carl Lundström, PA Andrekson, CJ McKinstrie, Magnus Karlsson, DJ Blessing, Ekawit Tipsuwannakul, BJ Puttnam, Hiroyuki Toda, and L Grüner- 
Nielsen. Towards ultrasensitive optical links enabled by low-noise phase-sensitive amplifiers. Nature Photonics, 5(7):430-436, 2011.

[8] Karol Krzempek, Grzegorz Sobon, and Krzysztof M Abramski. Dfg-based mid-ir generation using a compact dual-wavelength all-fiber amplifier for laser spectroscopy applications. Optics express, 21(17):20023-20031, 2013.

[9] Ulrike Willer, Mohammad Saraji, Alireza Khorsandi, Peter Geiser, and Wolfgang Schade. Near-and mid-infrared laser monitoring of industrial processes, environment and security applications. Optics and lasers in engineering, 44(7):699-710, 2006.

[10] Majid Ebrahim-Zadeh and Irina T Sorokina. Mid-infrared coherent sources and applications. Springer Science \& Business Media, 2008.

[11] Frank K Tittel, Dirk Richter, and Alan Fried. Mid-infrared laser applications in spectroscopy. In Solid-State Mid-Infrared Laser Sources, pages 458-529. Springer, 2003.

[12] Peter Werle, Franz Slemr, Karl Maurer, Robert Kormann, Robert Mücke, and Bernd Jänker. Near-and mid-infrared laser-optical sensors for gas analysis. Optics and lasers in engineering, 37(2):101-114, 2002.

[13] Yoram J Kaufman and Lorraine A Remer. Detection of forests using mid-ir reflectance: an application for aerosol studies. IEEE Transactions on Geoscience and Remote Sensing, 32(3):672-683, 1994.

[14] Vincenzo Spagnolo, Pietro Patimisco, Simone Borri, Gaetano Scamarcio, Bruce E Bernacki, and Jason Kriesel. Part-per-trillion level sf 6 detection using a quartz enhanced photoacoustic spectroscopy-based sensor with single-mode fiber-coupled quantum cascade laser excitation. Optics letters, 37(21):4461-4463, 2012. 
[15] Jerome Faist, Federico Capasso, Deborah L Sivco, Carlo Sirtori, Albert L Hutchinson, Alfred Y Cho, et al. Quantum cascade laser. Science, 264(5158):553-556, 1994.

[16] Yu Yao, Anthony J Hoffman, and Claire F Gmachl. Mid-infrared quantum cascade lasers. Nature Photonics, 6(7):432-439, 2012.

[17] G Wysocki, R Lewicki, RF Curl, FK Tittel, L Diehl, F Capasso, M Troccoli, G Hofler, D Bour, S Corzine, et al. Widely tunable mode-hop free external cavity quantum cascade lasers for high resolution spectroscopy and chemical sensing. Applied Physics B, 92(3):305-311, 2008.

[18] M Razeghi, B Gökden, S Tsao, A Haddadi, N Bandyopadhyay, and S Slivken. Widely tunable single-mode high power quantum cascade lasers. In SPIE Microtechnologies, pages 806905-806905. International Society for Optics and Photonics, 2011.

[19] Benedikt Schwarz, Peter Reininger, Hermann Detz, Tobias Zederbauer, Aaron Maxwell Andrews, Werner Schrenk, and Gottfried Strasser. Monolithically integrated mid-infrared quantum cascade laser and detector. Sensors, 13(2):2196$2205,2013$.

[20] Y Bai, N Bandyopadhyay, Slivken Tsao, S Slivken, and M Razeghi. Room temperature quantum cascade lasers with $27 \%$ wall plug efficiency. Applied Physics Letters, 98(18):1102, 2011.

[21] I Vurgaftman and JR Meyer. Analysis of limitations to wallplug efficiency and output power for quantum cascade lasers. Journal of applied physics, 99(12):123108, 2006.

[22] A Vicet, DA Yarekha, A Perona, Y Rouillard, S Gaillard, and AN Baranov. Trace gas detection with antimonide-based quantum-well diode lasers. Spectrochimica Acta Part A: Molecular and Biomolecular Spectroscopy, 58(11):2405-2412, 2002. 
[23] JEFF HECHT. Photonic frontiers: Antimonide lasers fill hole in the mid-infrared spectrum. Development, 1:05, 2010.

[24] WW Bewley, JR Lindle, CS Kim, M Kim, CL Canedy, I Vurgaftman, and JR Meyer. Lifetimes and auger coefficients in type-ii w interband cascade lasers. Applied Physics Letters, 93(4):041118, 2008.

[25] William W Bewley, Chadwick L Canedy, Chul Soo Kim, Mijin Kim, Charles D Merritt, Joshua Abell, Igor Vurgaftman, and Jerry R Meyer. Continuous-wave interband cascade lasers operating above room temperature at $\lambda=4.7-5.6 \mu \mathrm{m}$. Optics express, 20(3):3235-3240, 2012.

[26] I Vurgaftman, R Weih, M Kamp, JR Meyer, CL Canedy, CS Kim, M Kim, WW Bewley, CD Merritt, J Abell, et al. Interband cascade lasers. Journal of Physics D: Applied Physics, 48(12):123001, 2015.

[27] CS Kim, M Kim, J Abell, WW Bewley, CD Merritt, CL Canedy, I Vurgaftman, and JR Meyer. Mid-infrared distributed-feedback interband cascade lasers with continuous-wave single-mode emission to 80 c. Applied Physics Letters, 101(6):061104, 2012.

[28] Yuchao Jiang, Lu Li, Zhaobing Tian, Hao Ye, Lihua Zhao, Rui Q Yang, Tetsuya D Mishima, Michael B Santos, Matthew B Johnson, and Kamjou Mansour. Electrically widely tunable interband cascade lasers. Journal of Applied Physics, 115(11):113101, 2014.

[29] D Boucher, W Chen, J Burie, and P Peze. A novel cw optical laser-based differencefrequency infrared spectrometer. In Annales de Physique, volume 23, pages 247-248. EDP SCIENCES 7, AVE DU HOGGAR, PARC D ACTIVITES COURTABOEUF, BP 112, F-91944 LES ULIS CEDEXA, FRANCE, 1998. 
[30] Sanja Zlatanovic, Jung S Park, Slaven Moro, Jose M Chavez Boggio, Ivan B Divliansky, Nikola Alic, Shayan Mookherjea, and Stojan Radic. Mid-infrared wavelength conversion in silicon waveguides using ultracompact telecom-band-derived pump source. Nature Photonics, 4(8):561-564, 2010.

[31] Richard Soref. Mid-infrared photonics in silicon and germanium. Nature Photonics, $4(8): 495-497,2010$.

[32] Joseph C Palais. Fiber optic communications. Prentice Hall Englewood Cliffs, 1988.

[33] W Sohler, W Grundkötter, H Herrmann, JH Lee, YH Min, V Quiring, H Suche, R Schiek, T Pertsch, F Lederer, et al. All-optical wavelength conversion, parametric amplification, multiplexing, and switching in integrated ppln-devices. In 2006 International Conference on Transparent Optical Networks, volume 2, pages 185-188. IEEE, 2006.

[34] Emmanuel Desurvire. Erbium-doped fiber amplifiers: principles and applications. Wiley-Interscience, 2002.

[35] Michael J Connelly. Semiconductor optical amplifiers. Springer Science \& Business Media, 2007.

[36] Mohammed N Islam. Raman amplifiers for telecommunications. IEEE journal of selected topics in quantum electronics, 8(3):548-559, 2002.

[37] Zhi Tong and Stojan Radic. Low-noise optical amplification and signal processing in parametric devices. Adv. Opt. Photon., 5(3):318-384, Sep 2013.

[38] Jonas Hansryd and Peter A Andrekson. Broad-band continuous-wave-pumped fiber optical parametric amplifier with 49-db gain and wavelength-conversion efficiency. IEEE Photonics Technology Letters, 13(3):194-196, 2001. 
[39] Paul L Voss, Renyong Tang, and Prem Kumar. Measurement of the photon statistics and the noise figure of a fiber-optic parametric amplifier. Optics letters, 28(7):549$551,2003$.

[40] Renyong Tang, Paul L Voss, Jacob Lasri, Preetpaul Devgan, and Prem Kumar. Noise-figure limit of fiber-optical parametric amplifiers and wavelength converters: experimental investigation. Optics letters, 29(20):2372-2374, 2004.

[41] Charles A. Brackett. Dense wavelength division multiplexing networks: Principles and applications. IEEE Journal on Selected Areas in Communications, 8(6):948-964, 1990.

[42] Hui Zang, Jason P Jue, Biswanath Mukherjee, et al. A review of routing and wavelength assignment approaches for wavelength-routed optical wdm networks. Optical Networks Magazine, 1(1):47-60, 2000.

[43] Terji Durhuus, Benny Mikkelsen, Carsten Joergensen, S Lykke Danielsen, and Kristian E Stubkjaer. All-optical wavelength conversion by semiconductor optical amplifiers. Journal of Lightwave Technology, 14(6):942-954, 1996.

[44] SJ Ben Yoo. Wavelength conversion technologies for wdm network applications. Journal of Lightwave Technology, 14(6):955-966, 1996.

[45] Tomoyuki Akiyama, Haruhiko Kuwatsuka, Nobuaki Hatori, Yoshiaki Nakata, Hiroji Ebe, and Mitsuru Sugawara. Symmetric highly efficient (/spl sim/0 db) wavelength conversion based on four-wave mixing in quantum dot optical amplifiers. IEEE Photonics Technology Letters, 14(8):1139-1141, 2002.

[46] J Yamawaku, H Takara, T Ohara, K Sato, A Takada, T Morioka, O Tadanaga, H Miyazawa, and M Asobe. Simultaneous 25 ghz-spaced dwdm wavelength conversion of 1.03 tbit/s $(103 \times 10 \mathrm{gbit} / \mathrm{s})$ signals in ppln waveguide. Electronics Letters, $39(15): 1,2003$ 
[47] Ying-Hao Kuo, Haisheng Rong, Vanessa Sih, Shengbo Xu, Mario Paniccia, and Oded Cohen. Demonstration of wavelength conversion at $40 \mathrm{gb} / \mathrm{s}$ data rate in silicon waveguides. Optics Express, 14(24):11721-11726, 2006.

[48] J. A. Giordmaine and Robert C. Miller. Tunable coherent parametric oscillation in linbo $_{3}$ at optical frequencies. Phys. Rev. Lett., 14:973-976, Jun 1965.

[49] Juan Ariel Levenson, Ph Grangier, I Abram, and Th Rivera. Reduction of quantum noise in optical parametric amplification. JOSA B, 10(11):2233-2238, 1993.

[50] Konstantin L Vodopyanov, O Levi, PS Kuo, TJ Pinguet, JS Harris, MM Fejer, B Gerard, L Becouarn, and E Lallier. Optical parametric oscillation in quasi-phasematched gaas. Optics letters, 29(16):1912-1914, 2004.

[51] Thomas Torounidis and Peter Andrekson. Broadband single-pumped fiber-optic parametric amplifiers. IEEE Photonics Technology Letters, 19(9):650-652, 2007.

[52] Kyo Inoue. Polarization independent wavelength conversion using fiber four-wave mixing with two orthogonal pump lights of different frequencies. Journal of lightwave technology, 12(11):1916-1920, 1994.

[53] Philip Russell. Photonic crystal fibers. science, 299(5605):358-362, 2003.

[54] KK Chow, C Shu, Chinlon Lin, and A Bjarklev. Polarization-insensitive widely tunable wavelength converter based on four-wave mixing in a dispersion-flattened nonlinear photonic crystal fiber. IEEE photonics technology letters, 17(3):624-626, 2005.

[55] Haisheng Rong, Ying-Hao Kuo, Ansheng Liu, Mario Paniccia, and Oded Cohen. High efficiency wavelength conversion of $10 \mathrm{gb} / \mathrm{s}$ data in silicon waveguides. Optics Express, 14(3):1182-1188, 2006. 
[56] Mark A Foster, Amy C Turner, Reza Salem, Michal Lipson, and Alexander L Gaeta. Broad-band continuous-wave parametric wavelength conversion in silicon nanowaveguides. Optics Express, 15(20):12949-12958, 2007.

[57] Thomas Richter, Rahman Nouroozi, Hubertus Suche, Wolfgang Sohler, and Colja Schubert. Ppln-waveguide based tunable wavelength conversion of qam data within the c-band. IEEE Photonics Technology Letters, 21(25):2085-2088, 2013.

[58] Takeshi Umeki, Masaki Asobe, and Hirokazu Takenouchi. In-line phase sensitive amplifier based on ppln waveguides. Optics express, 21(10):12077-12084, 2013.

[59] Joris Van Campenhout, Liu Liu, Pedro Rojo Romeo, Dries Van Thourhout, Christian Seassal, Philippe Regreny, Lea Di Cioccio, Jean-Marc Fedeli, and Roel Baets. A compact soi-integrated multiwavelength laser source based on cascaded inp microdisks. IEEE Photonics Technology Letters, 20(16):1345-1347, 2008.

[60] Mitsuhiro Matsumoto, Kazuaki Sasaki, Masaki Kondo, Takashi Ishizumi, Tadashi Takeoka, Hiroshi Nakatsu, Masanori Watanabe, Osamu Yamamoto, and Saburo Yamamoto. High-power $780 \mathrm{~nm}$ algaas narrow-stripe window structure lasers with window grown on facets. Japanese journal of applied physics, 32(5A):L665, 1993.

[61] Tao Li, Shuang-yan Deng, Xue Li, Xiu-mei Shao, Heng-jing Tang, and Hai-mei Gong. Responsivity performance of extended wavelength ingaas shortwave infrared detector arrays. In SPIE Sensing Technology+ Applications, pages 91000U-91000U. International Society for Optics and Photonics, 2014.

[62] Payam Abolghasem, Jun-Bo Han, Dongpeng Kang, Bhavin J Bijlani, and Amr S Helmy. Monolithic photonics using second-order optical nonlinearities in multilayercore bragg reflection waveguides. IEEE Journal of Selected Topics in Quantum Electronics, 18(2):812-825, 2012. 
[63] Payam Abolghasem. Phase-Matching Second-Order Optical Nonlinear Interactions using Bragg Reflection WAveguides: A Platform for Integrated Parametric Devices. PhD thesis, University of Toronto, 2011.

[64] Bhavin J Bijlani, Payam Abolghasem, and Amr S Helmy. Semiconductor optical parametric generators in isotropic semiconductor diode lasers. Applied Physics Letters, 103(9):091103, 2013.

[65] Mohammed N Islam and O Boyraz. Fiber parametric amplifiers for wavelength band conversion. IEEE Journal of selected topics in quantum electronics, 8(3):527-537, 2002.

[66] Dongpeng Kang. On-chip generation of quantum states of photons. PhD thesis, University of Toronto, 2015.

[67] Thomas Feuchter and Carsten Thirstrup. High precision planar waveguide propagation loss measurement technique using a fabry-perot cavity. IEEE photonics technology letters, 6(10):1244-1247, 1994.

[68] JG Mendoza-Alvarez, FD Nunes, and NB Patel. Refractive index dependence on free carriers for gaas. Journal of Applied Physics, 51(8):4365-4367, 1980. 